\title{
The Role of Unconventional Magnetism in the Insulator-Metal Transition of Perovskite Cobaltites
}

\author{
Juan Yu \\ Lanzhou, China
}

B.S., Lanzhou University, 2002

M.S., Lanzhou University, 2005

A Dissertation presented to the Graduate Faculty of the University of Virginia in Candidacy for the Degree of

Doctor of Philosophy

Department of Physics

University of Virginia

May, 2011

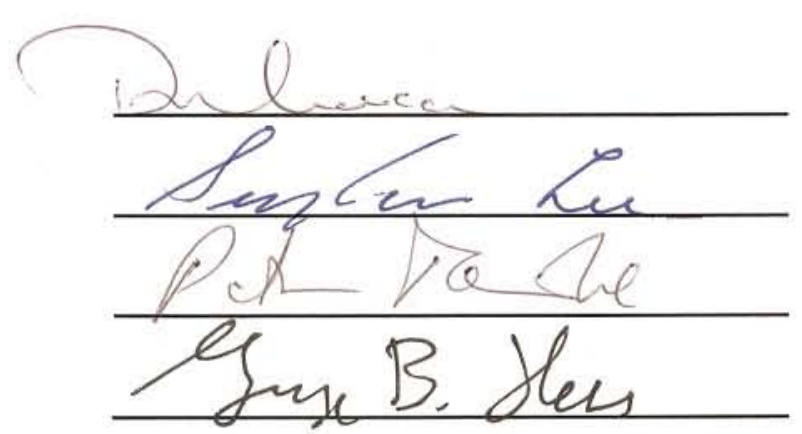




\begin{abstract}
In the perovskite cobaltite $\mathrm{LaCoO}_{3}$, the magnetic interactions resulting from the three possible spin states of the $\mathrm{Co}^{3+}$ ion $(\mathrm{S}=0,1$ and 2$)$ are sensitively affected by the lattice. Doping of charge carrier into this Mott insulator leads to two different effects: a) the lattice adjusts to accommodate the charges and b) different magnetic exchange may occur, which ultimately have consequences on the magnetism and electrical conductance. Thus, understanding the unconventional magnetism exhibited in this system and the insulator-metal transition that appears in the doped compounds is a challenge due to the complexity caused by the spin state, spin correlations and the magnetic exchange associated with $\mathrm{Co}^{3+}$ ions.

In this dissertation, the magnetic and lattice effects in doped $\mathrm{ACoO}_{3}(\mathrm{~A}=\mathrm{La}, \mathrm{Pr})$ are investigated on an atomic scale by neutron scattering techniques. In the hole-doped compounds, $\mathrm{La}_{1-\mathrm{x}} \mathrm{A}_{\mathrm{x}} \mathrm{CoO}_{3}(\mathrm{~A}=\mathrm{Ca}, \mathrm{Sr}, \mathrm{Ba})$, the magnetic phase separation (ferromagnetic and incommensurate or antiferromagnetic clusters of nanometer scale) and its trend are characterized. It is found that this trend is strongly correlated with the A-site dopant. A hypothesis is proposed to explain how the magnetic phase separation is driven by the lattice inhomogeneity and how the insulator-metal transition comes about.
\end{abstract}

In $\mathrm{LaCo}_{1-y} \mathrm{Ni}_{\mathrm{y}} \mathrm{O}_{3}$, an enhanced compression of the oxygen octahedron along the trigonal axis is invoked to break the degeneracy of the magnetic $\mathrm{Co}^{3+}$ without the local lattice breaking the trigonal symmetry. The magnetic correlation is found to be exclusively ferromagnetic in the spin glass state, and it is related to a magnetic excitation at $\sim 1.1 \mathrm{meV}$ down to $\mathrm{T} \rightarrow 0 \mathrm{~K}$ with a trace amount of doping. The nature of this excitation is argued to be associated with an indirect magnetic exchange. Two 
possibilities are discussed: a) a spin-flip excitation associated with a super-exchange ferromagnetic coupling via $\mathrm{Co}^{3+}-\mathrm{O}^{2-}-\mathrm{Ni}^{3+}$. The coupling can be confined in one or two dimensions resulting in a high probability of charge localization; b) a weak ferromagnetic coupling mediated by $\mathrm{Ni}^{3+}$ ions spins via $\mathrm{Co}^{3+}-e^{-}-\mathrm{Co}^{3+}$ within the localization range. Lastly, in $\mathrm{PrCoO}_{3}$, the atomic structure and neutron low energy spectra suggest that the thermal spin state transition may occur at a temperature above $300 \mathrm{~K}$, which is much higher than what was previously observed in $\mathrm{LaCoO}_{3}$.

To conclude, this thesis provides a microscopic approach to understanding the spinlattice interactions in this strongly correlated electron system that may be of broad interest to the field. 
Jo the years of A.D. 2005 to 2011. 


\section{Acknowledgements}

First, I would like to thank my advisor, Professor Despina Louca. She is not only the advisor for my research but also sometimes an advisor and/or good friend for the daily life. Our interactions in the past four years will have a profound influence on me in future. Next, I want to thank Dr. Daniel Phelan for helping me with the neutron scattering experiments; Prof. Kazuyoshi Yamada and Dr. Kazumasa Horigane for teaching me how to grow single crystals; and Dr. Kazuya Kamazawa and Dr. Keeseong Park for helping me with the bulk property measurements on Physical Property Measurement Systems.

Finally, I want to thank my mother. 感谢妈妈从始至终, 从无条件的全力支持。 感谢她陪伴我走过人生中每一个重要的脚步。 


\section{Contents}

List of Figures viii

List of Tables $\quad$ xiv

1 Introduction $\quad 1$

1.1 Introduction . . . . . . . . . . . . . . . . . . . 1

1.2 Layout of the Dissertation . . . . . . . . . . . . . . 8

2 Background of Perovskite Cobaltites 10

2.1 Crystal Structure . . . . . . . . . . . . . . . . . . . 10

2.2 Thermal Driven Spin-State Transition . . . . . . . . . . . . . . 16

2.3 Hole Doping Effects and Incommensurate Magnetic Phase in $\mathrm{La}_{1-x} \mathrm{Sr}_{x} \mathrm{CoO}_{3} 23$

2.4 Hole-Doping Induced Magnetic $\mathrm{Co}^{3+}$ Ion in the IS State . . . . . . . 24

3 Neutron Scattering $\quad 30$

3.1 Introduction . . . . . . . . . . . . . . . . . 30

3.2 Neutron Scattering Theory Highlights . . . . . . . . . . . . . . . . 33

3.3 Neutron Scattering Instruments . . . . . . . . . . . . . . . 40

4 Sample Preparation $\quad 43$

4.1 Powder Sample . . . . . . . . . . . . . . . . . . . . 43

4.2 Sample Quality Examination . . . . . . . . . . . . . . . . . . . . 44

4.3 Single Crystal Sample . . . . . . . . . . . . . . . . 45

5 Magnetic Phase Separation and Its Trend in $\operatorname{La}_{1-x} \mathrm{~A}_{x} \mathrm{CoO}_{3}(\mathrm{~A}=$ $\mathrm{Ca}^{2+}, \mathrm{Sr}^{2+}$ or $\left.\mathrm{Ba}^{2+}\right) \quad \mathbf{5 1}$

5.1 Global Magnetic State with Hole Doping . . . . . . . . . . . . . . . . 51

5.2 Coupled FM-IMT and the Magnetic Interaction Mechanism . . . . . 54

5.3 Magnetic Phase Separation Trends with A-site Dopant . . . . . . . . 57

5.4 A Hypothesis about Local Lattice Inhomogeneity Induced the Magnetic Phase Separation . . . . . . . . . . . . . . . 66 


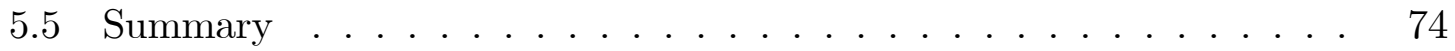

6 Unconventional Magnetism in the Insulating State of $\mathrm{LaCo}_{1-y} \mathrm{Ni}_{y} \mathrm{O}_{3} \mathbf{7 5}$

6.1 Effects of Doping Magnetic Ion $\mathrm{Ni}^{3+}$ and $\mathrm{Fe}^{3+}$ in $\mathrm{LaCoO}_{3}$. . . . . . 75

6.2 Spin States of $\mathrm{Co}^{3+}$ Ion in Trigonal Lattice . . . . . . . . . . . . . 78

6.3 Absence of AFM in Spin-Glass State . . . . . . . . . . . . . . . 90

6.4 Dilute Doped $\mathrm{LaCo}_{1-y} \mathrm{Ni}_{y} \mathrm{O}_{3}(y \leq 0.001) \ldots \ldots . \ldots . . \ldots . . \ldots 94$

6.4.1 Bulk Properties of Dilute Doped $\mathrm{LaCo}_{1-y} \mathrm{Ni}_{y} \mathrm{O}_{3}$. . . . . . . . 94

6.4.2 1.1 meV Excitation in Dilute Doped $\mathrm{LaCo}_{1-y} \mathrm{Ni}_{y} \mathrm{O}_{3}$. . . . . . 101

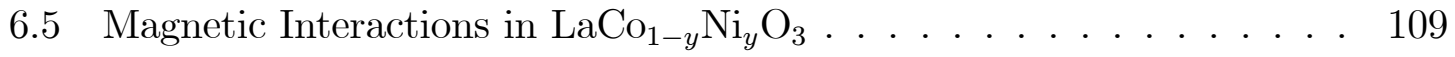

6.6 Summary . . . . . . . . . . . . . . . . 122

7 Spin Excitation in $\mathrm{PrCoO}_{3} \quad 123$

7.1 Introduction . . . . . . . . . . . . . . . . . . 123

7.2 When does Spin Excitation Occur in $\mathrm{PrCoO}_{3}$ ? . . . . . . . . . . . 126

7.2.1 Bulk magnetization- Onset temperature $\sim 200 \mathrm{~K}$. . . . . . 126

7.2.2 Crystal Structure- Absence of Distortion up to RT . . . . . . 128

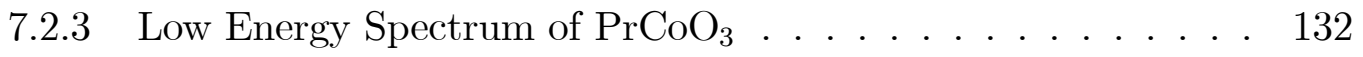

7.3 Summary . . . . . . . . . . . . . . . . . 134

8 Conclusions $\quad 136$

$\begin{array}{ll}\text { Bibliography } & 140\end{array}$ 


\section{List of Figures}

2.1 The perovskite structure and the $\mathrm{BO}_{6 / 2}$ octahedra . . . . . . . . . 11

2.2 The ideal cubic symmetry of the perovskite structure . . . . . . . . . 12

2.3 Rhombohedral unit cell and its two constituent psudocubic cells. . . . 14

2.4 Orthorhombic unit cell and its four constituent psudocubic cells. . . . 15

$2.5 d$ orbitals and the octahedron crystal field levels [18]. . . . . . . . . . 17

2.6 Electronic configurations of $\mathrm{Co}^{3+}$ ion at the LS, IS and HS state. . . . 18

2.7 Bulk electrical resistivity (upper panel) and magnetic susceptbility (lower panel) for a crystal of $\mathrm{LaCoO}_{3}$ as a function of temperature.

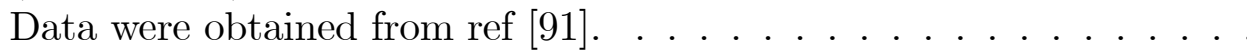

2.8 The low energy spectra of the powder sample $\mathrm{LaCoO}_{3}$ at different temperatures, after ref [61]. The following schematic shows the energy transfer of $0.6 \mathrm{meV}$ correspondes to the spin-flip transition within the $\mathrm{S}=1$ manifold.

2.9 Inelastic neutron scattering data obtained from the single crystals of $\mathrm{LaCoO}_{3}$ and $\mathrm{La}_{0.9} \mathrm{Sr}_{0.1} \mathrm{CoO}_{3}$ at SPINS at 55 and $100 \mathrm{~K}$. After ref [61]. 22

2.10 Magnetic phase diagram of $\mathrm{La}_{1-x} \mathrm{Sr}_{x} \mathrm{CoO}_{3}$ [49] . . . . . . . . . . . . .

2.11 The elastic neutron scattering contour map in the (hhl) plane for the single crystal of $\mathrm{La}_{0.85} \mathrm{Sr}_{0.15} \mathrm{CoO}_{3}$. The figure is from ref [62]. . . . .

2.12 The temperature dependence of the magnetic susceptibility of lightly Sr doped compounds $\mathrm{La}_{1-x} \mathrm{Sr}_{x} \mathrm{CoO}_{3}(x=0,0.002,0.005,0.010)$. After ref. [91].

2.13 The pair density functions (PDF) of $\mathrm{La}_{1-x} \mathrm{Sr}_{x} \mathrm{CoO}_{3}$ for $x=0.1,0.2$, 0.4 and 0.5 at $300 \mathrm{~K}$. After ref. [49]. . . . . . . . . . . .

2.14 The Jahn-Teller distortion for $\mathrm{Mn}^{3+}\left(3 d^{4}\right)$. The distortion prolongs the octahedron and breaks the degeneracy of the $t_{2 g}$ and $e_{g}$ orbitals. The

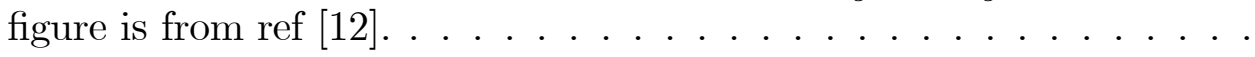

3.1 The photo of triple axis spectrometer SPINs at NCNR. . . . . . . . . 41

3.2 Schematic of time-of-flight instrument. . . . . . . . . . . . . . . . 42 
4.1 Schematic of a two-mirror image furnace, obtained from ref [22] . . . 46

4.2 Photo of the two-mirror image furnace . . . . . . . . . . . 46

4.3 Photo of grown single crystal for $\mathrm{LaCO}_{0.9} \mathrm{Ni}_{0.1} \mathrm{O}_{3} \ldots \ldots \ldots$. . . . . . 49

5.1 The magnetic phase diagrams for $\mathrm{Ca}$, Sr and Ba doping. The figure is from ref [44]. . . . . . . . . . . . . . . . . . . 52

5.2 Bulk magnetic susceptibilities for single crystals of $\mathrm{La}_{1-x} \mathrm{Ba}_{x} \mathrm{CoO}_{3}(x=0.03$ to 0.18 ) as a function of temperature. . . . . . . . . . 53

5.3 Double exchange between an IS $\mathrm{Co}^{3+}$ and a $\mathrm{LS} \mathrm{Co}{ }^{4+}$ ion. . . . . . . 55

5.4 AFM super-exchange between two IS $\mathrm{Co}^{3+}$ ions. . . . . . . . . . 56

5.5 The diagonal scans along [111] around the Bragg peak of (001) in single crystals of $\mathrm{La}_{0.95} \mathrm{Ca}_{0.05} \mathrm{CoO}_{3}$ and $\mathrm{La}_{0.9} \mathrm{Ca}_{0.1} \mathrm{CoO}_{3}$. No magnetic satellite peaks have been observed. . . . . . . . . . . . . . 58

5.6 The elastic neutron scattering contour in (hhl) plane around (001) position in the single crystal of $\mathrm{La}_{0.9} \mathrm{Ba}_{0.1} \mathrm{CoO}_{3}$ at $5 \mathrm{~K}$. The inset at the right upper corner is the contour for the $\mathrm{Sr}$ composition at the same doping concentration, $\mathrm{La}_{0.9} \mathrm{Sr}_{0.1} \mathrm{CoO}_{3}$. The magnetic super lattice scatterings appear more obvious in Ba sample than the ones in $\mathrm{Sr}$. .

5.7 (a)-(d): the diagonal scans below (black) and above (red) the magnetic transition temperature for $\mathrm{La}_{1-x} \mathrm{Ba}_{x} \mathrm{CoO}_{3}(x=0.18,0.15,0.10,0.03)$. (e)-(f) the compounds' corresponding order parameters, the FM (blue) and the satellite (green), compared with the bulk magnetic susceptibility, field-cool (black) and zero-field-cool (orange). The inset of (g) is the comparison of the integrated satellite intensity with $\chi_{\text {bulk }}$. . . .

5.8 Data for the Ba crystals as a function of hole concentration. (a) is a plot of the IC peak position, $\delta$, with $x \%$ which is compared to the dependence on $x \%$ obtained previously for Sr crystals. (b) is a plot of the order parameter for the two magnetic phases. (c) is a plot of the normalized satellite peak intensity, $I_{I C}$ and the (001) Bragg peak, $I_{F M}$. (d) is a plot of a comparison of the correlation lengths for the commensurate, $\xi_{F M}$ and incommensurate, $\xi_{I C}$. Note that $\xi$ is comparable in the two magnetic phases at all $x$, while the value of $80 \AA$ for the $18 \%$ is a lower bound since in the experimental setup of TOPAN, the peaks became resolution limited. . . . . . . . . .

5.9 The correlation length, $\xi$, as a function of $\left\langle\mathrm{r}_{A}\right\rangle$, determined for the two magnetic components in $\mathrm{Ca}, \mathrm{Sr}$ and $\mathrm{Ba}$ crystals of 5(6) and $10 \%$. At a constant hole concentration, $\xi_{F M C}$ is always larger in Ca than in $\mathrm{Sr}$ and $\mathrm{Ba}$ crystals. No second magnetic phase has been detected in Ca. At the same time, as $\left\langle\mathrm{r}_{A}>\right.$ increases, $\xi_{F M C}$ and $\xi_{I C}$ become comparable. The lines are guides to the eye. . . . . . . . . . 
5.10 The average tolerance factor, $\left\langle t>\right.$, and its variance, $\sigma^{2}$, as a function of the average A-site ionic size, $\left\langle r_{A}>\right.$, in $10 \%$ doped $\mathrm{Ca}, \mathrm{Sr}$ and $\mathrm{Ba}$ compositions. . . . . . . . . . . . . . . .

5.11 The corona model discribes three different local lattices in $\mathrm{La}_{1-x}(\mathrm{Sr} / \mathrm{Ba})_{x} \mathrm{CoO}_{3}$ : (1) "Dopant-rich-FM" region shown as a red zone. In this area, the large size of the dopant leads the lattice to be more cubic-like, and the FM coupling is established via the DE through $\mathrm{Co}^{4+}{ }_{-} \mathrm{O}-\mathrm{Co}^{3+}$. (2) AFM region, shown as the yellow corona, is the area connecting the region (1) and the background (3). For the background (blue), its lattice properties is most close to the pure compound, $\mathrm{LaCoO}_{3}$, maintaining a significant trigonal distortion. Thus, the local lattice of region (2) gradually transfers the difference on the local lattice between (1) and (3). The AFM coupling is established between two Co ions with identical spin configuration via super-exchange. The right panel gave a schematic of two-dimension lattices in each region, to demonstrate the possible differences on the local lattices. . . . . . . . . . . .

6.1 Bulk magnetization as a function of temperature with dc mode under 200 Oe field in $\mathrm{LaCo}_{1-y} \mathrm{Ni}_{y} \mathrm{O}_{3}$ and $\mathrm{LaCo}_{1-y} \mathrm{Fe}_{y} \mathrm{O}_{3}$. . . . . . . . . .

6.2 The octahedron rotation angle, $\omega$, and the rhombohedral unit cell angle, $\alpha$, as a function of doping concentrations at $\mathrm{RT}$ for $\mathrm{La}_{1-x} \mathrm{Ba}_{x} \mathrm{CoO}_{3}$ (panel (a) and (c)) and $\mathrm{LaCo}_{1-y} \mathrm{Ni}_{y} \mathrm{O}_{3}$ and $\mathrm{LaCo}_{1-y} \mathrm{Fe}_{y} \mathrm{O}_{3}$ (panel (b) and $(\mathrm{d})) . \ldots \ldots \ldots \ldots$

6.3 A schematic of the deformation. The black lines define one rhombohedral unit cell and the red lines indicate two constituent pseudocubic cells. The body diagonal [111] is shown as a blue line. A, B, C are three oxygen ions which lie in a plane normal to the [111]. $\mathrm{O}$ is the intersection point of this plane with the [111]. In an ideal cubic symmetry, oxygen ions are located in the mid point of the side of the cube, such as the position marked as $\mathrm{D}$. The rotation of $\mathrm{BO}_{6}$ octahedron around [111], $\omega$, thus corresponds to the angle $\angle$ AOD. For a given oxygen displacement value $x$, (i) shows as $\alpha>60^{\circ}$, (ii) $\alpha^{\sim} 60^{\circ}$ and (iii) $\alpha<60^{\circ}$. To maintain the regular shape of the octahedron, for a nontrivial oxygen displacement $x-(-0.25) \neq 0, \alpha$ has to be smaller than $60^{\circ}$. Otherwise, a compressed stress is applied along the trigonal axis shown with the orange arrows. . . . . . . . . . . . . . 
6.4 The octahedron strain $\varsigma$ as a function of $\omega$ for $\mathrm{LaCoO}_{3}(\# 1) ; \mathrm{La}_{1-x} \mathrm{Ba}_{x} \mathrm{CoO}_{3}$ (\#2 to 6 for $x=0.1$ to 0.5 ); $\mathrm{LaCo}_{1-y} \mathrm{Ni}_{y} \mathrm{O}_{3}$ (\#8 and 9 for $x=0.1$ and 0.4 ); and $\mathrm{LaCo}_{1-y} \mathrm{Fe}_{y} \mathrm{O}_{3}$ (\#10 and 11 for $y=0.1$ and 0.4 ). The black line is an empirical fitting for $\mathrm{Ba}$ doped samples. The inset at the upper corner is a function of $\varsigma$ in the space of $\alpha \geq 60^{\circ}$ and $\omega \geq 0^{\circ}$. It appears as a slanted curved plane with a lowest point at $\varsigma=0$ when $\alpha=60^{\circ}$ and $\omega=0^{\circ}$. The black dot is where $\mathrm{LaCoO}_{3}$ is located on this plane and the red lines are possible paths for the cases where $\alpha$ and $\omega$ change in a same manner. The inset at the lower corner is a blow up of the high $\omega$ region. The uncertainty is shown by error bars. . . . . .

6.5 (a) The PDF of $\mathrm{LaCo}_{0.6} \mathrm{Ni}_{0.4} \mathrm{O}_{3}$ at $\mathrm{T}=12,50,100,295 \mathrm{~K}$. (b) and (c) are the comparison of the first PDF peak between $\mathrm{LaCo}_{1-y} \mathrm{Ni}_{y} \mathrm{O}_{3}$ and $\mathrm{La}_{1-x} \mathrm{Ba}_{x} \mathrm{CoO}_{3}$ for $\mathrm{x}=\mathrm{y}=10 \%$ and $40 \%$ at $295 \mathrm{~K}$, respectively. . .

6.6 The neutron elastic scattering along the direction of $(h, h, 1-|h|)$ for $\mathrm{LaCo}_{0.9} \mathrm{Ni}_{0.1} \mathrm{O}_{3}$ single crystal at $\mathrm{T}=5$ and $80 \mathrm{~K}$. A broad intensity due to magnetic scattering at low temperature only appears around the (001) Bragg peak, indicating the presence of short-range ferromagnetic correlations. The inset shows the ferromagnetic order parameter compared with field-cooled bulk magnetization. (b) is an elastic contour map of the $(h h l)$ plane. . . . . . . . . . . . . . . . 92

6.7 (a) Temperature dependence of the magnetic susceptibility for $\mathrm{LaCo}_{1-y} \mathrm{~B}_{y} \mathrm{O}_{3}$ $(\mathrm{y}=0,0.005,0.01, \mathrm{~B}=\mathrm{Ni}$ and $\mathrm{Fe}$ ). (b) The field dependence of the magnetization for $\mathrm{LaCo}_{0.995} \mathrm{Ni}_{0.005} \mathrm{O}_{3}$ at $\mathrm{T}=4,10$ and $30 \mathrm{~K}$. The lines are the fitting with the modified Brillouin function. . . . . . . . .

6.8 Electric resistivity as a function of temperature on the single crystal $\mathrm{LaCo}_{0.995} \mathrm{Ni}_{0.005} \mathrm{O}_{3}$ and $\mathrm{LaCo}_{0.99} \mathrm{Ni}_{0.01} \mathrm{O}_{3}$. Each sample is also measured

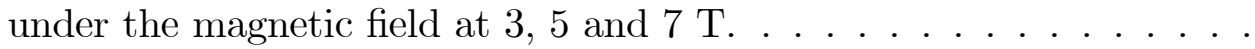

6.9 (a) The low energy spectra at $11 \mathrm{~K}$ among different compositions, $\mathrm{LaCo}_{0.995} \mathrm{Ni}_{0.005} \mathrm{O}_{3}, \mathrm{LaCo}_{0.995} \mathrm{Fe}_{0.005} \mathrm{O}_{3}$ and $\mathrm{LaCoO}_{3}$. (b) The spectra for $\mathrm{LaCO}_{1-y} \mathrm{Ni}_{y} \mathrm{O}_{3}$ at different doping concentrations, $y=0.005,0.01$ and 0.1. (c) The temperature dependence of the $1.1 \mathrm{meV}$ excitation. All data are symmetrized by the detail balance and normalized to the absolute units . . . . . . . . . . . . . . . . .

6.10 The low energy spectra under the field of $0,0.5,0.72$ and $2 \mathrm{~T}$ at $5 \mathrm{~K}$ on the $\mathrm{LaCo}_{0.99} \mathrm{Ni}_{0.01} \mathrm{O}_{3}$ powder sample at $5 \mathrm{~K}$ at the constant $\mathrm{Q}$ position of $(0,0,1)$. The inset of $(d)$ is the magnetic field dependence of the excitation, measured at the constant energy transfer of $1.2 \mathrm{meV}$ at 5 $\mathrm{K}$. The green lines are the fitting with three Gaussian functions and the red line is the total profile of the fitting which is one Gaussian in (a), three Gaussians in (b) and (c) and a constant background in (d). 
6.11 (a) and (b) are the low energy spectra on the single crystal $\mathrm{LaCo}_{0.995} \mathrm{Ni}_{0.005} \mathrm{O}_{3}$ obtained on SPINS. The energy scans were measured at the constant $\mathrm{Q}$ position of $(0,0,1.1)$ and $(0.5,0.5,0)$ at $5 \mathrm{~K}$ and $55 \mathrm{~K}$. (c) and (d) are the inelastic neutron contours obtained on MACS. They were measured at the constant energy transfer of $1.1 \mathrm{meV}$ in (hhl) plane at $1.5 \mathrm{~K}$ and $55 \mathrm{~K}$. The Q-dependence of inelastic intensities only appear around the Bragg peaks as a broad peak. The $(0,0,0)$ peak also contains the direct incident beam and its majority intensity is masked by beam stopper. . . . . . . . . . . . . . . . . .

6.12 Possible configurations for the FM coupling via super-exchange between $\mathrm{Ni}^{3+}$ and $\mathrm{Co}^{3+}$ ions. . . . . . . . . . . . . . . . . . . . . . . . 111

$6.13 e_{g}$ orbital orientations for several small FM clusters. The empty $e_{g}$ orbitals are drawn as red open and the occupied ones are blue solid. (a) shows the cases of two-metal-ions clusters; (a') corresponds to the case where the clusters are formed by four ions and for the recipient ion only one of the $e_{g}$ orbitals is empty. The cluster can develop along $z$ direction if the coupling empty orbital is $d_{z^{2}}$ (the first case in (a')) or the cluster can grow in $x-y$ plane if the empty one is $d_{x^{2}-y^{2}}$ (the last two cases in (a')). (b) shows the possbile orbital orientation in the seven-site FM cluster when the center recipient ion has both $e_{g}$ orbitals

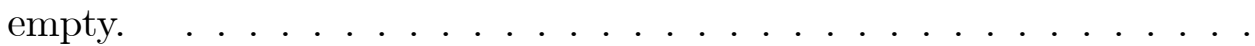

6.14 The configurations of small FM clusters for the 2-site up to 7-site models. For the 4-site model of a corner-shape in 3D and 5-site and 7-site models, the calculated magnetic intensities in (hhl) plane show peaks at the positions of $(h \pm 0.5, k \pm 0.5, l \pm 0.5)$. Therefore these models are ruled out. . . . . . . . . . . . . . . .

6.15 (a) and (b) are the cuts along the (hh1) and (00l) directions of the inelastic contour at $1.5 \mathrm{~K}$. The experiment data are shown as the open circles. The red solid line is the calculated peak based on the 2-site model, the blue lines and the black lines correspond to the 3 -site and 4-site models, respectively. (c) and (d) are the cuts at $100 \mathrm{~K}$, compared with the 2 -site model. . . . . . . . . . . . . . . . .

6.16 A cartoon of the Co lattice in two dimension. The blue open circles are the spin-zero $\mathrm{Co}^{3+}$ ions and the red solid circles are the magnetic ones. One $\mathrm{Ni}^{3+}$ dopant is drawn in yellow. The grey lines represent the track of the itinerate electron provided by $\mathrm{Ni}^{3+}$. Within the localization range this electron contributes to the conductivity and at the same time mediates the FM coupling among the magnetic $\mathrm{Co}^{3+}$. The scattering from the randomly distributed magnetic $\mathrm{Co}^{3+}$ on the other hand localized the electron. . . . . . . . . . . . . . . 
7.1 As the crystal symmetry lowers from cubic to orthorhombic, the octahedra become buckled to each other. . . . . . . . . . . . . . . . . . 124

7.2 Temperature dependence of the bulk magnetic susceptibility of $\mathrm{PrCoO}_{3}$ and $\mathrm{PrAlO}_{3}$ under the applied field of 1000 Oe. . . . . . . . . . . 127

7.3 Temperature dependence of the bulk magnetic susceptibility of $\mathrm{PrCoO}_{3}$ after subtracting from $\mathrm{PrAlO}_{3}$. The data is compared with $\chi(T)$ of $\mathrm{LaCoO}_{3} . \ldots \ldots \ldots \ldots \ldots$

7.4 A summary of the Rietveld refinement results of $\mathrm{PrCoO}_{3}$ from 12 to $350 \mathrm{~K}$. (a) the lattice parameters of orthorhombic unit cell, a, b and c. (b) the unit cell volume compared with the one of $\mathrm{LaCoO}_{3}$. (c) three Co-O bond lengths in an octahedron. (4) two Co-O-Co bond angles.

7.5 The $\mathrm{PDF}$ of $\mathrm{PrCoO}_{3}$ from 12 to $350 \mathrm{~K}$ obtained on the time-of-flight machine NPDF. The inset is the blow up of the first peak. . . . . . . 131

7.6 A comparison of the $\mathrm{PDF}$ of $\mathrm{PrCoO}_{3}$ at $12 \mathrm{~K}$ between the experiment data and the calculated model based on the orthorhombic crystal symmetry. . . . . . . . . . . . . . . .

7.7 Temperature dependence of the energy spectra of $\mathrm{PrCoO}_{3}$ powder from 10 to 350 K obtained on DCS. . . . . . . . . . . . . . . . . . . . . . . 134 


\section{List of Tables}

2.1 Atomic positions in cubic perovskite $\mathrm{ABO}_{3} \ldots \ldots$. . . . . . . . . 12

2.2 The lowered symmetries and the corresponding $\mathrm{BO}_{6 / 2}$ rotations . . . 13

2.3 Atomic positions in rhombohedral perovskite $\mathrm{ABO}_{3}$ based on rhombohedral setting. . . . . . . . . . . . . . . . . . . 15

2.4 Atomic positions in orthorhombic perovskite $\mathrm{ABO}_{3} \ldots \ldots \ldots$

5.1 A list of tolerance factors, t, evaluated only near the dopant rich regions for $\mathrm{Ca}, \mathrm{Sr}$ and $\mathrm{Ba}$ doping. The $<\mathrm{t}>$ are calculated from values obtained from the crystallographic refinement at room temperature for the samples with doping $\mathrm{x}=\mathrm{y}=0.1$. t-dopint-rich is evaluated considering only the immediate vicinity of the dopant; the percent increase is evaluated based on the comparison with the $t$ of $\mathrm{LaCoO}_{3}$. ICM refers to incommensurate ordering while $\mathrm{CM}$ refers to commensurate magnetic ordering. . . . . . . . . . . . . .

6.1 The Rietveld refinement results on $\mathrm{LaCo}_{1-y} \mathrm{~B}_{y} \mathrm{O}_{3}$ for $\mathrm{B}=\mathrm{Ni}$ and $\mathrm{Fe}$, $\mathrm{y}=0.1$ and 0.4 from $\mathrm{T}=12-295 \mathrm{~K}$. The listed parameters refer to space group R-3c (No. 167) in the rhombohedral unit cell. The La position $(2 \mathrm{a})$ is at $(1 / 4,1 / 4,1 / 4)$, the $\mathrm{Co} / \mathrm{Ni} / \mathrm{Fe}(2 \mathrm{~b})$ is at $(0,0,0)$ and the oxygen position $(6 e)$ is given by $(-x, 1 / 2+x, 3 / 4)$. Listed is the lattice constants and weighted R-factor. . . . . . . . . . . . .

6.2 A continued Table after table 4.1. The data of $\mathrm{Ni}$ and Fe doped systems are also included . . . . . . . . . . . . . . . . 93

6.3 The summary of the best fitted parameters for the magnetic depen-

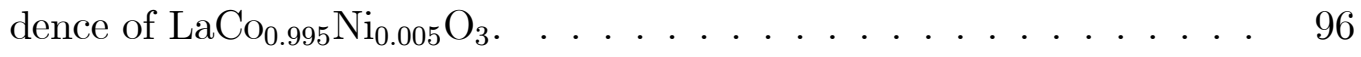

7.1 Rietveld Refinement Results for $\mathrm{PrCoO}_{3}$ from 12 to 350 K. . . . . . . 129 


\section{Chapter 1}

\section{Introduction}

\section{$1.1 \quad$ Introduction}

Similar to the way that many people are fascinated cooking, such as preparing delicious Kimchi (I assume you like it) out of dull plain cabbage by adding some magic spice, many solid-state physicists are fascinated by the concept of transforming electrically "dead" insulators into "live" conductors, a phenomenon known as an insulator-metal transition (IMT), which can often be brought about through the doping of charge carriers. This interest is driven by both practical applications and our intellectual curiosity about exactly how things work. For example, the microelectronics industry is founded upon using silicon chips, for which a semiconductor is doped with electrons or holes. Perhaps even more interesting is when holes are doped into cuprates, such as $\mathrm{La}_{2-x} \mathrm{Sr}_{x} \mathrm{CuO}_{4}$, for which high temperature superconductivity 
emerges [7].

Depending on the ground state of the parent compounds, doping can have essentially different effects [21]. In the above two examples, the first parent compound is a band insulator and the second one is a Mott insulator. In solid state physics, the fundamental problem is to find an adequate description for the behaviors of the outer shell electrons which determine the physical properties of the materials. This understanding becomes very complicated after the atoms are brought together into the form of a crystal, and so far two main theories lay the basis for two limiting situations. In one the outer electrons are treated to belong to the crystal as a whole, i.e., band theory [79]. In the other the outer electrons are assumed to be localized at specific atomic sites, i.e., the crystal field (CF) (or ligand-field) theory [9].

Band theory provides a conceptually simple explanation for how electrons move in solids. It predicts an insulating state when all bands are fully occupied or empty otherwise a metallic state occurs. Band insulators, therefore, are diamagnetic because the electrons are all paired so that there is no net magnetic moment. Though band theory has proven phenomenally successful on commercially important materials like silicon, it can not explain high temperature superconductivity, and it also gives a false prediction on the metallicity of many oxides such as $\mathrm{MnO}$ and $\mathrm{CoO}$.

The Mott insulating state was thus introduced to describe a situation where a material should be metallic according to band theory, but is insulating $[56,57]$. The insulating is due to the strong electron-electron repulsion energy, $\mathrm{U}$, which is stronger than 
the kinetic energy gain so that the electron cannot hop to the other atom. Because the electrons are localized in a Mott insulator, they may carry magnetic moments, and a magnetic order can be realized below the magnetic-ordering temperature. This implies that, unlike the band insulators, for the Mott insulator the internal degrees of freedom, such as spin and orbital, still survive [86], while at the same time there is a correlation between the magnetism and the IMT, which has always been a fascinating issue as exemplified in high Tc superconductivity of cuprates, $\mathrm{La}_{2-x} \mathrm{Sr}_{x} \mathrm{CuO}_{4}$ [7], and Colossal Magneto-resistivity (CMR) of manganites, $\mathrm{La}_{1-x} \mathrm{Ca}_{x} \mathrm{MnO}_{3}[75,88,48]$.

$\mathrm{LaCoO}_{3}$ is a Mott insulator and exhibits unusual magnetism and an IMT as its temperature is increased $[31,10,68,84,4,5, ?]$ (details in Chapter 2.2). These peculiar physical properties originate from the behavior of $\mathrm{Co}^{3+}$ ions' $3 d$ electrons and the covalent mixing with oxygen ions' $p$ orbitals. In classical crystal field theory, atomic $d$ orbitals are used, and the orbital degeneracies are lifted by the crystalline electrostatic field [89]. The magnetic moment of an ion is thus determined by the energy competition between the crystal field and the Hund's energy [12]. In the lattice accommodated by $\mathrm{LaCoO}_{3}$, these two energies happen to be comparable, so that the ground state of $\mathrm{Co}^{3+}$ with spin value $\mathrm{S}=0$, and two excited magnetic states with $\mathrm{S}=1$ and $\mathrm{S}=2$ are nearly degenerate in energy [11]. The electronic configuration of $d$ electrons of $\mathrm{Co}^{3+}$ in the $\mathrm{CF}$ not only determines its magnetic state but also affects its carrier mobility. By warming up to $~ 100 \mathrm{~K}$, a spin-state transition from the nonmagnetic ground state to magnetic states first occurs, and as temperature 
continues to rise above $\sim 500 \mathrm{~K}$ an IMT occurs resulting from a transformation of a localized to an itinerate $d$ electrons. [68].

The physical properties of $\mathrm{LaCoO}_{3}$ are complex both because the $d$ electrons of $\mathrm{Co}^{3+}$ ions determine the spin states for which the LS, IS and HS state can simultaneously be present and because, at the same time, $d$ electrons also participate in the conductivity, and in the pure compound there is a IMT with temperature. For doped $\mathrm{LaCoO}_{3}$, early studies based on the bulk properties showed that the magnetic and transport properties can be dramatically changed by both hole doping and magnetic ion doping (details in Chapter 2.3 and 6.1). The presence of a spin-glass state (SG), ferromagnetism (FM), antiferromagnetism (AFM) and an IMT with doping makes the understanding of physical properties of doped $\mathrm{LaCoO}_{3}$ even more complicated, and especially, in more modern CF theories the covalent mixing of $d$ orbitals and $2 p$ orbitals of oxygen becomes a more and more important issue, which affects both the ground state of the $\mathrm{Co}^{3+}$ ion [43] and its magnetic correlations $[99,2,16]$. For example, the hybridization of $2 p-3 d$ allows the introduction of super-exchange magnetic interactions between the neighboring magnetic moments mediated by oxygen [99], and these magnetic interactions are inherently associated with the electron's conductivity. The relationship between the unusual magnetism observed in doped $\mathrm{LaCoO}_{3}$ and the IMT is still an open question, and a detailed microscopic understanding of the magnetism itself is still lacking.

Dopants invariably cause the lattice to adjust, be it significant on a global scale 
or subtle in a local scale. On the other hand, originating from the special freedom of $\mathrm{Co}^{3+}$, the spin state of $\mathrm{Co}^{3+}$ and its consequences on the magnetism and transport are sensitively affected by small perturbations such as the lattice adjustment. Therefore, the rich physical properties exhibited by the doped $\mathrm{LaCoO}_{3}$ ultimately result from the complicated interplay in between the lattice, magnetism and conductivity. The neutron scattering technique is a perfect tool for investigating these issues because, unlike X-rays, neutrons are scattered strongly by light atoms such as oxygen so that the crystal structure of cobalt oxides can be accurately determined. Neutron scattering also provides direct information on the spatial and temporal spin-spin correlations and the associated magnetic fluctuations and excitations (details in Chap 3). Thus, in the current work, neutron scattering is used to investigate the correlations between the lattice and magnetism in the following three systems,

- Hole doping, $\mathrm{La}_{1-x} \mathrm{~A}_{x} \mathrm{CoO}_{3}\left(\mathrm{~A}=\mathrm{Ca}^{2+}, \mathrm{Sr}^{2+}\right.$ and $\left.\mathrm{Ba}^{2+}\right)$

Holes are introduced by substitution of a trivalent ion, La, with a divalent ion such as $\mathrm{Sr}^{2+}$. Early intensive studies based on the bulk properties showed that the compound, $\mathrm{La}_{1-x} \mathrm{Sr}_{x} \mathrm{CoO}_{3}$, evolves from a spin-glass (SG), insulating state to a ferromagnetic $(\mathrm{FM})$, metallic state at a doping concentration $x \sim 18 \%[34,90]$ (details in Chapter 2.3). In the most recent study, a coexistence of FM and incommensurate (IC) magnetic clusters of both sizes on the $n m$ scale was found to prevail in the SG state via elastic neutron scattering [62]. The IMT is coupled with SG-FM transition and this is understood in the frame of Double exchange (DE) [99]: the FM clusters 
are electrically conductive, so the percolation of FM metallic clusters brings about the IMT and SG-FM transition at the same time. The IC phase, however, is insulating because the AFM spin-spin correlations localize the electrons based on Super exchange [12]. After finding the IC phase, an immediate question is: "What is the origin of the IC phase?" Before answering this challenging question, it is useful to determine whether the magnetic phase separation is unique for $\mathrm{Sr}$ doping. Considering that the elements of $\mathrm{Ca}, \mathrm{Sr}$ and $\mathrm{Ba}$ are the closest neighbors in the same main group (IIA) and are therefore electronically equivalent, in the current work this issue is investigated through a characterization of the magnetic phase separation in all three compounds, $\mathrm{La}_{1-x} \mathrm{~A}_{x} \mathrm{CoO}_{3}\left(\mathrm{~A}=\mathrm{Ca}^{2+}, \mathrm{Sr}^{2+}\right.$ and $\left.\mathrm{Ba}^{2+}\right)$ by measuring the static spin correlations via neutron scattering.

- Isovalent magnetic ion doping, $\mathrm{LaCo}_{1-y} \mathrm{Ni}_{y} \mathrm{O}_{3}$ and $\mathrm{LaCo}_{1-y} \mathrm{Fe}_{y} \mathrm{O}_{3}$

The direct replacement of $\mathrm{Co}^{3+}$ ion with isovalent magnetic ions such as $\mathrm{Ni}^{3+}$ and $\mathrm{Fe}^{3+}$ results in essentially different physical properties. The effects of $\mathrm{Ni}$ doping resemble the hole doping the most: $\mathrm{LaCo}_{1-y} \mathrm{Ni}_{y} \mathrm{O}_{3}$ develops from the SG to FM state; a negative magneto-resistivity coupled with the SG state; and an IMT [60, 29, 3, 41]. However, the IMT occurred at a much higher doping concentration than the SG-FM transition [29]. On the other hand, the $\mathrm{LaCo}_{1-y} \mathrm{Fe}_{y} \mathrm{O}_{3}$ is an insulator regardless how much Fe is doped, and the compounds remain in the paramagnetic state until a weak AFM order sets in at a high doping level [38, 87].

The elements, $\mathrm{Ni}$ and $\mathrm{Fe}$, are both adjacent neighbors to $\mathrm{Co}$, and both trivalent 
ions are magnetic, but why does doping not bring about similar effects? The state of the $\mathrm{Co}^{3+}$ ion must play a critical role. Considering the fact that the ionic sizes of $\mathrm{Fe}^{3+}, \mathrm{Co}^{3+}$ and $\mathrm{Ni}^{3+}$ are almost identical so that the steric effects due to the dopant are negligible, under such circumstances how does the lattice respond to the case when the dopant is $\mathrm{Ni}$ and when it is Fe? Would be the response different? If so, would the differences, which are not due to the steric effects, be correlated with the state of $\mathrm{Co}^{3+}$ and the magnetism of the compounds? In the current work, this is examined by neutron scattering.

On the other hand, the fact that the IMT and SG-FM transition are not coupled in $\mathrm{LaCo}_{1-y} \mathrm{Ni}_{y} \mathrm{O}_{3}$ first poses a question about the microscopic nature of the SG state especially considering the observed magnetic inhomogeneities in the SG states of the hole-doped systems. In the current work, this problem is clarified by measuring the static spin correlations. Secondly, this indicates a different role of magnetism in the IMT for Ni doping than for hole doping. The parent compound $\mathrm{LaNiO}_{3}$ is a good metal with Pauli paramagnetism, i.e., a band metal, which indicates a collective behavior of $d$ electrons based on the band theory. As a contrast, $\mathrm{LaCoO}_{3}$ is a Mott insulator, indicating a localized $d$ electrons of which behavior is well understood by the crystal field theory. Then, for the mixed compound, $\mathrm{LaCo}_{y} \mathrm{Ni}_{1-y} \mathrm{O}_{3}$, how do we understand or describe the behavior of the $d$ electrons, which involves the interactions between the collective $d$ electrons of Ni and localized ones of Co. The FM and IMT transitions would ultimately be attributed to the interaction of $d$ electrons of $\mathrm{Co}^{3+}$ 
ions with dopants, i.e., holes or the $d$ electrons of $\mathrm{Ni}^{3+}$ ions, which determines the spins and carrier mobility at the same time. In the current work this problem is addressed by inelastic neutron scattering and bulk resistivity and magnetization measurements.

- $\mathrm{PrCoO}_{3}$

Finally, one may wonder if the spin-state freedom still survives in other rare-earth perovsike cobaltites, such as in $\mathrm{PrCoO}_{3}$, considering that its crystal structure adopted a lowered symmetry due to the smaller rare-earth ionic size [83, 40]. In the current work, a search for the evidence of thermal spin excitation was carried out via neutron diffraction up to $350 \mathrm{~K}$ and inelastic scattering up to $300 \mathrm{~K}$.

\subsection{Layout of the Dissertation}

The rest of dissertation is arranged as follows: In the next Chapter, the background knowledge and the results of some important previous experiments are provided which lay the base for the discussion of the next chapters. In Chapter 3, the relevant neutron scattering theory is outlined, and the application of neutron scattering and instrumentation are introduced. In Chapter 4, the details about the powder and single crystal sample preparations are provided. In Chapter 5, the magnetic phase separation trends are characterized in the hole doped system, $\mathrm{La}_{1-x} \mathrm{~A}_{x} \mathrm{CoO}_{3}$ $\left(\mathrm{A}=\mathrm{Ca}^{2+}, \mathrm{Sr}^{2+}\right.$ and $\left.\mathrm{Ba}^{2+}\right)$, on single crystals via elastic neutron scattering. A hypothesis to explain the observed magnetic inhomogeneity and the trend with the A-site 
dopant is discussed. In Chapter 6, two major topics about the isovalent magnetic ion doped systems are discussed: (1) in $\mathrm{LaCo}_{1-y} \mathrm{~B}_{y} \mathrm{O}_{3}\left(\mathrm{~B}=\mathrm{Ni}^{3+}\right.$ or $\left.\mathrm{Fe}^{3+}, y \leq 0.4\right)$ the ground state of $\mathrm{Co}^{3+}$ ion after doping are investigated by bulk magnetization and neutron diffraction. It is argued that there is a correlation between the magnetic states and the trigonal distortion. The magnetic correlation is identified to be only ferromagnetic in the SG state of $\mathrm{LaCo}_{1-y} \mathrm{Ni}_{y} \mathrm{O}_{3}$ and the reason for absence of AFM correlation can be also understood by the hypothesis proposed in the hole-doped system. (2) The nature of the magnetic exchange in $\mathrm{LaCo}_{1-y} \mathrm{Ni}_{y} \mathrm{O}_{3}$ is investigated after finding a new low-energy excitation in dilute doped compounds $(y \leq 0.01)$ via inelastic neutron scattering. Two possible scenarios are proposed to interpret the origin of the new excitation. In Chapter 7, a search for the thermal spin excitation in $\mathrm{PrCoO}_{3}$ is carried out by neutron diffraction and inelastic neutron scattering up to $350 \mathrm{~K}$. The absence of a lattice distortion and the low energy spectra both suggested that the transition may occur at a temperature at least above $300 \mathrm{~K}$. In Chapter 8, the experiment results are summarized and based on that the final conclusions are generalized. 


\section{Chapter 2}

\section{Background of Perovskite}

\section{Cobaltites}

\subsection{Crystal Structure}

- Perovskite Structure and Ideal Cubic Symmetry

The perovskite structure can be generalized to a chemical formula $\mathrm{ABX}_{3}$, where $\mathrm{A}$ and $\mathrm{B}$ are cations and $\mathrm{X}$ is an anion. Many oxides adopt this structure $\left(\mathrm{ABO}_{3}\right)$ such as the original perovskite mineral calcium titanium oxide $\left(\mathrm{CaTiO}_{3}\right)$ where the A site is $\mathrm{Ca}^{2+}$ and the $\mathrm{B}$ site is $\mathrm{Ti}^{4+}$. In our studies of perovskite cobaltites the $\mathrm{A}$ cations are trivalent rare earth ions such as $\mathrm{La}^{3+}$ or $\mathrm{Pr}^{3+}$. The $\mathrm{B}$ cation is a transition metal ion, $\mathrm{Co}^{3+}\left(3 d^{6}\right)$. The structure can be described as the corner shared oxygen octahedra $\mathrm{CoO}_{6 / 2}$ with $\mathrm{Co}^{3+}$ ions in the centers of the octahedra and the A-site rare 


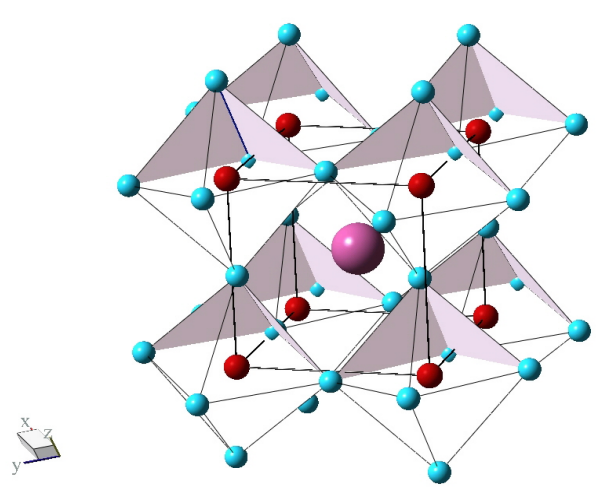

Figure 2.1: The perovskite structure and the $\mathrm{BO}_{6 / 2}$ octahedra

earth ion filling the space in between eight octahedra (Fig.2.1).

The ideal symmetry is cubic with the space group $\operatorname{Pm} \overline{3} \mathrm{~m}$ [68]. Each cubic crystal unit cell corresponds to one chemical unit, i.e. $\mathrm{ABO}_{3}$. For one unit cell (Fig. 2.2): eight $\mathrm{A}$ ions are located at each corner; six oxygen ions are located at the face centers and one B ion is at the body center. The equivalent atomic positions are listed in table 2.1. In this case, it is obvious that $\frac{A-O}{\sqrt{2} B-O}=1$, where A-O and B-O are the equilibrium bond lengths between $\mathrm{A}$ and oxygen ions, and $\mathrm{B}$ and oxygen ions, respectively.

- Tolerance Factor $t$ and Lowered Crystal Symmetries 


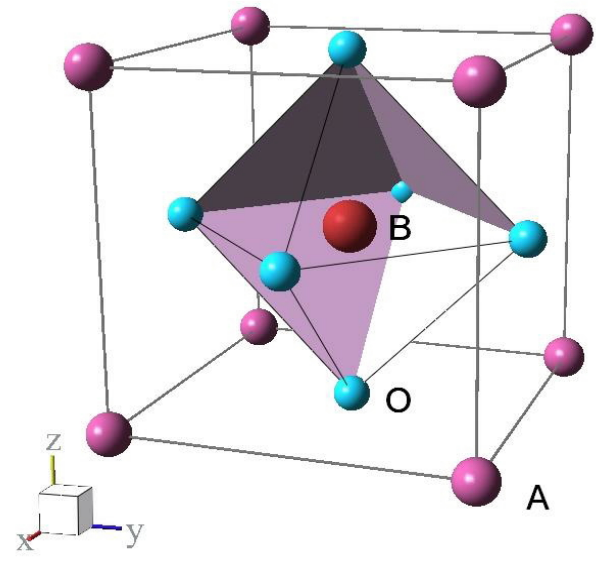

Figure 2.2: The ideal cubic symmetry of the perovskite structure

\begin{tabular}{|l|l|l|}
\hline Site & Multiplicity \& Wyckoff letter & Coordinates \\
\hline A & $1 a$ & $(0,0,0)$ \\
B & $1 b$ & $\left(\frac{1}{2}, \frac{1}{2}, \frac{1}{2}\right)$ \\
O & $3 c$ & $\left(0, \frac{1}{2}, \frac{1}{2}\right)$ \\
\hline
\end{tabular}

Table 2.1: Atomic positions in cubic perovskite $\mathrm{ABO}_{3}$ 


\begin{tabular}{|l|l|l|}
\hline Crystal systems & Space group & Rotation axis \\
\hline tetragonal & $I 4 / m c m$ & {$[001]$} \\
rhombohedral & $\mathrm{R} \overline{3} \mathrm{c}$ & {$[111]$} \\
orthorhombic & Pnma & {$[110]$} \\
\hline orthorhombic & Imma & {$[101]$} \\
\hline
\end{tabular}

Table 2.2: The lowered symmetries and the corresponding $\mathrm{BO}_{6 / 2}$ rotations

In reality, however, due to the mismatch of the ionic sizes of $\mathrm{A}$ and $\mathrm{B}$ ions, the ideal cubic symmetry is often not realized. Instead, the lattice adjusts to the difference by adopting a lower crystal symmetry, and the perfect cube undergoes a deformation and becomes a pseudo-cube. Consequently, $\frac{A-O}{\sqrt{2} B-O} \neq 1$, this ratio is actually defined as the tolerance factor, $t$, and it is used to quantify the mismatch [28]. To calculate the value of $t$, the empirical ionic radii are used which are obtained from the X-ray data at room temperature under atmospheric pressure [77]. As $t>1$, the accommodated structure is hexagonal stacking, where $\mathrm{AO}_{3}$ close-packed planes form hexagonal polytypes [27]. As $t<1$, the possible symmetries are tetragonal, rhombohedral and orthorhombic. Each symmetry can be obtained by a cooperative rotation of $\mathrm{BO}_{6 / 2}$ octahedra around one of the axes of a cubic cell [27], and Table 2.2 lists the symmetries and the corresponding rotation axes.

For the perovskite cobaltites, to the best of our knowledge, whether the value of $t$ is obtained by using the empirical ionic radii or by using the average bond lengths of $\mathrm{A}-\mathrm{O}$ and $\mathrm{B}-\mathrm{O}$ obtained from Rietveld refinement, $t$ is found to be smaller than 1. In this case, for $\mathrm{LaCoO}_{3}$ and its doped compounds, the most common crystal 


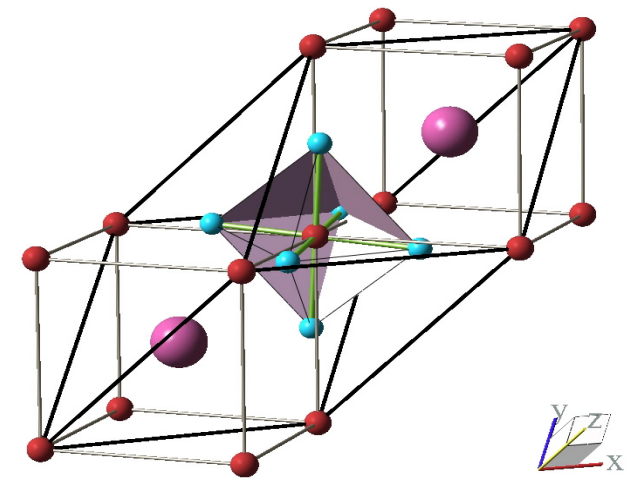

Figure 2.3: Rhombohedral unit cell and its two constituent psudocubic cells.

symmetry is rhombohedral $(\mathrm{R} \overline{3} \mathrm{c})$, while for the other rare earth perovskite cobaltites such as $\mathrm{PrCoO}_{3}$ and the hole doped compound with smaller ion of $\mathrm{Ca}, \mathrm{La}_{1-x} \mathrm{Ca}_{x} \mathrm{CoO}_{3}$ $[63,44,13]$, the smaller A-site ionic size results in a lower symmetry, orthorhombic (Pnma). For R $\overline{3} \mathrm{c}$, one rhombohedral unit cell is comprised of two chemical units or two pseudo-cubic cells, and for Pnma one crystal unit cell contains four chemical units or four pseudo-cubic cells. Figs. 2.3 and 2.4 show the crystal unit cell (black outline) and the constituent pseudo-cubic cells (grey outline) for R $\overline{3} \mathrm{c}$ and Pnma, respectively, and the following Tables 2.3 and 2.4 list the corresponding equivalent atomic positions. 


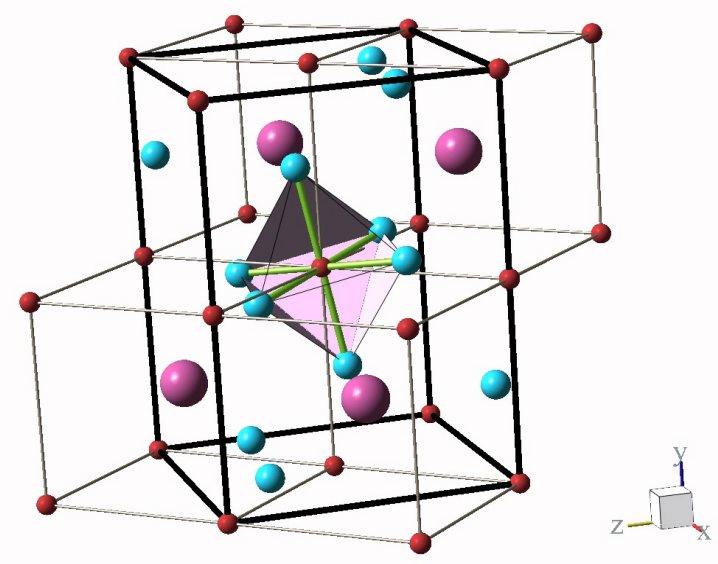

Figure 2.4: Orthorhombic unit cell and its four constituent psudocubic cells.

\begin{tabular}{|l|l|l|}
\hline Site & Multiplicity \& Wyckoff letter & Coordinates \\
\hline A & $2 a$ & $\left(\frac{1}{4}, \frac{1}{4}, \frac{1}{4}\right)$ \\
B & $2 b$ & $(0,0,0)$ \\
O & $6 e$ & $\left(-x, \frac{1}{2}+x, \frac{3}{4}\right)$ \\
\hline
\end{tabular}

Table 2.3: Atomic positions in rhombohedral perovskite $\mathrm{ABO}_{3}$ based on rhombohedral setting.

\begin{tabular}{|l|l|l|}
\hline Site & Multiplicity \& Wyckoff letter & Coordinates \\
\hline $\mathrm{A}$ & $4 c$ & $\left(x_{A}, \frac{1}{4}, z_{A}\right)$ \\
$\mathrm{B}$ & $4 a$ & $(0,0,0)$ \\
$\mathrm{O}(1)$ & $4 c$ & $\left(x_{o 1}, \frac{1}{4}, z_{o 1}\right)$ \\
$\mathrm{O}(2)$ & $8 d$ & $\left(x_{o 2}, y_{o 2}, z_{o 2}\right)$ \\
\hline
\end{tabular}

Table 2.4: Atomic positions in orthorhombic perovskite $\mathrm{ABO}_{3}$. 


\subsection{Thermal Driven Spin-State Transition}

From our previous discussion of the crystal structure, we know that the transition metal ion, in this case $\mathrm{Co}^{3+}\left(3 d^{6}\right)$, is located in the center of an octahedron formed by six oxygen anions. Under the simple cubic symmetry (although the actual symmetry is trigonal or rhombohedral), the regular octahedral crystal field (described by the point symmetry symbol $\left.O_{h}\right)$ splits the five degenerate $d$ orbitals $\left(d_{x y}, d_{x z}, d_{y z}, d_{x^{2}-y^{2}}\right.$

and $\left.d_{z^{2}}\right)$ into two classes: three $t_{2 g}$ orbitals at the lower energy $\left(d_{x y}, d_{x z}, d_{y z}\right)$ for which the $d$ orbitals point between the $x, y$ and $z$ axes and two $e_{g}$ orbitals at higher energy $\left(d_{x^{2}-y^{2}}\right.$ and $\left.d_{z^{2}}\right)$ for which the $d$ orbitals point along these axes [12]. Fig. 2.5 demonstrates the five $d$ orbitals of the transition metal ion and its surrounding oxygen environment. Notice the orientation of the orbitals determines the overlapping of the electron clouds of oxygen's $2 p$ and transition metal's $d$ so that the energy levels of $d$ orbitals are split: the more overlapping the higher energy. If there are no more than three $d$ electrons, the electron will fill up the low $t_{2 g}$ orbitals, but if there are more than three $d$ electrons, then the way to occupy the $t_{2 g}$ and $e_{g}$ orbitals depends on the competition between the crystal field energy, which is the energy difference between $t_{2 g}$ and $e_{g}$ orbitals, and the Coulomb pairing energy (sometimes it is also referred to as the Hund's energy, orbital pairing energy or the interatomic exchange, they all mean the energy cost to put two electrons with opposite spin into a single orbital). For example, if the crystal field energy is lower than the pairing energy, the electrons will fill up the orbitals singly first and then doubly. On the other hand, if the crystal 


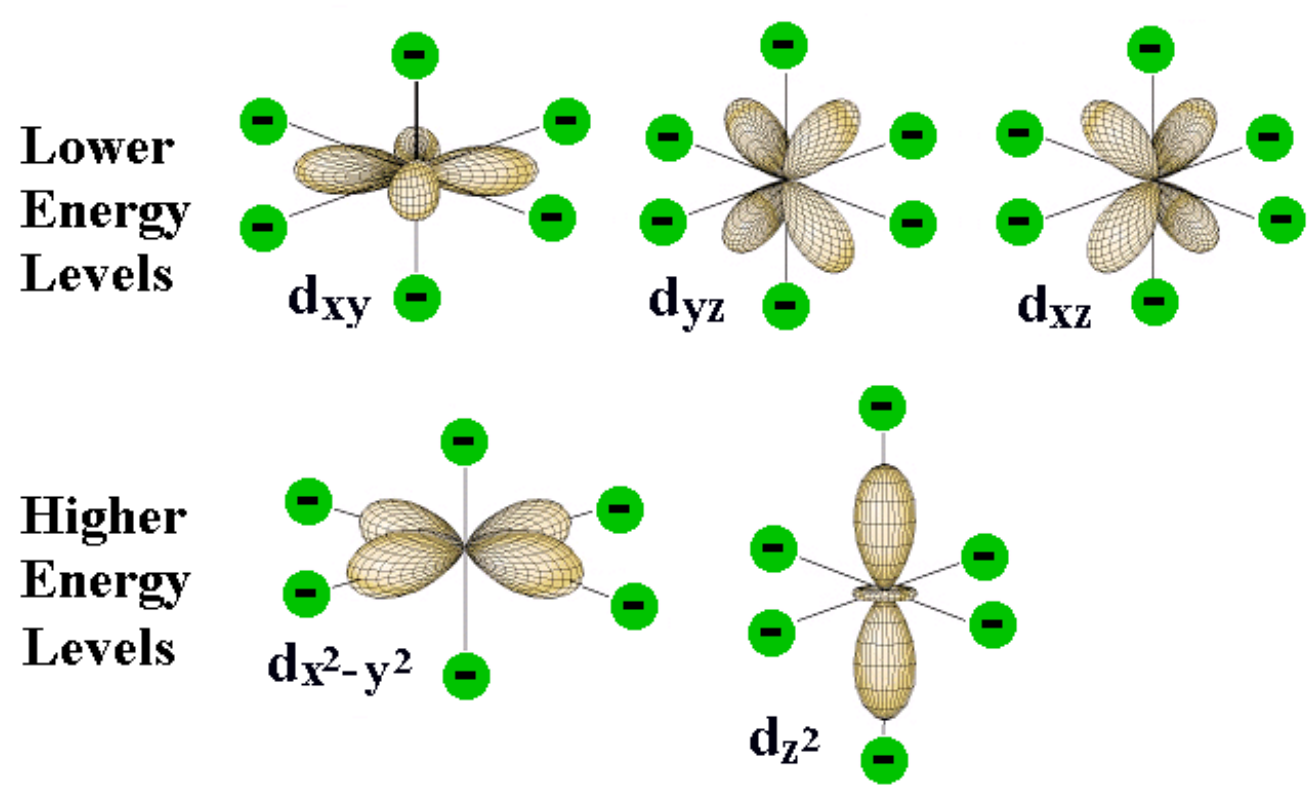

Figure 2.5: $d$ orbitals and the octahedron crystal field levels [18].

field is higher than the pairing energy, the electrons will first fill up the lowest three $t_{2 g}$ orbitals and then start to occupy the $e_{g}$ orbitals [12].

What makes $\mathrm{LaCoO}_{3}$ interesting is that these two energy scales are comparable in the specific crystal field accommodated by this compound. Therefore, how the six electrons of $\mathrm{Co}^{3+}$ ion occupy the split orbitals does not make a significant difference in energy. Fig. 2.6 is a schematic of different ways that six electrons occupy the orbitals. The ground state has an electronic configuration of $t_{2 g}^{6} e_{g}^{0}$ where all the electrons are paired so the net spin value is equal to zero. This state is referred to the low-spin (LS) state. There are two excited states: one with configuration, $t_{2 g}^{5} e_{g}^{1}$ so $\mathrm{S}=1$ and it is called an intermediate spin state (IS), and the other with $t_{2 g}^{4} e_{g}^{2}$ so $\mathrm{S}=2$ called a high 


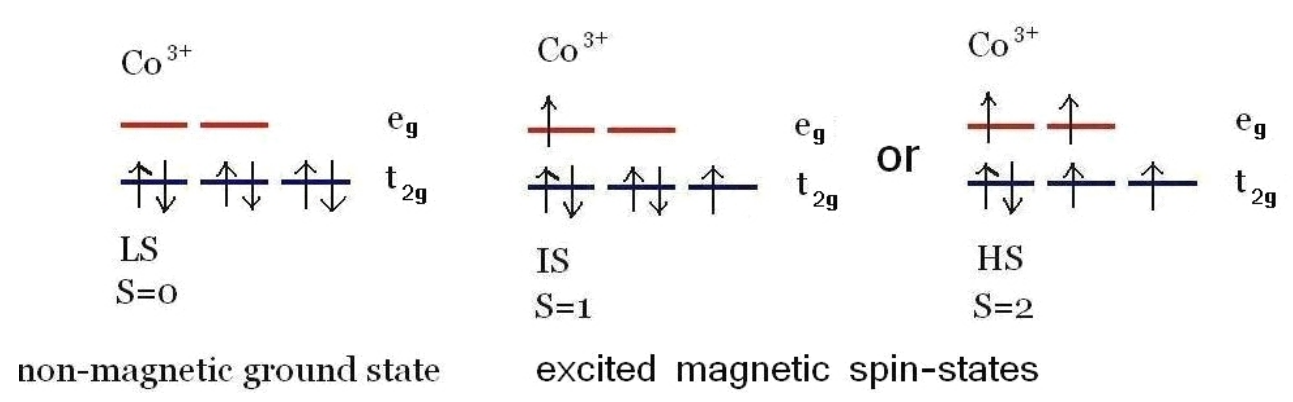

Figure 2.6: Electronic configurations of $\mathrm{Co}^{3+}$ ion at the LS, IS and HS state.

spin state (HS). The estimated spin-gap energy by the optical spectroscopy and the theoretical calculation is as small as $\sim 30 \mathrm{meV}$ [91]. This means that upon warming up to $\sim 100 \mathrm{~K}$, a transition from the spin-zero state (LS) to the magnetic states (IS or/and HS) occurs. This transition "switches" $\mathrm{LaCoO}_{3}$ from a non-magnetic Mott insulator to a paramagnetic semiconductor. As the temperature rises to $\sim 500 \mathrm{~K}$, a second transition from a localized to an itinerant state occurs, which leads to a insulator-metal transition (IMT). Fig. 2.7 shows the temperature dependence of the electrical resistivity (upper panel) and the magnetic susceptibility (lower panel) for $\mathrm{LaCoO}_{3}[91]$. For the resistivity data, two broad drops appeared around $100 \sim 200 \mathrm{~K}$ and $500 \sim 600 \mathrm{~K}$, corresponding to the two transitions mentioned above, respectively. On the other hand, these two transitions were also identified in the bulk magnetic susceptibility by two broad peaks centered at $\sim 100 \mathrm{~K}$ and $\sim 600 \mathrm{~K}$. For the rise from $\sim 30$ to $100 \mathrm{~K}$, it could be caused by an AFM ordering, but no AFM Bragg peaks was observed in the neutron diffraction measurements $[42,85,69]$ and this rise appears the same in both zero-field-cooled and field-cooled data. Thus, it is caused 


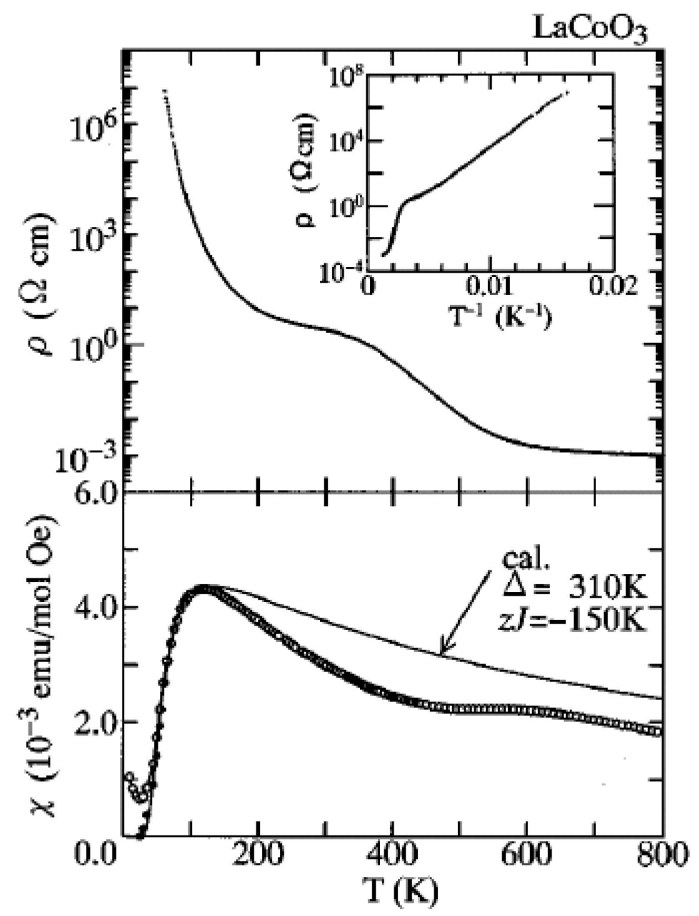

Figure 2.7: Bulk electrical resistivity (upper panel) and magnetic susceptbility (lower panel) for a crystal of $\mathrm{LaCoO}_{3}$ as a function of temperature. Data were obtained from ref [91]. 

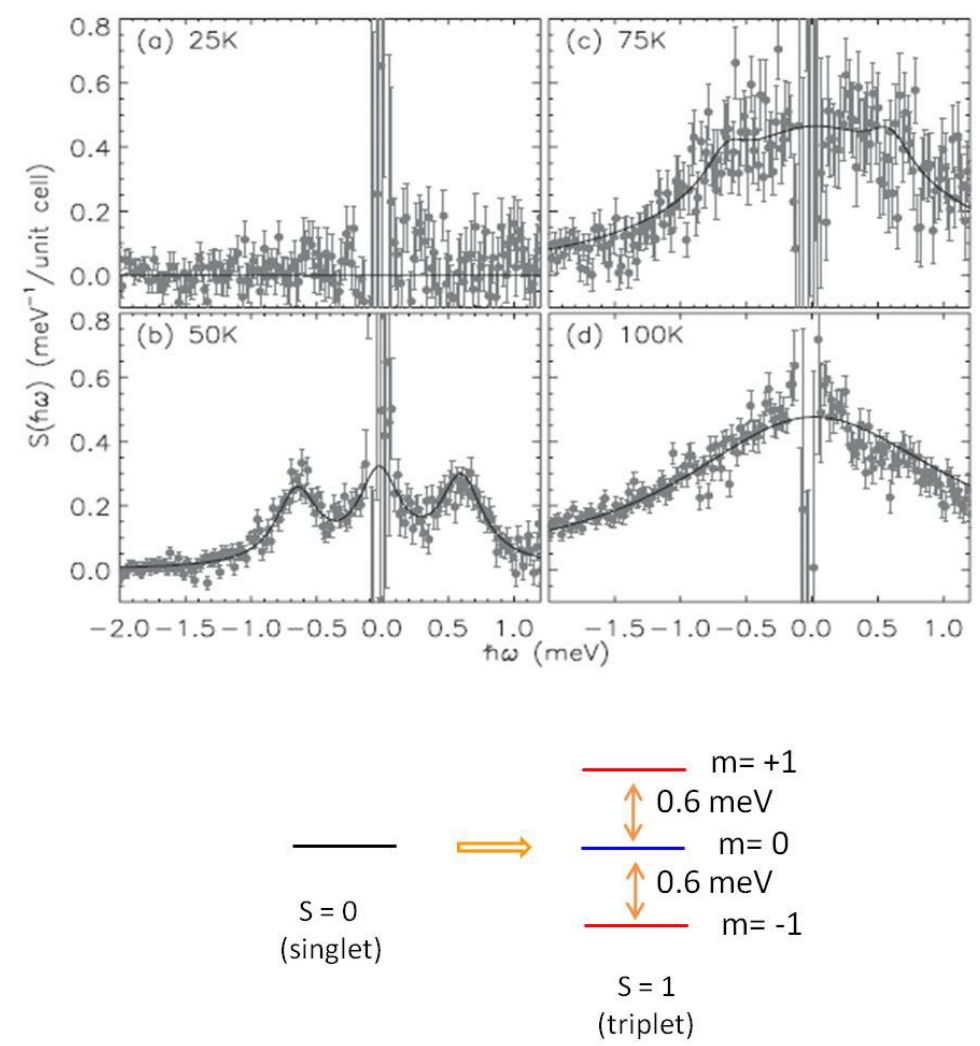

Figure 2.8: The low energy spectra of the powder sample $\mathrm{LaCoO}_{3}$ at different temperatures, after ref [61]. The following schematic shows the energy transfer of 0.6 meV correspondes to the spin-flip transition within the $\mathrm{S}=1$ manifold.

by the increased population of the magnetic $\mathrm{Co}^{3+}$ ions as the result of the spin-state transitions. The Curie-Weiss curve below $\sim 30 \mathrm{~K}$ is argued to be caused by the surface magnetic cobalt ions $[93,76]$. The closed circles below $100 \mathrm{~K}$ are the result of the subtraction from these Curie-like impurities.

The spin state transition was observed in a number of other experiment methods, such as infrared spectroscopy, Raman spectroscopy and electron spin resonance measurements $[92,24,35,58]$. By using neutron scattering technique, a low energy 
excitation associated with this transition was observed in our previous studies [61]. Fig. 2.8 is the low energy spectrum for the powder sample of $\mathrm{LaCoO}_{3}$. Upon warming to $50 \mathrm{~K}$, an excitation at $\sim 0.6 \mathrm{meV}$ is clearly shown. As the temperature continues rising to $\sim 70 \mathrm{~K}$, a center quasi-elastic intensity rises up significantly. By $100 \mathrm{~K}$, the spectrum is dominated by the quasi-elastic intensity. It is found that the $0.6 \mathrm{meV}$ excitation corresponds to a spin-flip transition within the $S=1$ energy manifold [66] and this is shown as a schematic following Fig. 2.8. Notice that both the $0.6 \mathrm{meV}$ excitation and the quasi-elastic peaks appear quite broad in energy transfer space.

Further investigations confirmed that both the $0.6 \mathrm{meV}$ excitation and the quasielastic intensities were magnetic in nature. In reciprocal space the intensity of the 0.6 meV excitation only follows the magnetic form factor of $\mathrm{Co}^{3+}$ ions, and there is no other $\vec{Q}$ dependence. However, the center quasi-elastic intensity shows up as a broad peak and it only occurs at the Bragg positions and the positions half-way between Bragg peaks [61]. Fig. 2.9 is the inelastic neutron scattering data on the single crystal $\mathrm{LaCoO}_{3}$ at $55 \mathrm{~K}$ (blue) and $100 \mathrm{~K}$ (red). Panel (a) and (b) are the energy scans at 55 and $100 \mathrm{~K}$ subtracted from the data at $4 \mathrm{~K}$ at the constant $\mathrm{Q}$ positions $(0.5,0.5,0)$ and $(0,0,1.1)$. The $0.6 \mathrm{meV}$ excitation is visible in both scans. Panel (c) and (d) are the $\mathrm{Q}$ scans at the constant energy transfer of $0.6 \mathrm{meV}$ along the diagonal direction through $(0.5,0.5,0.5)$ and the transverse direction through $(0,0,1.1)$.

The magnetic peaks at the Bragg positions indicate a ferromagnetically coupled spin-spin correlation (FM) and on the other hand the peaks at the half-way positions 


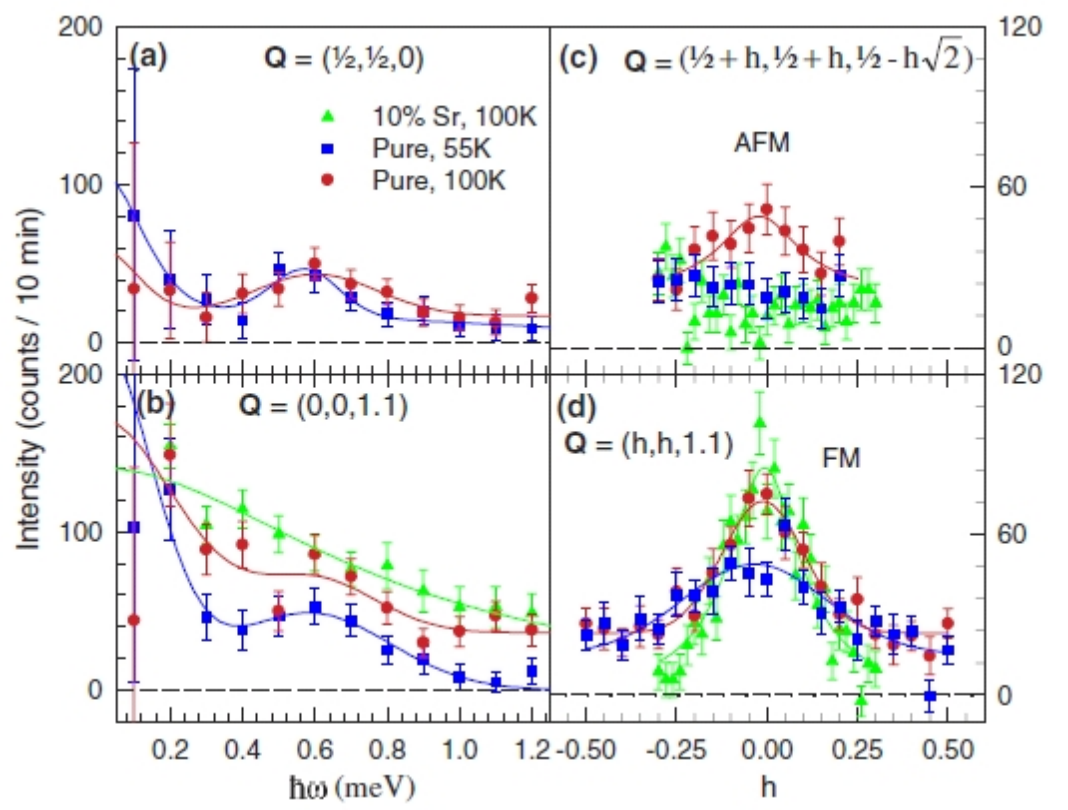

Figure 2.9: Inelastic neutron scattering data obtained from the single crystals of $\mathrm{LaCoO}_{3}$ and $\mathrm{La}_{0.9} \mathrm{Sr}_{0.1} \mathrm{CoO}_{3}$ at SPINS at 55 and $100 \mathrm{~K}$. After ref [61].

indicate an AFM correlation [61]. Based on the properties of Fourier transform of a Gaussian function, the broader the peak in energy transfer space - the shorter the life-time; similarly, the broader the peak in reciprocal space - the shorter the correlation length in real space. Thus, the above two experiments not only indicated the occurrence of the thermal spin excitations but also the existence of FM and AFM correlations which were short-range ordered and short-time lived. 


\subsection{Hole Doping Effects and Incommensurate Mag-}

\section{netic Phase in $\mathrm{La}_{1-x} \mathrm{Sr}_{x} \mathrm{CoO}_{3}$}

A hole is introduced by replacing $\mathrm{La}^{3+}$ with a divalent ion such as $\mathrm{Sr}^{2+}$. The introduction of holes changes the magnetic and transport properties significantly. As an example, Fig. 2.10 shows the magnetic phase diagram of $\mathrm{La}_{1-x} \mathrm{Sr}_{x} \mathrm{CoO}_{3}$ [49]. As it can be seen when doping concentration increases, the system quickly enters a spin-glass state $(x \sim 5 \%)$ and then a FM state $(x \sim 18 \%)$.

The spin-glass state, however, is not a simple system. Our previous elastic neutron scattering on the single crystal samples of $\mathrm{La}_{1-x} \mathrm{Sr}_{x} \mathrm{CoO}_{3}$ identified a coexistence of the incommensurate (IC) and FM phases on a nanometer length scale [62]. Fig. 2.11 (a) is an elastic neutron scattering contour map for $\mathrm{La}_{0.85} \mathrm{Sr}_{0.15} \mathrm{O}_{3}$ in the $(h h l)$ plane in momentum space at T $\sim 8 \mathrm{~K}$. The strongest intensity at the center is at the (001) Bragg peak position. It appears much broader below the magnetic transition temperature. Therefore, the broadened intensity is magnetic and indicates a short-range static FM order. More interestingly, four broad satellite peaks appear around the Bragg peak along (111) and (11-1) directions. These satellite peaks are confirmed to be magnetic and their locations are not commensurate with the crystal lattice periodicity, and thus they are referred as incommensurate peaks (IC). The order parameter of IC can be independent with the one of FM peak, and as doping level $x \geq 0.15$, the onset temperature of IC starts to be a lower temperature than the one of the FM 


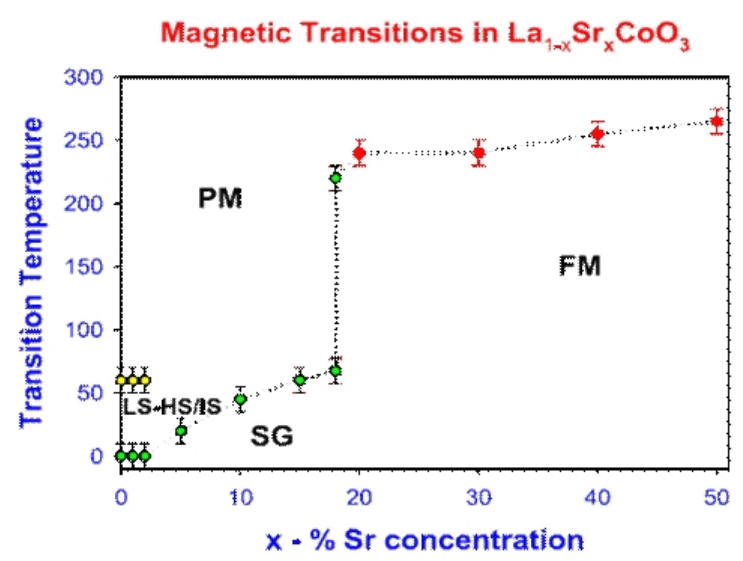

Figure 2.10: Magnetic phase diagram of $\mathrm{La}_{1-x} \mathrm{Sr}_{x} \mathrm{CoO}_{3}$ [49].

signal. Therefore, it is argued that these satellite peaks indicate the existence of an incommensurate magnetic phase [62].

Therefore, the short-range (at the order of the distance between the nearest Co ions) and short-time lived AFM and FM correlations in the pure compound, $\mathrm{LaCoO}_{3}$, appear to settle down to static short-range (at the order of $n m$ ) IC and FM phases in $\mathrm{La}_{1-x} \mathrm{Sr}_{x} \mathrm{CoO}_{3}$.

\subsection{Hole-Doping Induced Magnetic $\mathrm{Co}^{3+}$ Ion in the IS State}

To balance the valence, $\mathrm{Sr}^{2+}$ doping inevitably transfers the same quantity of $\mathrm{Co}^{3+}$ as doping to $\mathrm{Co}^{4+}$,i.e., $\mathrm{La}_{1-x}^{3+} \mathrm{Sr}_{x}^{2+} \mathrm{Co}_{x}^{4+} \mathrm{Co}_{1-x}^{3+} \mathrm{O}_{3}^{2-}$. Due to its odd number of electrons $\left(3 d^{5}\right)$, the $\mathrm{Co}^{4+}$ ion is magnetic regardless of its spin state $\left(\mathrm{LS}: \mathrm{S}_{L S}=\frac{1}{2}\right.$ with $t_{2 g}^{5} e_{g}^{0}$; 


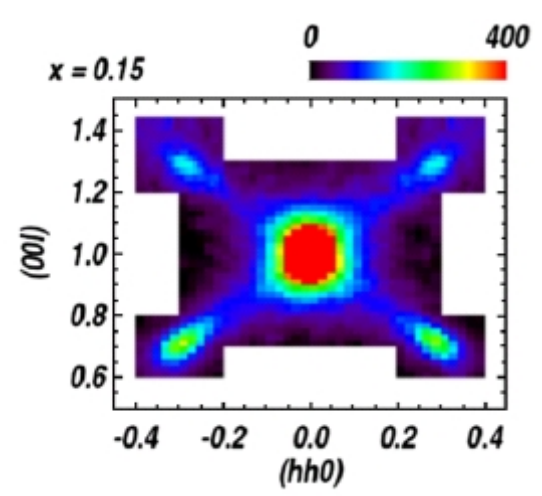

Figure 2.11: The elastic neutron scattering contour map in the (hhl) plane for the single crystal of $\mathrm{La}_{0.85} \mathrm{Sr}_{0.15} \mathrm{CoO}_{3}$. The figure is from ref [62].

IS: $\mathrm{S}_{I S}=\frac{3}{2}$ with $t_{2 g}^{4} e_{g}^{1} ; \mathrm{HS}: \mathrm{S}_{H S}=\frac{5}{2}$ with $\left.t_{2 g}^{3} e_{g}^{2} ;\right)$. Thus, following a naive reasoning, the complex magnetic states mentioned in the last section might be just caused by $\mathrm{Co}^{4+}$ ions.

To find out whether this is true or not, an investigation on the spin states of trivalent Co ion while the doping level is still very low may be useful. First, it was noticed from the bulk magnetic susceptibility $\chi(\mathrm{T})$ measurements, that lightly Srdoping quickly suppressed the thermal spin state transition [91]. Fig. 2.12 is the $\chi$ as a function of temperature for $\mathrm{La}_{1-x} \mathrm{Sr}_{x} \mathrm{CoO}_{3}, x=0,0.002,0.005$ and 0.01 . It is obvious that the rise of susceptibility from $\sim 30$ to $100 \mathrm{~K}$ due to thermal spin excitations is less pronounced, while on the other hand, the low-temperature Curie-Weiss-like behavior is strengthened. As the doping level increases, this trend becomes even more obvious. The saturated magnetic moment at this low temperature, where the Curie-Weiss behavior dominates, however, is found out to be unexpected large. The saturated spin value can be estimated by measuring the magnetic field dependence of 


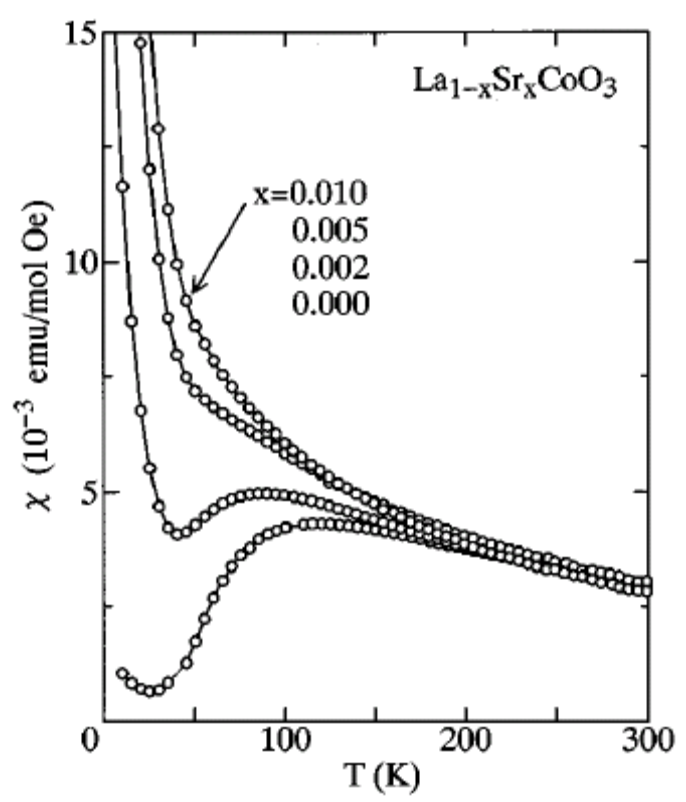

Figure 2.12: The temperature dependence of the magnetic susceptibility of lightly $\mathrm{Sr}$ doped compounds $\mathrm{La}_{1-x} \mathrm{Sr}_{x} \mathrm{CoO}_{3}(x=0,0.002,0.005,0.010)$. After ref. [91].

the magnetization. In $\mathrm{La}_{0.998} \mathrm{Sr}_{0.002} \mathrm{CoO}_{3}$ it was found to be $S \sim 10 \mu_{B}$ / hole where the value of $g$ is taken as 2.18 [91]. Such a large magnetic moment cannot be accounted for by the contribution only from $\mathrm{Co}^{4+}$ ions, and it was interpreted as the total spin value of a magnetic cluster which is formed by a $\mathrm{Co}^{4+}$ and its nearest $\mathrm{Co}^{3+}$ neighbors. $\mathrm{Co}^{3+}$ ions, therefore, ar already on the magnetic states (IS or HS) even without warming. An investigation of the local atomic properties provided a more straightforward evidence of the existence of magnetic $\mathrm{Co}^{3+}$ and furthermore indicated that it is in the IS state $[49,50]$. This was carried out by Pair Density Function (PDF) analysis where the neutron diffraction data were Fourier transformed into real space (The details about PDF and the neutron scattering will be discussed in Chap 3). 
Fig. 2.13 is the static PDF of $\mathrm{La}_{1-x} \mathrm{Sr}_{x} \mathrm{CoO}_{3}$ at $300 \mathrm{~K}$ in four compositions [49], which are at doping level $x=10 \%$ up to $50 \%$ and spread out from spin-glass to FM state according to the phase diagram (Fig. 2.10). The peak position corresponds to the distance between one pair of atoms in real space, i.e., a bond length, and the area under a peak is proportional to the atomic coordination. The closest bond lengths in the perovskite structure are the Co-O bonds in one octahedron (Fig. 2.3). This bond length is typically around $1.9 \AA$ and it corresponds to the first peak in the shown PDF. The rhombohedral (sometimes it is also referred as trigonal) crystal

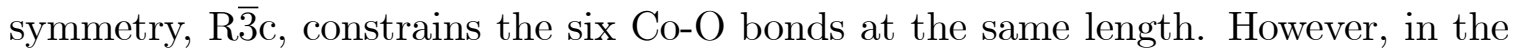
local structure the first PDF peak clearly splits into two peaks with one at a higher distance of $\sim 2.1 \AA$. This indicates that the Co-O bonds in one octahedron are split into long and short bonds. A Jahn-Teller (JT) distortion can cause such a local lattice distortion, and among the three spin states of $\mathrm{Co}^{3+}$ ions, the IS state is subject to JT distortion, where the octahedron is prolonged along one of the orthogonal axis (shown in Fig. 2.14) and results in four short Co-O bonds in the plane and two long bonds perpendicular. For the three spin states of $\mathrm{Co}^{3+}$ ion, only the IS state is JT active. Therefore, the observed splitting indicated the existence of static $\mathrm{IS} \mathrm{Co}^{3+}$ ions.

The Jahn-Teller mechanism described a situation where the magnetic properties can influence the lattice symmetry [36]. If the electronic state of a molecule or complex is orbitally degenerate, then there is always at least one mode of distortion that the molecule or complex can apply to break the degeneracy so as to lower the electronic 


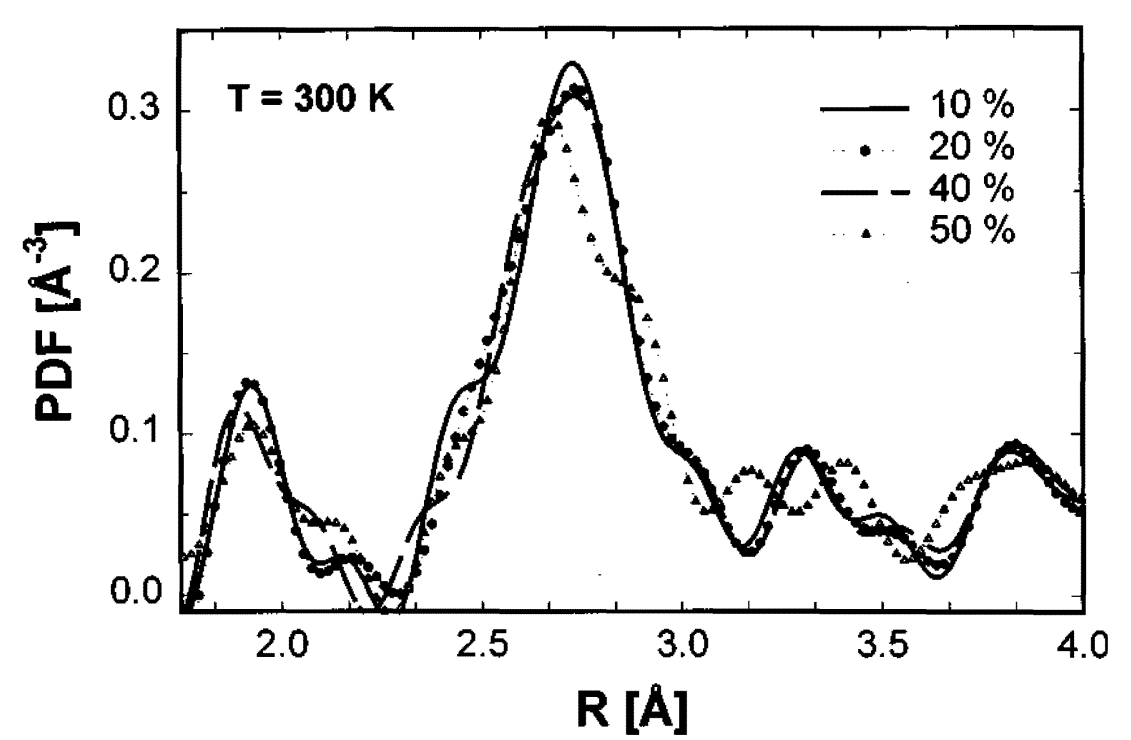

Figure 2.13: The pair density functions (PDF) of $\mathrm{La}_{1-x} \mathrm{Sr}_{x} \mathrm{CoO}_{3}$ for $x=0.1,0.2,0.4$ and 0.5 at $300 \mathrm{~K}$. After ref. [49].

energy. As an example, Fig. 2.14 shows the JT effect in $\mathrm{LaMnO}_{3}\left(\mathrm{Mn}^{3+}: 3 d^{4}\right)[12]$. For the complex of octahedron $\mathrm{MnO}_{6 / 2}$, the ground state of $\mathrm{Mn}^{3+}$ ion in a cubic crystal field is $t_{2 g}^{3} e_{g}^{1}$. After the octahedron is prolonged in the way shown in the figure, two of the Co-O bonds become relatively longer than the other four. This directly changes the degree of $2 p-3 d$ orbital overlapping, and as a result, the $e_{g}$ and $t_{2 g}$ orbitals' degeneracy is broken. The distortion lowers the electronic energy because the net energy change is negative: the singly occupied $e_{g}$ level is lowered and two of the $t_{2 g}$ orbitals are lowered with only one of them raised. Now, for the IS state of $\mathrm{Co}^{3+}$ ions, $\left(t_{2 g}^{5} e_{g}^{1}\right)$, the $e_{g}$ orbitals are also singly occupied, and there is one hole in $t_{2 g}$ orbitals. Such a configuration is possible for the net electronic energy change to be negative after the distortion. 

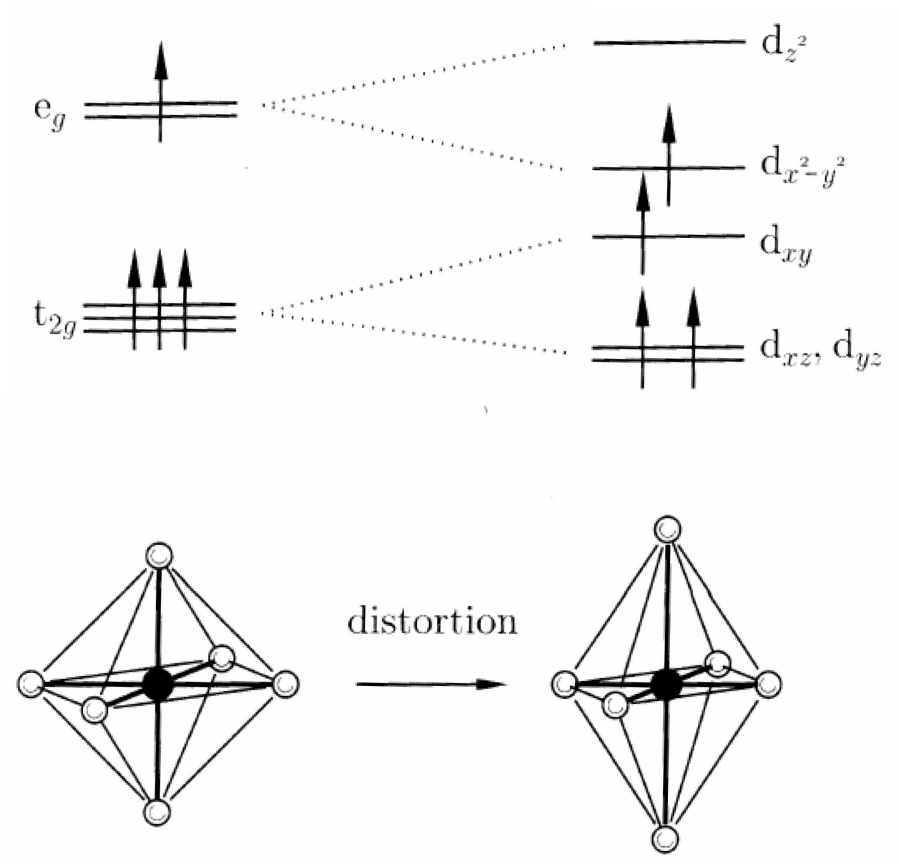

Figure 2.14: The Jahn-Teller distortion for $\mathrm{Mn}^{3+}\left(3 d^{4}\right)$. The distortion prolongs the octahedron and breaks the degeneracy of the $t_{2 g}$ and $e_{g}$ orbitals. The figure is from ref [12]. 


\section{Chapter 3}

\section{Neutron Scattering}

\subsection{Introduction}

Our ability to 'see' an object is determined by two factors: one is that we have to have some kind of instrument or apparatus; the other is that there has to be some "medium" that this instrument can use. For example, in the daily life, we see the world around us by using our bare eyes, which is our instrument, via visible light, which is the medium. The wavelength of the medium sets up an intrinsic limitation on the scale of the objects that can be seen. That is, the wavelength of the medium has to be smaller than or at least about the same as the length scale of the object. Therefore, by using the visible light $\left(\lambda_{\text {violet }} \sim 4 \times 10^{3} \AA-\lambda_{\text {red }} \sim 7 \times 10^{3} \AA\right)$ our human

eyes can see an object at a minimum length scale at about $10^{4} \AA$. For anything smaller than that we just simply cannot see it with our bare eyes. 
For the purpose of study of the matter in an atomic scale, the typical length, such as the atom spacings, is at the order of about angstrom $(\AA)$. For this length scale, the wavelength of the visible light is obviously too large and hence some other medium have to be used. So far there are three medium that are commonly used: X-rays, electron beams and neutron beams. Among these three, neutron beams have several significant advantages.

First the neutron scattering technique is an ideal tool for studying the atomic properties and the dynamics at the same time. Neutron beams are particle waves, so they follow the de Broglie relation, $E=\frac{h^{2}}{2 m \lambda^{2}}$, where $h$ is the Planck constant and $m$ is the mass of neutron. This gives rise to $E=\frac{81.81}{\lambda^{2}}$, where the unit for the energy of neutrons is milli-electron-volts $(\mathrm{meV})$ and the unit for the wavelength is angstrom $(\AA)$. Angstrom is a typical length scale for atomic distances and the energy at meV is comparable with the kinetic energy of atoms in a solid. This means that as neutrons probe the atomic structures, at the same time they measure the dynamics of the atoms. In other words, neutron scattering provides the information about where the atoms are and at the same time what the atoms do. As a comparison, X-rays are electromagnetic waves and follow the Planck's relation: $E=\frac{h c}{\lambda}\left(i . e ., E(e V)=\frac{1.24 \times 10^{4}}{\lambda(\dot{A})}\right)$, this results when the wavelength of the X-rays is about angstrom, the corresponding energy is already as high as the order of killo-electron-volts (keV) or mega-electronvolts $(\mathrm{MeV})$, which is way too high for study the dynamics and indeed "kill" the samples. 
Secondly, because neutrons interact with the nuclei of the sample via a nuclear interaction and not with the electrons of the sample, this makes neutron scattering especially useful in the following three aspects,

(a) Neutrons are highly penetrating, so the bulk properties are measured. The nuclear interaction is a very short range force $\left(\sim \mathrm{fm}, 1 \mathrm{fm}=10^{-15} \mathrm{~m}\right)$, so neutrons can travel all the way into the matter and probe the bulk properties. As a comparison, electron beams and X-rays are mainly scattered by the electron clouds so the penetrating depths of both are very short, and as a result only the surface properties are probed.

(b) Light elements such as Hydrogen and Oxygen are "visible" for neutrons. X-rays are mainly scattered by the electrons so the scattering cross-sections systematically increase with the number of the electrons of the atom, in other words, the atomic number. Thus the light elements are hard to be detected if the sample also contains heavy atoms, because the feeble scattering from the light elements will be swamped by those from heavy atoms. For neutrons, however, because the scattering is via nuclear interactions, the neutron scattering cross sections are not dependent on the number of the electrons of the element. In fact, the cross sections have little correlation with the atomic number, and the scattering by the light atoms such as Hydrogen and Oxygen is not weak at all compared with the ones by heavy atoms. Thus, neutron scattering has been used to precisely locate hydrogen atom and determine the molecular structure. Also neutrons were used to determine the oxygen positions accu- 
rately for the first time after one of the high-temperature superconductors discovered contained copper and yttrium.

(c) Neutrons' cross sections are isotope sensitive. Because of the nature of the nuclear interactions, the interaction strengths vary if the nuclei are different. For example, the nuclei of ${ }^{1} \mathrm{H}$ which contains only one proton has a huge scattering cross section (82.03 barn). However, for the nuclei of isotope $\mathrm{D}\left({ }^{2} \mathrm{H}\right)$ which contains one proton and one neutron, the scattering cross section is only 7.63 barn.

Finally, neutrons have a magnetic moment, so they also interact with the unpaired electrons of the matter via a magnetic dipole interaction. This provides the direct information on the spin-spin correlations. For example, the first direct evidence of anti-ferromagnetic ordering was provided only after neutron scattering techniques were applied.

\subsection{Neutron Scattering Theory Highlights}

The theory of neutron scattering has been derived in detail in several books [80, $51,78]$. In this section two important results about the pair density function and the Bragg diffraction are introduced. The discussions are not aimed for detailed derivations of the formulae but attempt to present a guideline about how the two results are developed from the general nuclear scattering differential cross-section.

1. General nuclear scattering law 
In neutron scattering experiments, what is measured is the intensity of neutrons scattered by the sample as a function of neutron momentum transfer $\vec{Q}\left(\vec{Q}=\vec{k}-\overrightarrow{k^{\prime}}\right)$ and energy transfer $\hbar \omega\left(\hbar \omega=E-E^{\prime}\right)$ normalized by the flux of incident neutrons. The scattered intensity can be expressed in terms of a quantity known as the partial differential cross-section, $\frac{d^{2} \sigma}{d \Omega d E}$, which evaluates the intensity of scattered neutron per incident neutron in a small solid angle $d \Omega$ in a given direction with the final energy between $E^{\prime}$ and $E^{\prime}+d E$.

The general expression for $\frac{d^{2} \sigma}{d \Omega d E}$ by any assembly of nuclei has been mathematically solved, and it is

$$
\frac{d^{2} \sigma}{d \Omega d E}=\frac{1}{2 \pi \hbar} \frac{k^{\prime}}{k} \sum_{j, k} b_{j} b_{k} \int_{-\infty}^{\infty}\left\langle e^{-i \vec{Q} \cdot \overrightarrow{r_{k}}(0)} e^{i \vec{Q} \cdot \overrightarrow{r_{j}}(t)}\right\rangle e^{-i \omega t} d t
$$

Where the sum occurs over all pairs of atoms $j$ and $k, N$ is the total number of nuclei in the sample and $b_{j}$ is the scattering length of the nucleus labeled $j$. The scattering length, $b_{j}$, measures the strength of the interaction between the neutron and the isolated nucleus, and it is related with the scattering cross section, $\sigma$, as $\sigma=4 \pi b^{2}$.

2. Coherent and incoherent scattering

Unlike X-rays, the neutron scattering length is different even for the same isotope due to the fact that the scattering length depends on the spin state of the isotope and most isotopes have several spin states. This results in an issue about coherent and incoherent scattering for neutrons scattering due to the averaging effects of the scattering lengths (i.e., the difference between $\overline{b^{2}}$ and $\left.(\bar{b})^{2}\right)$. For the system containing 
a large number of nuclei, which is a condition usually well satisfied thinking of one mole of the substance is on the order of $10^{23}$, the measured scattering cross section is approximated to [80],

$$
\frac{d^{2} \sigma}{d \Omega d E}=\frac{1}{2 \pi \hbar} \frac{k^{\prime}}{k} \sum_{j, k} \overline{b_{j} b_{k}} \int_{-\infty}^{\infty}\left\langle e^{-i \vec{Q} \cdot \overrightarrow{r_{k}}(0)} e^{i \vec{Q} \cdot \overrightarrow{r_{j}}(t)}\right\rangle e^{-i \omega t} d t
$$

Where $\overline{b_{j}}$ refers to the average scattering length for the isotope labeled $j$.

The averaging in formula (3.2) can be further expressed by the average value of $b$ $(\bar{b})$ and the average value of $b^{2}\left(\overline{b^{2}}\right)$ as,

$$
\begin{aligned}
\frac{d^{2} \sigma}{d \Omega d E} & =\frac{1}{2 \pi \hbar} \frac{k^{\prime}}{k}\left(\sum_{j, k}(\bar{b})^{2} I_{j k}+\sum_{j}\left(\overline{b^{2}}-(\bar{b})^{2}\right) I_{j j}\right) \\
& =\frac{d^{2} \sigma_{c o h}}{d \Omega d E}+\frac{d^{2} \sigma_{i n c}}{d \Omega d E}
\end{aligned}
$$

where $I_{j k}$ and $I_{j j}$ are shorthand for the integral in formula 3.2. The first term on the right side of the formula (3.3) and (3.4) represents the coherent scattering, and the second term represents the incoherent scattering. For the coherent scattering, the sum is about the atom $j$ located at $r_{j}$ at time $t$ and the atom $k$ located at $r_{k}$ at time $t=0$, and thus it describes interference between the scattered waves from all the nuclei in the sample. On the other hand, the incoherent scattering only involves correlations between the position of the atom $j$ at time zero and the position of itself at time $t$. Thus it is not related with the interference of the scattered waves. In most cases, the incoherent scattering intensity is isotropic and only contributes 
to a structureless background. The information on the atomic structures and the dynamics, however, is all contained in the coherent scattering cross section, $\frac{d^{2} \sigma_{c o h}}{d \Omega d E}$, and the following discussion is mainly about the coherent scattering.

3. General Pair Density Function (PDF) $\rho(\vec{r}, t)$

Formula 3.1 can be rewritten as,

$$
\frac{d^{2} \sigma}{d \Omega d E}=\frac{1}{2 \pi \hbar} \frac{k^{\prime}}{k} N \bar{b}^{2} \int_{-\infty}^{\infty} \rho(\vec{r}, t) e^{i \vec{Q} \cdot \vec{r}} e^{-i \omega t} d \vec{r} d t
$$

and

$$
\rho(\vec{r}, t)=\frac{1}{N} \sum_{j, k} \frac{b_{j} b_{k}}{\vec{b}^{2}} \delta\left(\vec{r}-\left(\overrightarrow{r_{j}}(t)-\overrightarrow{r_{k}}(0)\right)\right)
$$

$\rho(\vec{r}, t)$ is defined as the time-dependent pair density function, and sometimes it is also referred as pair-correlation function. It evaluates the probability of finding two atoms a certain distance apart. From Equation 3.5 and 3.6 it can be seen that what the neutron scattering measured is actually proportional to the time and space Fourier transform of the pair density function.

4. $\operatorname{PDF} \rho(\vec{r}, 0)$ Under the Diffraction

Diffraction is presumably an elastic scattering where $|\vec{k}|=\left|\overrightarrow{k^{\prime}}\right|$ but the neutron diffractometers actually integrate over the energies of scattered neutrons. In formula 3.2 the integral over $\hbar \omega$ gives another delta function $\delta(t)$ and the pair correlation 
function is required to be evaluated at $t=0$. Therefore, formula 3.2 now can be simplified to,

$$
\begin{aligned}
\frac{d \sigma}{d \Omega} & =\frac{1}{N} \sum_{j, k} \overline{b_{j} b_{k}}\left\langle e^{i \vec{Q} \cdot\left(\overrightarrow{r_{j}}-\overrightarrow{r_{k}}\right)}\right\rangle \\
& =\overline{b^{2}}+\frac{1}{N} \sum_{j \neq k} \overline{b_{j} b_{k}}\left\langle e^{i \vec{Q} \cdot\left(\overrightarrow{r_{j}}-\overrightarrow{r_{k}}\right)}\right\rangle \\
& =\overline{b^{2}}+\bar{b}^{2} \int_{-\infty}^{\infty} \rho(\vec{r}, 0) e^{i \vec{Q} \cdot \vec{r}} d \vec{r}
\end{aligned}
$$

where $\rho(\vec{r}, 0)$ describing the pair density of atoms separated by $\vec{r}$ at time, $t=0$.

$$
\rho(\vec{r})=\frac{1}{N} \sum_{j, k} \frac{\overline{b_{j} b_{k}}}{\bar{b}^{2}} \delta\left(\vec{r}-\left(\overrightarrow{r_{j}}-\overrightarrow{r_{k}}\right)\right)
$$

Formula 3.8 indicates as $\vec{Q} \rightarrow \infty, \frac{d \sigma}{d \Omega} \rightarrow \overline{b^{2}}$. A total scattering function, $S(\vec{Q})$, can be defined as (this is Faber-Ziman definition),

$$
S(\vec{Q})=\frac{\frac{d \sigma}{d \Omega}-\bar{b}^{2}+\bar{b}^{2}}{\bar{b}^{2}}
$$

so that when $\vec{Q} \rightarrow \infty, S(\vec{Q}) \rightarrow 1$.

Therefore, the static PDF $\rho(\vec{r})$ is the Fourier transform of the total scattering function $S(\vec{Q})$,

$$
\rho(\vec{r})=\frac{1}{(2 \pi)^{3}} \int_{0}^{\infty} S(\vec{Q}) e^{i \vec{Q} \cdot \vec{r}} d \vec{Q}
$$

Usually the diffraction is measured on the powder samples so $\rho(\vec{r})$ and $S(\vec{Q})$ are isotropic with angular variable $\phi$, and thus the integrations in the equation 3. 12 can be carried out, 


$$
\begin{aligned}
\rho(r) & =\frac{1}{(2 \pi)^{3}} \iiint S(Q) e^{i Q r \cos \theta} Q^{2} d Q d \cos \theta d \phi \\
& =\frac{1}{(2 \pi)^{2}} \int_{0}^{\infty} \int_{-1}^{1} S(Q) e^{i Q r \cos \theta} Q^{2} d Q d \cos \theta \\
& =\frac{1}{2 \pi^{2}} \int_{0}^{\infty} S(Q) \frac{\sin (Q r)}{Q r} Q^{2} d Q \\
& =\frac{1}{2 \pi^{2}} \int_{0}^{\infty} Q S(Q) \frac{\sin (Q r)}{r} d Q
\end{aligned}
$$

Now considering that $S(Q) \rightarrow 1$ at large values of $Q$, the above relation can be further expressed as,

$$
\rho(r)=\rho_{0}+\frac{1}{2 \pi^{2}} \int_{0}^{\infty} Q(S(Q)-1) \frac{\sin (Q r)}{r} d Q
$$

where the constant $\rho_{0}, \rho_{0}=\frac{1}{2 \pi^{2}} \int_{0}^{\infty} \frac{\sin (Q r)}{r} Q d Q$, is the average atomic density.

Based on the equation 3.13 the experimental pair density function, $\rho(r)$, is generated from the measured diffraction data $\frac{d \sigma}{d \Omega}$ or $S(Q)$ (equ. 3.11). Because PDF is obtained by directly Fourier transforming the measured diffraction data, the background intensity should be carefully subtracted. The background intensities are measured on an empty sample container, a vanadium standard and an empty displex [17].

On the other hand, the powder averaging on the equation 3.10 gives rise to,

$$
\rho(r)=\frac{1}{4 \pi r^{2}} \frac{1}{N} \sum_{j, k} \frac{b_{j} b_{k}}{\bar{b}^{2}} \delta\left(r-r_{j k}\right)
$$

which can be used to calculate the PDF for a model where the atom positions are designated based on an assumed crystal symmetry.

From the above discussion it is clear that the experimental PDF is the result of the Fourier transform of the diffraction data, and to obtain the PDF there is no need for any assumption about the crystal structure symmetry. Just because of this the 
PDF is not constrained by any "global" lattice symmetry, and therefore it can provide the information about local lattice distortions in real space. This method, however, is in principle different from the Rietveld refinement [70], which is carried out in the reciprocal space and based on the assumption of a periodic lattice. The fundamental theory about the Rietveld refinement is Bragg scattering and the following is a brief discussion.

\section{Diffraction by a Periodic Lattice- Bragg Scattering}

If material is the form of crystal, the atomic positions are periodic and can be fully described by a crystal symmetry. Under this condition, the general diffraction cross section (equation 3.7) is found to be only related with the atoms in one crystal unit cell, which is the smallest repeatable "block" for the material. Equation 3.15 is the diffraction cross section for crystals, where instead of sum over the total number of atoms in the material (equation 3.7) the summation only takes over the atoms in one crystal unit cell.

$$
\begin{aligned}
\frac{d \sigma}{d \Omega} & =N v_{0}^{*} \delta\left(\vec{Q}-\overrightarrow{\kappa_{h k l}}\right)\left|\sum_{n} b_{n} e^{i \overrightarrow{\kappa_{h k l}} \cdot \overrightarrow{r_{n}}} \cdot e^{-W_{n}}\right|^{2} \\
F_{h k l}(\vec{Q}) & =\sum_{n} b_{n} e^{i \overrightarrow{\kappa_{h k l}} \cdot \overrightarrow{r_{n}}} \cdot e^{-W_{n}}
\end{aligned}
$$

$F_{h k l}(\vec{Q})$ is the nuclear unit-cell structure factor, and it is determined by the type of atoms $\left(b_{n}\right)$ and their locations $\left(\overrightarrow{r_{n}}\right)$ in one unit cell $\left(\overrightarrow{r_{n}}\right.$ is the equilibrium position of the $\mathrm{n}^{\text {th }}$ atom in the unit cell, $v_{0}^{*}$ is the unit cell volume of reciprocal lattice and $N$ is the 
total number of unit cells in the sample). The delta function $\delta\left(\vec{Q}-\overrightarrow{\kappa_{h k l}}\right)$ describes the Bragg's law, i.e., the elastic scattering can only occur when the neutrons' momentum transfer $\vec{Q}$ is equal to the reciprocal lattice constants, $\overrightarrow{\kappa_{h k l}}$. This condition has an equivalent expression in real space and that is $n \lambda=2 d_{h k l} \sin \theta$.

\subsection{Neutron Scattering Instruments}

Neutron scattering instruments can be classified into two major types based on the methods of specifying neutrons' wave vector $\vec{k}$ or speed $\vec{v}$.

This can be realized by the Bragg diffraction discussed in the previous section. In this method, the neutron beam from the source containing different energies are scattered by a monochromator, which is usually a graphite crystal, so at a certain scattering angle, only the neutrons of which energy satisfy the Bragg law are scattered and thus used as incident beams for the sample. Another common method is timeof-flight which is commonly used in the pulse neutron source. The speed of neutron is simply determined by measuring the time taken by a pulse of neutrons to travel a given distance.

- Triple-Axis Spectrometer (TAS)

Triple-axis spectrometers can measure the intensity of scattered neutrons for a particular momentum transfer $\vec{Q}$ and energy transfer $\hbar \omega$. Both incident and scattered neutrons' wave vectors or wavelengths are determined by the method of Bragg 


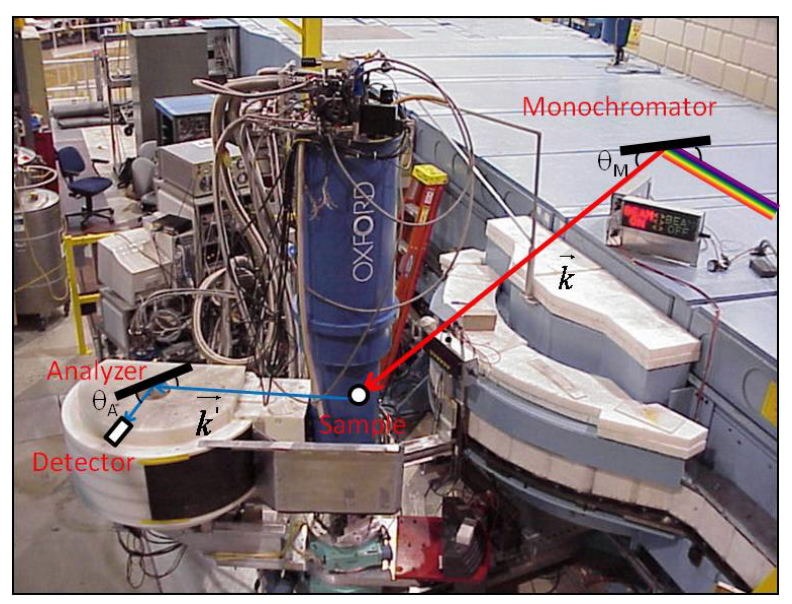

Figure 3.1: The photo of triple axis spectrometer SPINs at NCNR.

diffraction. As an example, Fig. 3.1 shows a photo of the Spin Polarized Inelastic Neutron Spectrometer (SPINS), which is one of the triple-axis spectrometers at NIST Center for Neutron Research. A moderated neutron beam traveling inside the guide hall contains a continuous energy spectrum and thus is indicated as rainbow color in Fig. 3.1. To select the neutrons with a desired energy to be the incident beam (shown as red color), the white beam is incident upon a monochromator, which is usually pyrolitic graphite crystal, so that only the neutrons of which $\vec{k}$ or $\lambda$ match the Bragg's law are scattered at a specific scattering angle $\theta_{M}$. The incident neutrons then scatter from the sample. Determined by what the transfer $\vec{Q}$ and energy transfer $\hbar \omega$ are to be measured for the scattered neutrons, an analyzer which is generally another graphite crystal is placed at a angle $\theta_{A}$ so that only the scattered neutrons with desired $\overrightarrow{k^{\prime}}, \overrightarrow{k^{\prime}}=\vec{k}+\vec{Q}$, (shown as blue color) can be scattered and reach a 


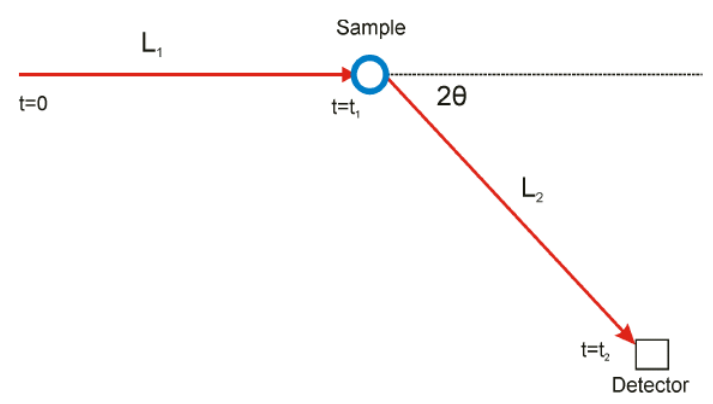

Figure 3.2: Schematic of time-of-flight instrument.

neutron detector.

- Time-of-Flight instruments

In Time-of-Flight instruments, the velocity of neutrons are measured by the time that neutrons take to travel a known distance.

Fig. 3.2 is a general schematic of time-of-flight diffractometer. The time that neutron pulse takes to travel the known distance $L_{1}$ and $L_{2}$ are recorded so the average speed of the pulse can be known by $v=\frac{L}{t}$. From the de Broglie relationship, $k=\frac{m v}{\hbar}$, the wave vector of neutrons is therefore $k=\frac{m L}{\hbar} \frac{1}{t}$. For the diffraction it is assumed that the scattering is elastic and therefore $Q=2 k \sin \theta$, so for the neutrons that are scattered at the angle $2 \theta$ the wave vector transfer $Q$ is $Q=2 k \sin \theta=\frac{2 m L \sin \theta}{\hbar} \cdot \frac{1}{t}$. Thus at a constant scattering angle, $Q$ is inverse proportional to the neutron travel time $t$. On the other hand, as it can be seen that the maximum wave vector transfer, $Q_{\max }$, is proportional to $\sin \theta$. That means for the detector located at a higher scattering angle $2 \theta$, the measured $Q_{\max }$ can reach a larger value. 


\section{Chapter 4}

\section{Sample Preparation}

\subsection{Powder Sample}

The polycrystalline samples were fabricated by conventional solid state reaction. First, the starting materials were weighted in accordance to the stoichiometry molar ratio, and then the mixture was sintered at high temperature to make the solid state reaction happen, and usually the stable high temperature was maintained for one or a few days. After cooling down to the room temperature, however, the resulting compounds may not be a single phase due to the reaction not having been fully completed, so this "baking" process was often repeated several times in order to obtain a final single phase.

The following formula list the starting materials and the synthesis temperature for the compositions of A-site hole doped lanthanum cobaltite $\left(\mathrm{La}_{1-x} \mathrm{Ba}_{x} \mathrm{CoO}_{3}\right.$ and 
$\left.\mathrm{La}_{1-x} \mathrm{Ca}_{x} \mathrm{CoO}_{3}\right)$, B-site magnetic ion doped lanthanum cobaltite $\left(\mathrm{LaCo}_{1-y} \mathrm{Ni}_{y} \mathrm{O}_{3}\right.$ and $\left.\mathrm{LaCo}_{1-y} \mathrm{Fe}_{y} \mathrm{O}_{3}\right)$, and praseodymium cobaltite $\left(\mathrm{PrCoO}_{3}\right.$, and the reference compound $\left.\mathrm{PrAlO}_{3}\right)$

$$
\begin{aligned}
& \frac{1-x}{2} \mathrm{La}_{2} \mathrm{O}_{3}+\frac{1}{3} \mathrm{Co}_{3} \mathrm{O}_{4}+x \mathrm{BaCO}_{3}+\frac{1+3 x}{12} \mathrm{O}_{2} \stackrel{1200^{\circ} C}{\longrightarrow} \mathrm{La}_{1-x} \mathrm{Ba}_{x} \mathrm{CoO}_{3}+x \mathrm{CO}_{2} \\
& \frac{1-x}{2} \mathrm{La}_{2} \mathrm{O}_{3}+\frac{1}{3} \mathrm{Co}_{3} \mathrm{O}_{4}+x \mathrm{CaCO}_{3}+\frac{1+3 x}{12} \mathrm{O}_{2} \stackrel{1200^{\circ} C}{\longrightarrow} \mathrm{La}_{1-x} \mathrm{Ca}_{x} \mathrm{CoO}_{3}+x \mathrm{CO}_{2} \\
& \frac{1}{2} \mathrm{La}_{2} \mathrm{O}_{3}+\frac{1-y}{3} \mathrm{Co}_{3} \mathrm{O}_{4}+y \mathrm{NiO}+\frac{1+2 y}{12} \mathrm{O}_{2} \stackrel{1200^{\circ} C}{\longrightarrow} \mathrm{LaCo}_{1-y} \mathrm{Ni}_{y} \mathrm{O}_{3} \\
& \frac{1}{2} \mathrm{La}_{2} \mathrm{O}_{3}+\frac{1-y}{3} \mathrm{Co}_{3} \mathrm{O}_{4}+\frac{y}{2} \mathrm{Fe}_{2} \mathrm{O}_{3}+\frac{1-y}{12} \mathrm{O}_{2} \stackrel{1200^{\circ} C}{\longrightarrow} \mathrm{LaCo}_{1-y} \mathrm{Fe}_{y} \mathrm{O}_{3} \\
& \frac{1}{6} \mathrm{Pr}_{6} \mathrm{O}_{11}+\frac{1}{3} \mathrm{Co}_{3} \mathrm{O}_{4} \stackrel{1200^{\circ} C}{\longrightarrow} \mathrm{PrCoO}_{3}+\frac{1}{12} \mathrm{O}_{2} \\
& \frac{1}{6} \mathrm{Pr}_{6} \mathrm{O}_{11}+\frac{1}{2} \mathrm{Al}_{2} \mathrm{O}_{3} \stackrel{1350^{\circ} C}{\longrightarrow} \operatorname{PrAlO}_{3}+\frac{1}{6} \mathrm{O}_{2}
\end{aligned}
$$

Note that when exposed to the moist air at room temperature, lanthanum oxide easily forms a hydrated oxide with a large volume increase [59] (you can actually check this by leaving some lanthanum oxide in the open air for a few days and you will notice the volume expands a lot), before weighting the starting materials, $\mathrm{La}_{2} \mathrm{O}_{3}$ should be preheated for dehydration, $2 \mathrm{La}(\mathrm{OH})_{3}+$ heat $\rightarrow \mathrm{La}_{2} \mathrm{O}_{3}+3 \mathrm{H}_{2} \mathrm{O}$. Usually we heat the compound at $600{ }^{\circ} \mathrm{C}$ for about $10 \mathrm{hrs}$.

\subsection{Sample Quality Examination}

After a powder sample is made, phase identification and single phase determination was performed using X-ray powder diffraction measurements in our lab. The target is $\mathrm{Cu}$, and $\mathrm{K}-\alpha$ radiation is used with a wavelength of $1.5418 \AA$. The bulk magnetization and resistivity are measured using a Quantum Design Physical Property 
Measurement System (PPMS) in our lab. The $d c$ mode magnetization as a function of temperature $M(T)$ and magnetic field dependence $M(H)$ were measured using the ACMS option, and the $d c$ resistivity measurements were made using a standard four-probe technique.

\subsection{Single Crystal Sample}

For neutron scattering measurements, high-quality and large volume crystals become indispensable. In the case of oxides, so far the most successful technique for producing large crystals with high purity is the floating-zone method using an image furnace.

- Image Furnace \& Floating Zone method

The image furnaces can be two-mirror or four-mirror systems. Fig. 4.1 shows a schematic of the two-mirror furnace and Fig. 4.2 is a photo of the two-mirror furnace in our lab.

A halogen lamp is located at one of the two focal points of the semi-ellipsoidal mirror, so the emitted lights will be focused at the other focal point forming a heated zone. Compared with a 2-mirror furnace, a 4-mirror furnace heats this zone in a more uniform manner, but at the same time, the difficulty to align the two focal points of the two pairs of ellipsoidal mirrors to exactly overlap with each other makes the loss of focusing a problem for a 4-mirror furnace. 


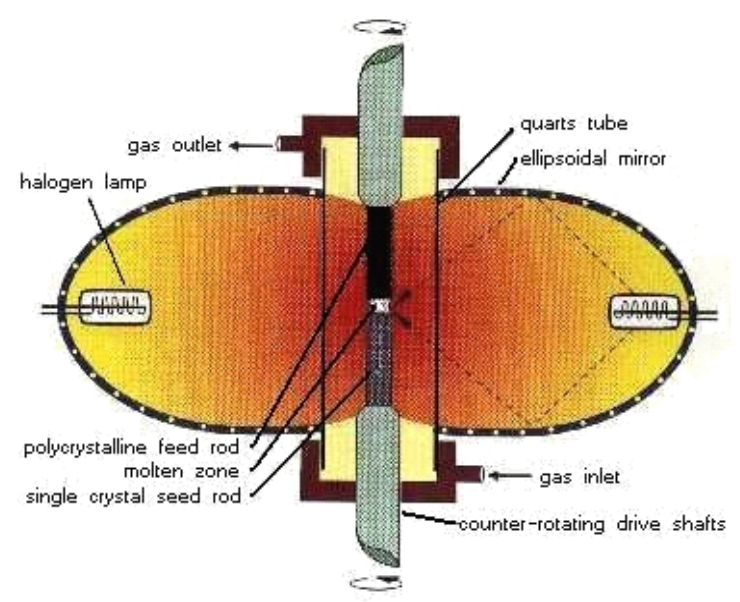

Figure 4.1: Schematic of a two-mirror image furnace, obtained from ref [22]

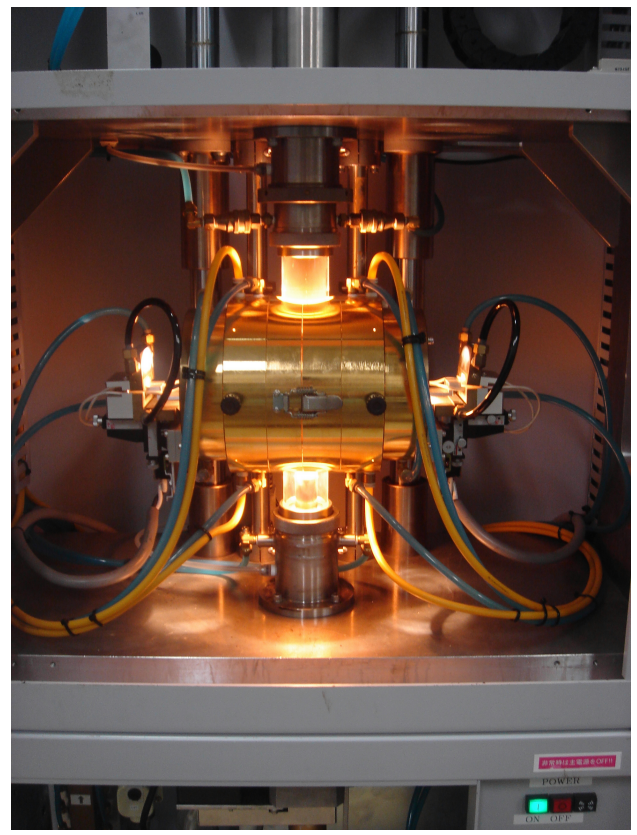

Figure 4.2: Photo of the two-mirror image furnace 
Before starting the crystal growth, the feed rod and seed rod need to be prepared. The feed rod is made from the single phase powder compound. The rod shape is obtained by the following procedure: first the powder compounds are loaded into a rubber tube with diameter $\varnothing \sim 8 \mathrm{~mm}$ and the length $\mathrm{h} \sim 10 \mathrm{~cm}$. Before sealing the tube, the inside air is evacuated. The sealed tube is then hydrostatic pressed to form a compact cylindrical shape. The empirical pressure that we found to be suitable for the cobaltite materials, meaning not too high to cause an internal breaking of the rod or not too low to form a compact rod, is $\sim 50 \mathrm{MPa}$. The rod is then sintered in air for two to three days at a temperature that is slightly higher than the synthesis temperature, which in our case is between 1200 to $1250^{\circ} \mathrm{C}$. The seed rod is a grown single crystal.

In the floating-zone method, the two rods are aligned along the same axis (Fig. 4.1, the upper one is the feed rod and the lower one is the seed rod). The ends of the two rods are heated to melt, and then after they are joined together, a molten zone formed by the melt liquid is held in place by its own surface tension. This makes the floating zone method advantageous because single crystals can be grown without contamination from the use of a container. Two counter-rotating shafts are used to support the two rods, and the rotation of the shafts helps to stabilize and mix the molten material and also produces a uniform heating. The molten zone is slowly translated along the rod by the vertical movement of either the mirror assembly or two-rod assembly. The new crystal is precipitated after the molten liquid is cooled 
down and solidified.

- Growth Conditions

The growing conditions such as the applied voltage on the lamps, the growth speed, the rotation speed of the feed and seed rods, and the gas atmospheres, etc., can vary significantly for different samples. As for $\mathrm{La}_{1-x} \mathrm{Ba}_{x} \mathrm{CoO}_{3}$ and $\mathrm{LaCo}_{1-y} \mathrm{Ni}_{y} \mathrm{O}_{3}$, empirically, we found the applied voltage of a stable condition for growing $\mathrm{LaCo}_{1-y} \mathrm{Ni}_{y} \mathrm{O}_{3}$ is higher than the one for $\mathrm{La}_{1-x} \mathrm{Ba}_{x} \mathrm{CoO}_{3}$, and when the $\mathrm{Ba}$ concentration increases the applied voltage to maintain a stable growth should be lowered. This is most likely because $\mathrm{Ba}$ doping lowers the melting point of the compounds. Note that oxygen is needed for forming the doped compounds as indicated in the reaction formula in Chap 4.1, the growths were conducted under the $\mathrm{O}_{2}$ flow to minimize the possibility of oxygen vacancies.

For the compositions of $\mathrm{LaCo}_{1-y} \mathrm{Ni}_{y} \mathrm{O}_{3}(y=0.005,0.01,0.10)$ and $\mathrm{La}_{1-x} \mathrm{Ba}_{x} \mathrm{CoO}_{3}$ $(x=0.03,0.06,0.10,0.15$ and 0.18$)$, large volume single crystal samples with high quality were successfully grown. Fig. 4.3 shows an example of a grown single crystal of $\mathrm{LaCo}_{0.9} \mathrm{Ni}_{0.1} \mathrm{O}_{3}$. However, for high concentration Ba samples, $\mathrm{La}_{1-x} \mathrm{Ba}_{x} \mathrm{CoO}_{3}(x>$ $18 \%$ ), we found that the higher the doping level, the more difficult it was to keep a stable growth condition due to two main issues. In the following, the issues will be discussed along with some useful methods that we found by trial and error.

(1) From the experiments, we noticed that for the compositions with high Ba concentrations, the feed rods tended to be fragile and often cracked during the growing 


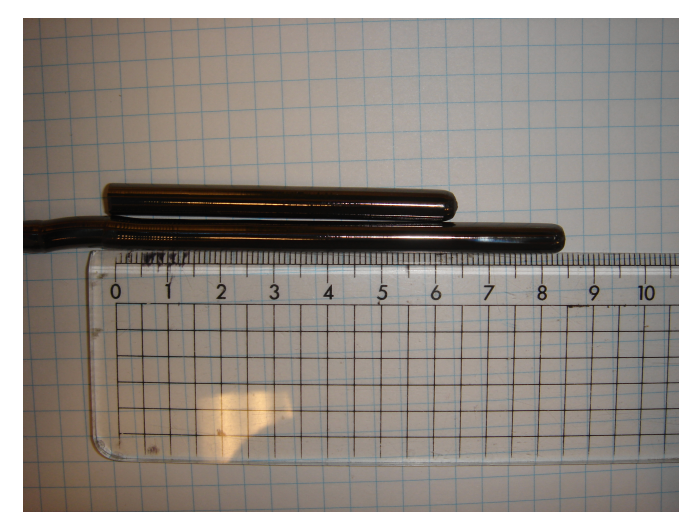

Figure 4.3: Photo of grown single crystal for $\mathrm{LaCo}_{0.9} \mathrm{Ni}_{0.1} \mathrm{O}_{3}$.

when the temperature rose. Considering that the same condition and methods were applied to prepare all the feed rods and the same image furnace was used for growing, the tendency of being fragile might be intrinsic for the feed rod of high Ba doped compositions. Notice from the right side of the reaction formula for Ba compounds (Chap 4.1) that the production of compositions with higher Ba concentrations was also accompanied by a larger amount of carbon dioxide, $\mathrm{CO}_{2}$, which means the reaction for higher Ba compounds releases more gas. This may result in the compound being more porous and may cause the fragility and cracking. In order to increase the density of the feed rod, a 'fast-growth' technique was used. In this method, the feed rod is melted and solidified as it is grown but at a much higher speed, $\sim 20 \mathrm{~mm} / \mathrm{hr}$, than the growth speed which typically is $\sim 1-2 \mathrm{~mm} / \mathrm{hr}$. After the fast-growth, the density of the feed rod is found to increase dramatically and the 'cracking' problem during the growing is solved. 
(2) The higher the Ba doping, the more unstable the melting zone. From the experiments, we observed a puzzling situation where the melting zone all of sudden collapsed while the growing appeared stable and the melting zone was in a good shape and size. The reason for this "sudden death" could be because the viscosity of molten liquid is lowered when Ba concentration increased so that the surface tension is not sufficient to hold the molten zone. To stabilize the molten zone we applied a pressurized $\mathrm{O}_{2}$ atmosphere at 1 atm. By this way the growing process was prolonged, but still after $\sim 10$ hrs the molten zone ended up collapsed. Thus, in the future attempts higher $\mathrm{O}_{2}$ pressure might be worth try. Besides, noble gas such as Ar flow was also tried, but it appeared that the single crystal was not able to form in this atmosphere, and the grown product was still polycrystalline or amorphous. 


\title{
Chapter 5
}

\section{Magnetic Phase Separation and Its}

\author{
Trend in $\mathbf{L a}_{1-x} \mathbf{A}_{x} \mathbf{C o O}_{3}\left(\mathbf{A}=\mathbf{C a}^{2+}\right.$, \\ $\mathrm{Sr}^{2+}$ or $\left.\mathrm{Ba}^{2+}\right)$
}

\subsection{Global Magnetic State with Hole Doping}

Holes are introduced into the system by the substitution of $\mathrm{La}^{3+}$ ions with divalent ions of $\mathrm{Ca}^{2+}, \mathrm{Sr}^{2+}$ or $\mathrm{Ba}^{2+}$. It is found that doping will lead the system, $\mathrm{La}_{1-x} \mathrm{~A}_{x} \mathrm{CoO}_{3}$, to a FM state with all three dopants. Fig. 5.1 is a comparison of the magnetic phase diagrams for $\mathrm{Ca}^{2+}, \mathrm{Sr}^{2+}$ and $\mathrm{Ba}^{2+}$ doping [44]. It can be seen that all three compositions become FM after doping, but $\mathrm{La}_{1-x} \mathrm{Ca}_{x} \mathrm{CoO}_{3}$ becomes FM right after doping, while for Sr and Ba dopings, the systems first enter a spin-glass (SG) state, 


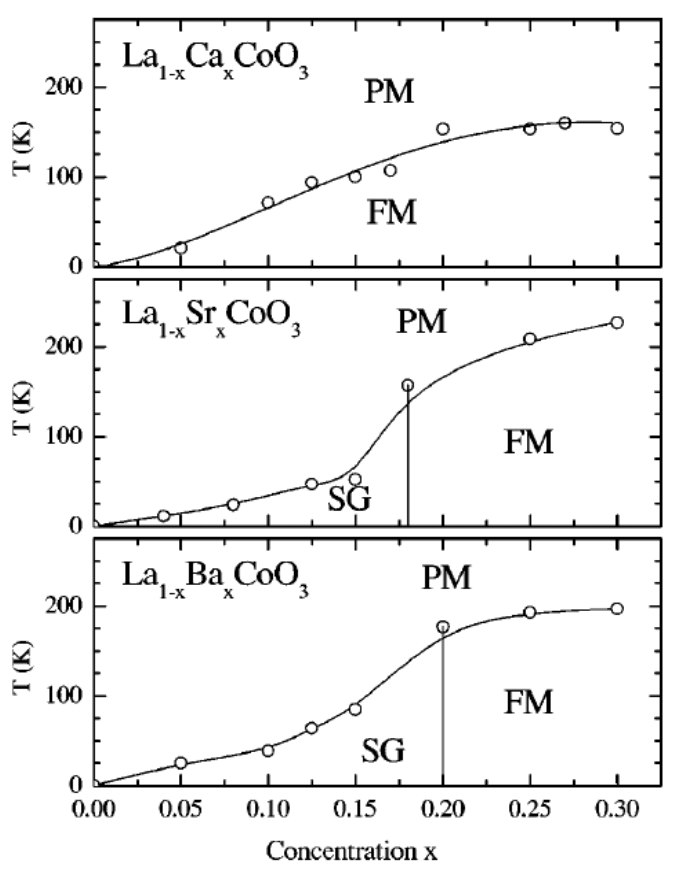

Figure 5.1: The magnetic phase diagrams for $\mathrm{Ca}, \mathrm{Sr}$ and $\mathrm{Ba}$ doping. The figure is from ref [44].

then at $x \sim 18 \%$ in $\mathrm{Sr}$ and $x \sim 20 \%$ in Ba the systems develop to the FM state.

In the previous studies on $\mathrm{La}_{1-x} \mathrm{Sr}_{x} \mathrm{CoO}_{3}$ the bulk susceptibility and neutron scattering measurements $[49,63,44]$ indicated the microscopic nature of its SG state to be a "cluster glass" state (refer to Chap 2.3). For $\mathrm{La}_{1-x} \mathrm{Ba}_{x} \mathrm{CoO}_{3}$ similar bulk susceptibility with doping and temperature was observed in the SG state. Fig. 5.2 shows the data for the single crystal samples of $\mathrm{La}_{1-x} \mathrm{Ba}_{x} \mathrm{CoO}_{3}(x=0.03$ to 0.18$)$. For the composition with the lowest doping (3\%), both the zero-field-cooled and field-cooled data follow a Curie-Weiss-like curve. No magnetic transition or spin-state transition is observed. For the rest of the compositions with higher doping levels, a bifurcation clearly occurred between the ZFC and FC curves, which is a typical feature for the 


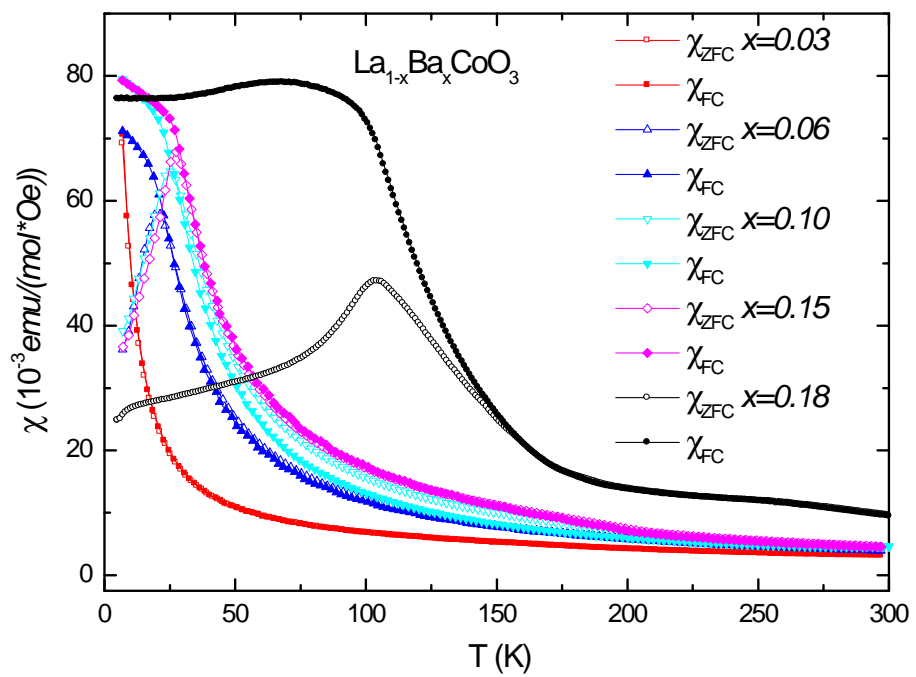

Figure 5.2: Bulk magnetic susceptibilities for single crystals of $\mathrm{La}_{1-x} \mathrm{Ba}_{x} \mathrm{CoO}_{3}$ $(x=0.03$ to 0.18$)$ as a function of temperature.

spin-glass state. Moreover, a cusp in the ZFC data started to barely show up for $15 \%$ sample, and for $18 \%$ sample it became very obvious. This cusp in the ZFC magnetization was also observed in the Sr doped samples but at lower concentrations, such as $15 \%$ and $10 \%$, and its was suggested to be caused by a competition between the random orientation of short-range-ordered clusters and the external field [90]. The occurrence of the cusp in the Ba samples at the higher doping levels thus indicated that the SG state was maintained up to a higher concentration. In other word, the SG-FM transition occurred at a higher doping level in Ba than in Sr. Recall Cadoping quickly enters the FM state. The FM transition, therefore, showed a strong dependence on the type of dopant. Why? 


\subsection{Coupled FM-IMT and the Magnetic Interac- tion Mechanism}

Hole doping not only drives the system to be a ferromagnet but also a metal. More importantly, FM and insulator-metal transitions (IMT) happen simultaneously. A microscopic mechanism called "double exchange (DE)" is generally applied to understand the coupled FM-IMT in the hole doped systems [49, 14, 90, 44].

DE was first proposed by Zener [99] to understand the coupled correlation between electrical conduction and ferromagnetism in hole doped manganites, $\mathrm{La}_{1-x} \mathrm{~A}_{x} \mathrm{MnO}_{3}$. This mechanism is applied very well in hole doped cobaltites also: recall the perovskite structure where $\mathrm{Co}^{3+}$ is located at each corner of the psudo-cubic cell, and in between the closest $\mathrm{Co}^{3+}$ ions there is an $\mathrm{O}^{2-}$ ion. It can be seen that the $d$ orbitals of $\mathrm{Co}^{3+}$ are not extended enough to have a direct overlap with another $\mathrm{Co}^{3+}$, but possibly overlap with the $p$ orbitals of $\mathrm{O}^{2-}$ ion. As a result, a hybridization of $p-d$ orbitals can introduce an exchange interaction. Fig 5.3 shows a schematic for the FM interaction via $\mathrm{Co}^{3+} \mathrm{O}^{2-}-\mathrm{Co}^{4+}$. Based on the neutron scattering experiments and theoretical calculation, the spin state of $\mathrm{Co}^{3+}$ ions in hole doped systems is stabilized in the IS state[49, 50, 63, 64, 43] (discussed in Chap 2.4), so its $e_{g}$ electron can hop to the empty $e_{g}$ orbitals of its nearest neighbor, most likely a $\mathrm{Co}^{4+}$ ion. By doing so, the system's kinetic energy is lowered. This hopping is only energetically favorable when the core spins of both donating and receiving magnetic ions are FM-aligned. At the 


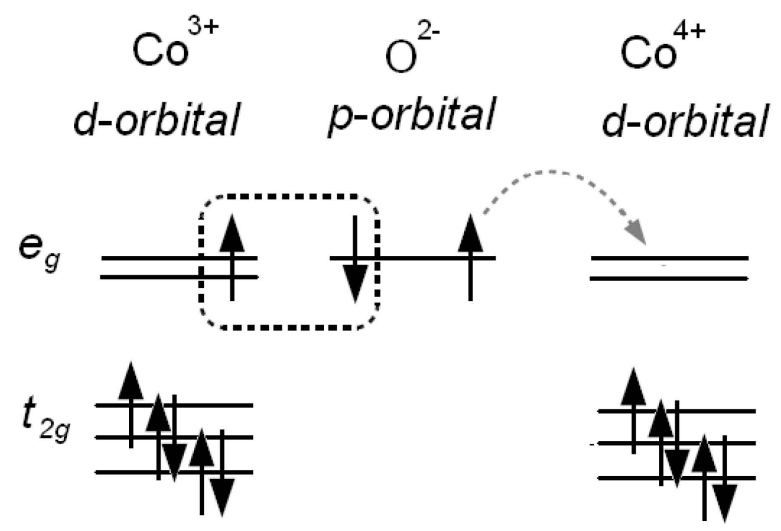

Figure 5.3: Double exchange between an IS $\mathrm{Co}^{3+}$ and a $\mathrm{LS} \mathrm{Co}^{4+}$ ion.

same time, the spins of $e_{g}$ and $t_{2 g}$ orbitals of the same atom are co-aligned by Hund's coupling. As a result, $\mathrm{Co}^{3+}$ and $\mathrm{Co}^{4+}$ ions are $\mathrm{FM}$ coupled. The hopping of the electrons, on the other hand, contributes to the electrical conductivity.

This first happens among a few Co sites, forming a FM-metallic cluster. As the doping level increases, the amount of $\mathrm{Co}^{4+}$ ions increases, which could result in the growth of both the size and the number of these clusters. At some point (it is usually referred to the percolation threshold), these FM-metallic clusters are able to connect to each other and establish a long-range FM and conductive path. In a macroscopic scale, the compound becomes a FM metal.

If the two magnetic ions have an identical electronic configuration, however, the indirect exchange will lead to an AFM interaction rather than a FM one, i.e. an AFM super-exchange. Fig. 5.4 shows a schematic picture for the AFM coupling between two IS $\mathrm{Co}^{3+}$ ions. Because the Pauli exclusion principle prevents two electrons in 


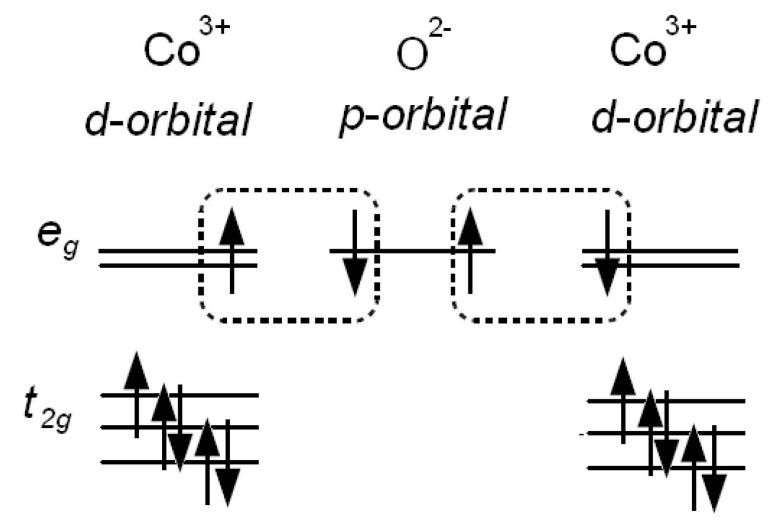

Figure 5.4: AFM super-exchange between two IS $\mathrm{Co}^{3+}$ ions.

the same orbital to have the same state, the spin in the $e_{g}$ orbital of the $\mathrm{Co}^{3+}$ ion is AFM coupled with the spin of $\mathrm{O}^{2-}$. The same coupling happens between the other spin of $\mathrm{O}^{2-}$ with the neighboring $\mathrm{Co}^{3+}$ ion on the other side. As a result, two $\mathrm{Co}^{3+}$ ions are AFM coupled to each other. This coupling does not involve the hopping of electrons so that the $e_{g}$ electrons are localized at the same site; in other word, this AFM exchange is associated with an insulating state.

In the above discussion, the DE actually refers to the FM super exchange between two mixed-valence ions of the same species, such as $\mathrm{Co}^{3+}-\mathrm{O}^{2-}-\mathrm{Co}^{4+}$. However, a FM super exchange between two different species magnetic ions is also possible. For example, doping $\mathrm{Ni}$ ions also leads the system $\mathrm{LaCo}_{1-y} \mathrm{Ni}_{y} \mathrm{O}_{3}$ to the $\mathrm{SG}$ and FM state. In order to understand the occurrence of the ferromagnetism due to Ni doping, a FM correlation via super-exchange of $\mathrm{Co}^{3+}-\mathrm{O}^{2-}-\mathrm{Ni}^{3+}$ was proposed [60]. 


\subsection{Magnetic Phase Separation Trends with A-site}

\section{Dopant}

Noticing the ionic sizes increase significantly from $\mathrm{Ca}^{2+}$ to $\mathrm{Sr}^{2+}$ to $\mathrm{Ba}^{2+}$, the evolution of the magnetic phases with different dopants (shown in Fig. 5.1) seems to imply some kind of trend, where the size of the dopant appears to be related to the development of the SG phase: for the smallest dopant, $\mathrm{Ca}^{2+}$, the SG is absent; as the size of dopant increases to that of the $\mathrm{Sr}^{2+}$ ion, the SG state appears below $x \sim$ $18 \%$; for the largest dopant, $\mathrm{Ba}^{2+}$, the $\mathrm{SG}$ phase extends to a higher concentration, $x \sim 20 \%$. It appeared that the large dopant favors the presence of the SG state.

One may wonder what drives this trend? what the microscopic mechanism is behind this trend? Thinking that since a coexistence of the FM and IC regions was identified in the SG state of $\mathrm{La}_{1-x} \mathrm{Sr}_{x} \mathrm{CoO}_{3}$, a thorough investigation across all three different dopants may help to show the answer. Therefore, the elastic neutron scattering measurements were carried out on the single crystals of $\mathrm{La}_{1-x} \mathrm{Ca}_{x} \mathrm{CoO}_{3}$ and $\mathrm{La}_{1-x} \mathrm{Ca}_{x} \mathrm{CoO}_{3}$ [95]. The measurements were carried out using the cold triple-axis spectrometer SPINS at the NIST Center for Neutron Research with a fixed $E_{f}=3.6$ meV, for $\mathrm{La}_{0.82} \mathrm{Ba}_{0.18} \mathrm{CoO}_{3}$ it was measured on the thermal spectrometer TOPAN of Tohoku University at the JRR-3 reactor of the Japan Atomic Energy Agency with a fixed $E_{f}=13.5 \mathrm{meV}$, and for $\mathrm{La}_{0.9} \mathrm{Ba}_{0.1} \mathrm{CoO}_{3}$ it was measured on both SPINS and the HB-1 spectrometer with a fixed $E_{f}=14.7 \mathrm{meV}$ at the High Flux Isotope Reactor 


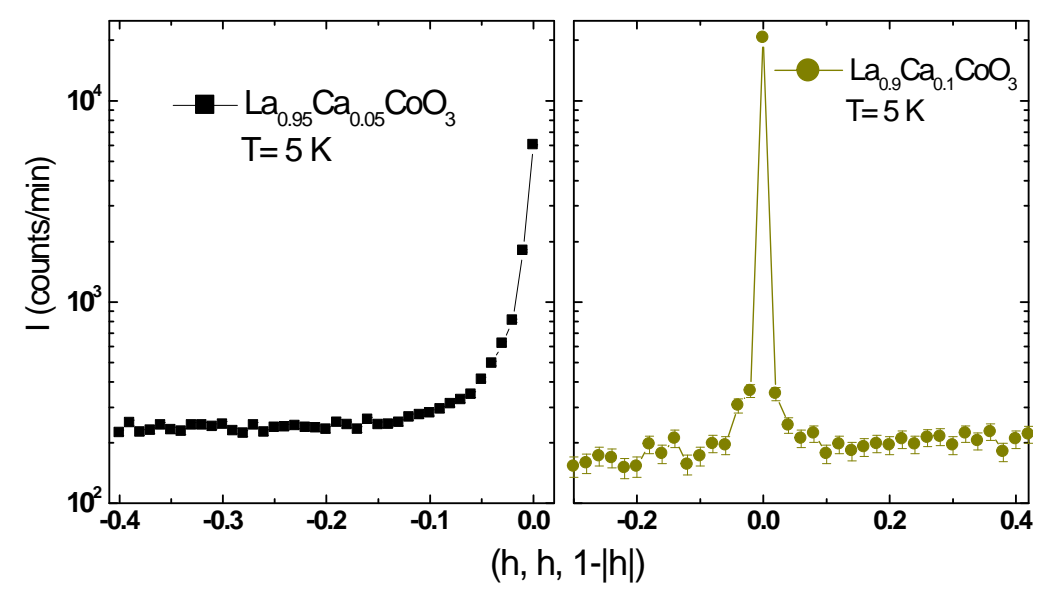

Figure 5.5: The diagonal scans along [111] around the Bragg peak of (001) in single crystals of $\mathrm{La}_{0.95} \mathrm{Ca}_{0.05} \mathrm{CoO}_{3}$ and $\mathrm{La}_{0.9} \mathrm{Ca}_{0.1} \mathrm{CoO}_{3}$. No magnetic satellite peaks have been observed.

of the Oak Ridge National Laboratory. The pseudocubic notation is used to analyze the data and the elastic intensity, indicative of static correlations in the time scale of the neutron experiment, was measured in the (hhl) plane.

For Ca doping, only FM scattering was observed, and no magnetic super lattice was ever found. This is consistent with its magnetic phase where doping quickly brings the system into the FM state. Fig. 5.5 shows the scans along the diagonal directions, [111], around the Bragg peak of (001) for single crystals of $\mathrm{La}_{0.95} \mathrm{Ca}_{0.05} \mathrm{CoO}_{3}$ and $\mathrm{La}_{0.9} \mathrm{Ca}_{0.1} \mathrm{CoO}_{3}$ at $5 \mathrm{~K}$. Compared with the super-lattice magnetic peaks observed in this direction in $\mathrm{Sr}$ samples, there are no satellite magnetic peaks probed in $\mathrm{Ca}$ samples. On the other hand, the FM scattering intensities appear much stronger than Sr sample at the same concentration, for example, in $10 \%$ doped sample, the FM scattering in Sr sample appeared as broad intensity around the Bragg peaks 
indicating the finite size of the FM correlations [62], but in $\mathrm{La}_{0.9} \mathrm{Ca}_{0.1} \mathrm{CoO}_{3}$, the shape of the peak at (001) (left panel of Fig. 5.5) is already Bragg-like, indicating the FM correlation became considerably more long-range ordered.

For Ba doping, however, the magnetic superlattice peaks appear again in the SG phase with a similar pattern as the one in Sr sample. Fig. 5.6 is an elastic scattering contour of $(h h l)$ plane in reciprocal space for the single crystal of $\mathrm{La}_{0.9} \mathrm{Ba}_{0.1} \mathrm{CoO}_{3}$ at $5 \mathrm{~K}$. The inset in the right upper corner plots the contour of $(h h l)$ plane for the single crystal of $\mathrm{La}_{0.9} \mathrm{Sr}_{0.1} \mathrm{CoO}_{3}$. Clearly four broad satellite peaks are located around the center (001) Bragg peak along the diagonal directions, and the Bragg peak is broadened due to the short-range FM correlations. Besides the similar patterns, however, the magnetic superlattice scatterings appear more prominent for the Ba compositions than the ones in the $\mathrm{Sr}$ at the same concentration.

To investigate the dependence of the magnetic scattering signals as a function of the doping concentration, the diagonal scans across magnetic satellite peaks were measured for the compositions with $x=0.03$ up to 0.18 . Fig. 5.7 (a) is the diagonal cut for $\mathrm{La}_{1-x} \mathrm{Ba}_{x} \mathrm{CoO}_{3}(x=0.18)$ along the (111) direction through the (111) Bragg peak, and (b) to (d) are the cuts along (111) and (11-1) through the (001) Bragg peak for $\mathrm{La}_{1-x} \mathrm{Ba}_{x} \mathrm{CoO}_{3}$ at the concentrations of $x=0.15,0.10,0.03$, respectively. The data shown in black are at low temperature (below the magnetic transition) and red at high temperature. First, notice that the satellite peaks and the broadened intensity around the Bragg peak correspond to the magnetic signal because they appear at low 


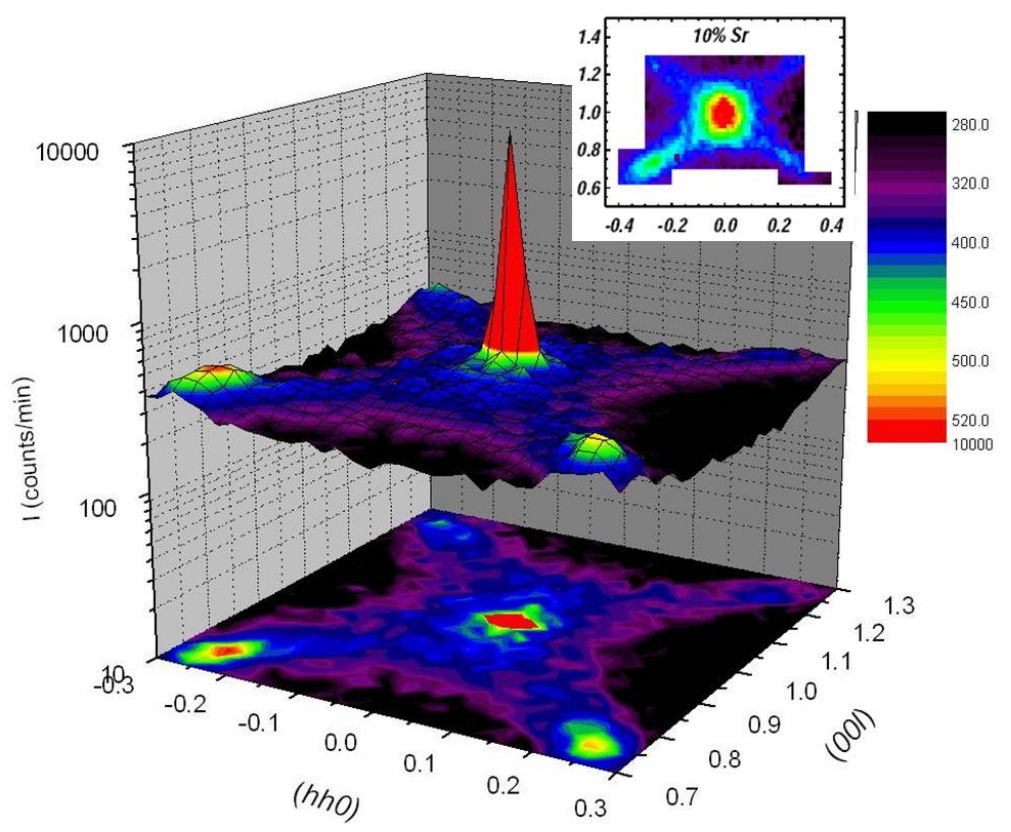

Figure 5.6: The elastic neutron scattering contour in (hhl) plane around (001) position in the single crystal of $\mathrm{La}_{0.9} \mathrm{Ba}_{0.1} \mathrm{CoO}_{3}$ at $5 \mathrm{~K}$. The inset at the right upper corner is the contour for the $\mathrm{Sr}$ composition at the same doping concentration, $\mathrm{La}_{0.9} \mathrm{Sr}_{0.1} \mathrm{CoO}_{3}$. The magnetic super lattice scatterings appear more obvious in Ba sample than the ones in Sr. 
(h, h, h)

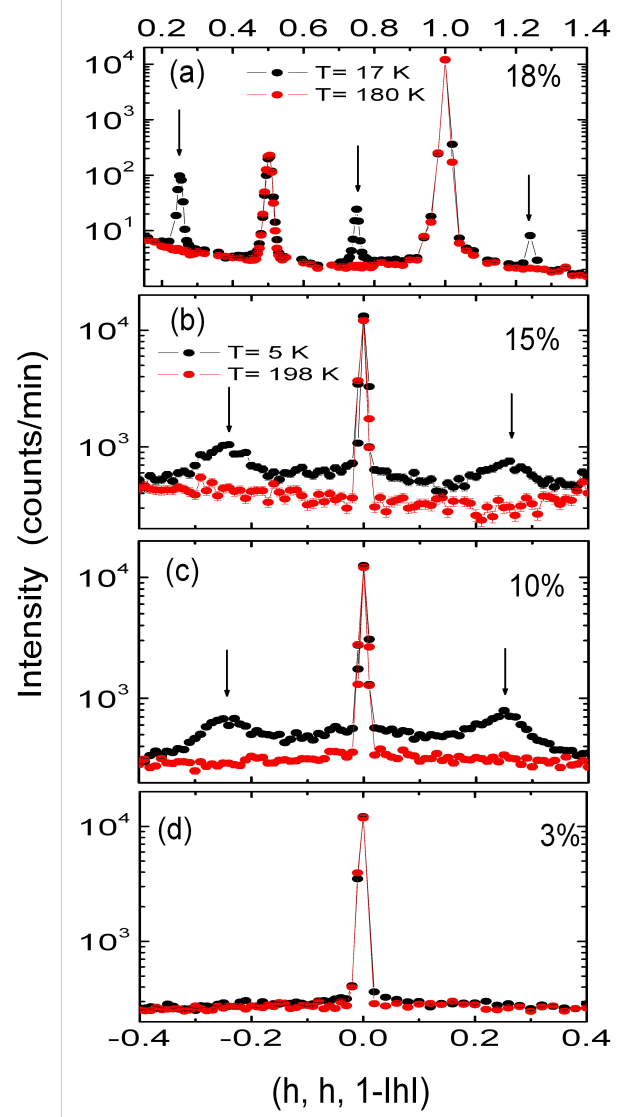

Temperature (K)

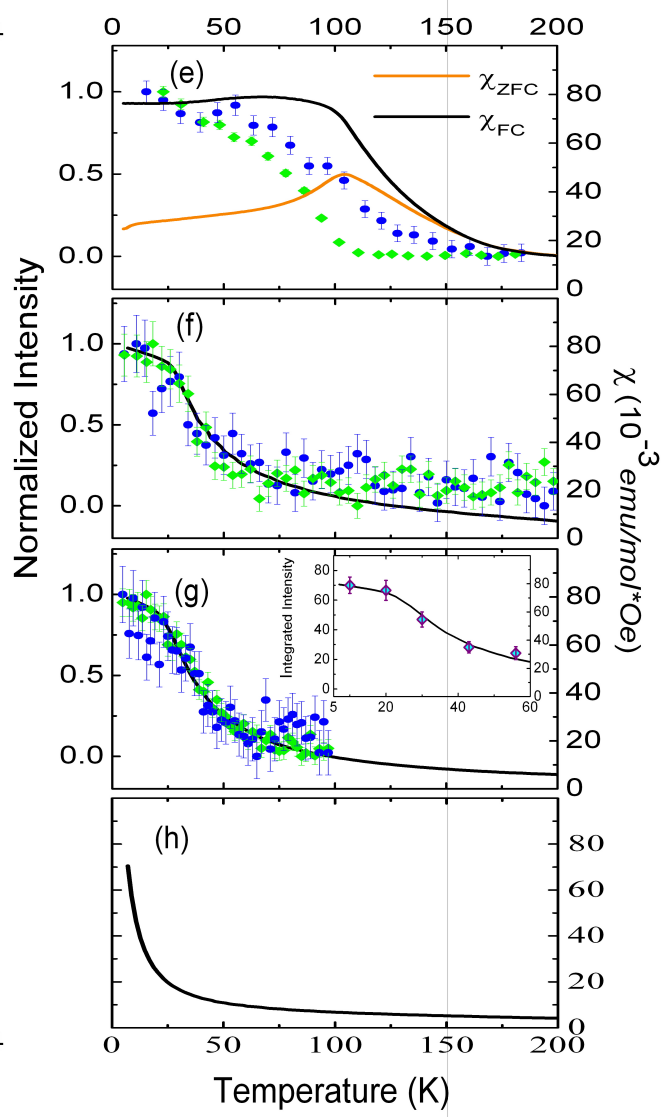

Figure 5.7: (a)-(d): the diagonal scans below (black) and above (red) the magnetic transition temperature for $\mathrm{La}_{1-x} \mathrm{Ba}_{x} \mathrm{CoO}_{3}(x=0.18,0.15,0.10,0.03)$. (e)-(f) the compounds' corresponding order parameters, the FM (blue) and the satellite (green), compared with the bulk magnetic susceptibility, field-cool (black) and zero-field-cool (orange). The inset of (g) is the comparison of the integrated satellite intensity with $\chi_{\text {bulk }}$. 
temperature but disappear above the transition temperature. The magnetic intensity at the Bragg peak position corresponds to the FM intensity and the broadening feature indicates the FM correlation is short-range order. The satellite peaks corresponds to the second magnetic correlation and if the peak position is not commensurate with the lattice periodicity, it is related with an incommensurate correlation. Second, it can be seen that the magnetic scattering intensities of both FM and satellite peaks visibly increase as the amount of doping increases. For the $3 \%$ doped compound, neither magnetic superlattice nor FM scattering intensities have been observed, then as the amount of doping increases both intensities show up as broad intensities, and for $18 \%$ doped sample, both magnetic intensities become Bragg-like. This indicates that the ranges of both FM and AFM orders gradually grow in size.

The measurements on the order parameters of the two magnetic signals further indicate a corresponding relationship with the bulk magnetic susceptibility. Fig. 5.7 (e) to (h) show the order parameters of FM (blue) and satellite peaks (green) compared with the bulk magnetic susceptibility data (black line). For the $3 \%$ sample shown in (h), consistent with the paramagnetic behavior suggested by the bulk susceptibility, there is no magnetic signal in the elastic scattering channel. For $10 \%$ and $15 \%$ samples, (f) and (g), the order parameters of both FM and satellite peaks overlap each other, and at the same time coincide with the bulk susceptibility. The inset of $(\mathrm{g})$ is the integrated intensity of the satellite peaks for the $10 \%$ sample, which is proportional to the volume fraction for the $x=0.10$. Again, the integrated intensity also 


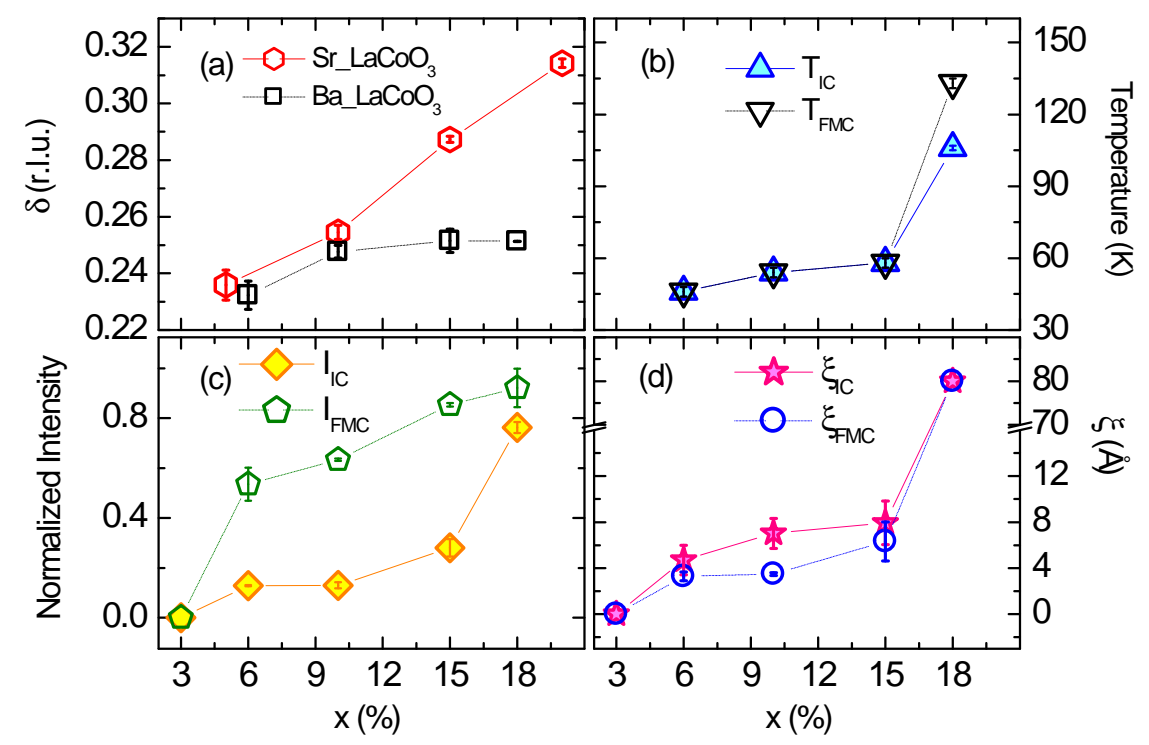

Figure 5.8: Data for the Ba crystals as a function of hole concentration. (a) is a plot of the IC peak position, $\delta$, with $x \%$ which is compared to the dependence on $x \%$ obtained previously for Sr crystals. (b) is a plot of the order parameter for the two magnetic phases. (c) is a plot of the normalized satellite peak intensity, $I_{I C}$ and the (001) Bragg peak, $I_{F M}$. (d) is a plot of a comparison of the correlation lengths for the commensurate, $\xi_{F M}$ and incommensurate, $\xi_{I C}$. Note that $\xi$ is comparable in the two magnetic phases at all $x$, while the value of $80 \AA$ for the $18 \%$ is a lower bound since in the experimental setup of TOPAN, the peaks became resolution limited.

compares well with bulk susceptibility. For $18 \%$ sample, shown in (e), however, the order parameter of FM is different than the satellite: FM appears to order at a higher temperature than the satellite peaks. On the other hand, this is the concentration that shows an obvious cusp in the zero-field-cooled data of $\chi$. The cusp appears at about the same temperature as the transition temperature of the satellite signals.

Based on Fig. 5.7, Fig. 5.8 summarized several important parameters as following. In panel (a) the incommensuratibility, $\delta$, are compared in $\mathrm{Sr}$ and $\mathrm{Ba}$ compounds. The 
satellite peaks locate at $(h \pm \delta, k \pm \delta, l \pm \delta)$ and for Sr doped compounds $\delta$ are not commensurate with the periodicity of the lattice constant. However, after the dopant changes from $\mathrm{Sr}^{2+}$ to the bigger ion, $\mathrm{Ba}^{2+}$, the satellite peaks become commensurate at the position of $\left(\frac{1}{4}, \frac{1}{4}, \frac{1}{4}\right)$ in cubic notation, which corresponds to the $\left(\frac{1}{2}, \frac{1}{2}, \frac{1}{2}\right)$ position in rhombohedral cell. In other words, the magnetic IC satellite peaks shown in $\mathrm{Sr}$ compositions became the AFM peaks in Ba compounds. In Fig. 5.8 (b), the order parameters for the satellite and FM peaks are plotted as a function of $x$, where the drastic split of the two is clearly visible at 0.18 . The magnetic intensities of the satellite peak $\left(\mathrm{I}_{I C}\right)$ and the broadening of the (001) Bragg peak $\left(\mathrm{I}_{F M C}\right)$ represent the degree of the corresponding magnetic coupling, which are compared in panel (c) (Because the crystal size is different for different compositions, the magnetic scattering intensities were normalized by the (001) Bragg peak intensity. To extract only the FM scattering intensity, the data above the transition were subtracted to remove the nuclear contribution. Note that the $\left(\frac{1}{4}, \frac{1}{4}, \frac{1}{4}\right)$ satellite was used for the 0.18 crystal while the $\left(\frac{1}{4}, \frac{1}{4}, \frac{3}{4}\right)$ was used for all other crystals and the normalized intensity was corrected by the square of the magnetic form factor.). Initially, the IC phase is weaker in comparison to the FMC one. However at $x=0.18$, the satellite peaks become resolution limited and the normalized intensity increases sharply. The correlation length of the satellite peak, $\xi_{I C}$, and FM peak, $\xi_{F M}$, are compared in (d). $\xi_{I C}$ and $\xi_{F M}$ are estimated by $\xi=1 / \gamma$, where $\gamma$ is the HWHM of the peaks fitted by Lorentzian function in momentum space. Initially $\xi$ increases slowly with $x$ and is 
comparable in the two phases, leveling off at $x=0.15$ and staying within $1 \mathrm{~nm}$, but by $18 \%$ it rises sharply and becomes quite long for both phases.

Therefore, it can be seen that for $\mathrm{La}_{1-x} \mathrm{Ba}_{x} \mathrm{CoO}_{3}$, as the doping concentration approaches the SG-FM transition boundary, the strengths of both FM and AFM magnetic coupling grew stronger as represented by the scattering intensities, $I$, and the sizes of the two type magnetic clusters grew larger as suggested by $\xi$.

A comparison across the compositions with different dopants, $\mathrm{Ca}^{2+}, \mathrm{Sr}^{2+}$ or $\mathrm{Ba}^{2+}$, gave rise to important information on the magnetic phase separation trends in hole doped cobaltites. Fig. 5.9 showed this trend which is inferred from the correlation length of the FM and the magnetic satellite signals as a function of the average size of the A-site ion, $\left\langle r_{A}\right\rangle$, where $\left\langle r_{A}\right\rangle=(1-x) \cdot r_{L a^{3+}}+x \cdot r_{A^{2+}}$. For the same doping level, $\xi_{F M}$ decreases but $\xi_{I C}$ increases with the increase of $\left\langle r_{A}\right\rangle$. When the dopant changes from $\mathrm{Ca}^{2+}$ to $\mathrm{Sr}^{2+}$ to $\mathrm{Ba}^{2+}$, Ca samples, which correspond to the smallest $\left.<r_{A}\right\rangle$, show the strongest FM signature, but with the second magnetic transition absent. Sr samples, however, have a short-range incommensurate phase, which has a slightly shorter correlation length than the FM phase, but with Ba doping, the short-range incommensurate phase became larger than FM and as doping increased it developed to a long-range and commensurate AFM. The magnetic inhomogeneity, thus, showed a strong dependence on the size of the A-site dopant. 


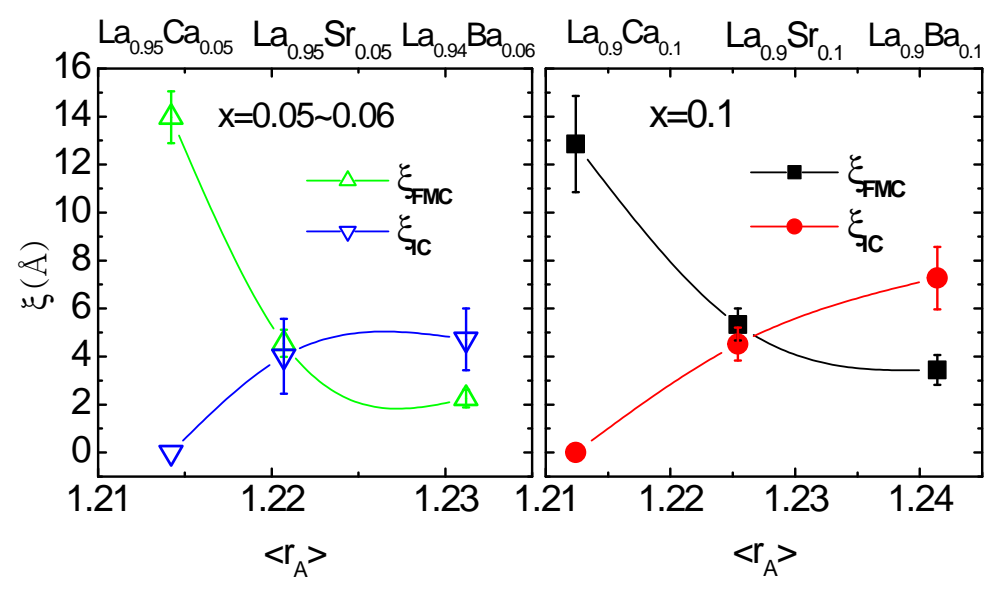

Figure 5.9: The correlation length, $\xi$, as a function of $\left\langle\mathrm{r}_{A}\right\rangle$, determined for the two magnetic components in $\mathrm{Ca}, \mathrm{Sr}$ and Ba crystals of 5(6) and $10 \%$. At a constant hole concentration, $\xi_{F M C}$ is always larger in $\mathrm{Ca}$ than in $\mathrm{Sr}$ and $\mathrm{Ba}$ crystals. No second magnetic phase has been detected in Ca. At the same time, as $\left\langle\mathrm{r}_{A}\right\rangle$ increases, $\xi_{F M C}$ and $\xi_{I C}$ become comparable. The lines are guides to the eye.

\subsection{A Hypothesis about Local Lattice Inhomogene- ity Induced the Magnetic Phase Separation}

In order to understand the occurrence of the magnetic phase separation and its trend with A-site dopants, we noticed that the ionic radius of the A-site dopant, $\mathrm{Ca}^{2+}$, $\mathrm{Sr}^{2+}$ and $\mathrm{Ba}^{2+}$, is quite different than the one of the original $\mathrm{La}^{3+}$ (the difference is at the order of $\left.10^{-1} \AA[77]\right)$. Therefore, considerable steric effects should be expected. The difference on the ionic size of A-site dopant and the original ion can have an effect on both average lattice and the local lattice, especially on the local lattice that is close to the dopant if the size difference is significant.

The magnetic phase separation and its trend may be attributed to the size effects 


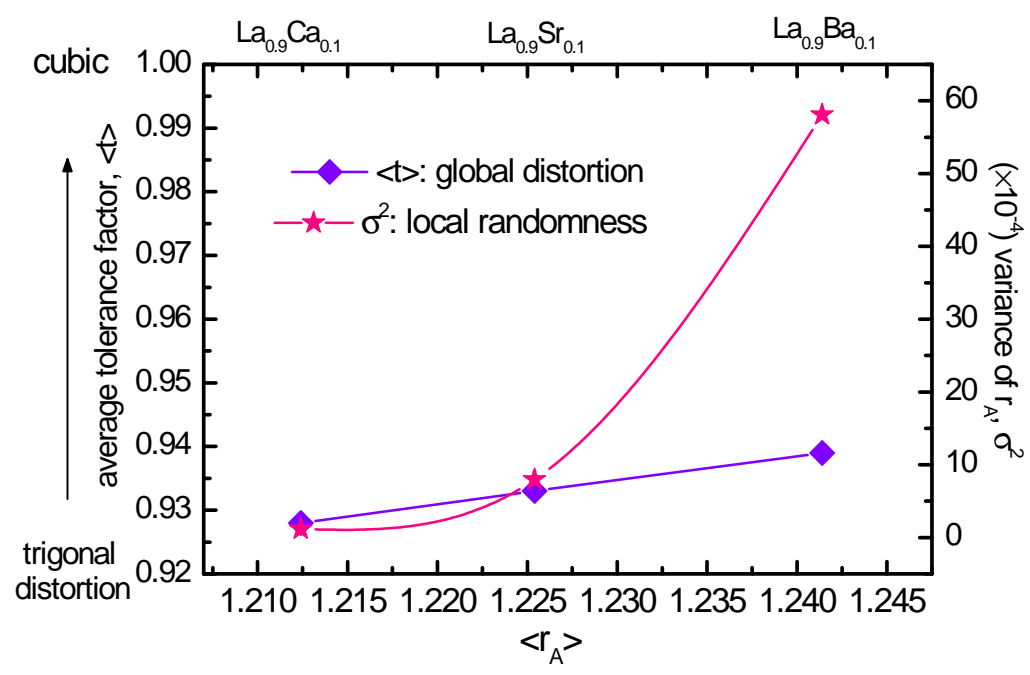

Figure 5.10: The average tolerance factor, $\left\langle t>\right.$, and its variance, $\sigma^{2}$, as a function of the average A-site ionic size, $\left\langle r_{A}\right\rangle$, in 10\% doped $\mathrm{Ca}$, Sr and Ba compositions.

on both the average and local lattice, or may be due to one of them. To find out which effect could be the direct cause, first of all it is useful to understand what the size effects do to the average and the local lattice. Two parameters are chosen to describe this situation: the average tolerance factor, $\langle t\rangle\left(\langle t\rangle=\frac{\left\langle r_{A}\right\rangle+r_{O}}{\sqrt{2}\left(<r_{B}>+r_{O}\right)}\right)$, is used to represent the average effect on the overall lattice with doping; the variance of $\mathrm{r}_{A}, \sigma^{2}\left(\sigma^{2}=(1-x) \cdot r_{L a}^{2}+x \cdot r_{A}^{2}-<r_{A}>^{2}\right)$, is the differences of the ionic size of A-site ion around their mean value so it can be used to describe the disturbance on the local lattice caused by the dopants; in other words, the local randomness caused by the local lattice inhomogeneity.

Fig. 5.10 plots the dependence of $\left\langle t>\right.$ and $\sigma^{2}$ with $\left\langle r_{A}>\right.$ for $10 \%$ doped samples. The plotted values of $\langle t\rangle$ are calculated from the empirical ionic radii 
listed in ref [77]. The values of $\langle t\rangle$ can also be estimated from the results of Rietveld refinement which is listed in the Table 5.1. It can be seen that as the dopant changes from $\mathrm{Ca}^{2+}$ to $\mathrm{Sr}^{2+}$ to $\mathrm{Ba}^{2+},<t>$ increases almost linearly towards the value 1 , which indicates the average lattice approaches the ideal cubic symmetry and releases the trigonal distortion. This means that the bent M-O-M bond angle in the $\mathrm{R} \overline{3} \mathrm{c}$ symmetry is straightened to be close to $180^{\circ}$ as the dopant changed from $\mathrm{Ca}^{2+}$ to $\mathrm{Sr}^{2+}$ to $\mathrm{Ba}^{2+}$. The straightened M-O-M bond angle increases the strength of FM coupling $[16,20]$, so this average effect may mispredict that the FM feature became stronger from $\mathrm{Ca}^{2+}$ to $\mathrm{Sr}^{2+}$ to $\mathrm{Ba}^{2+}$. This is clearly opposite with what was observed. On the other hand, it can be seen from Fig. 5.10 that $\sigma^{2}$ rises at a much faster rate which indicates a much rapid increase of the local lattice disturbance. The increased tendency to form AFM phase as the dopant changes from $\mathrm{Ca}^{2+}$ to $\mathrm{Sr}^{2+}$ to $\mathrm{Ba}^{2+}$, thus, is correlated with the increased local lattice disturbance.

To further understand the disturbance on the local lattice, the local tolerance factor, $t$, is used to describe a specific region where a certain group of $\mathrm{A}$ and $\mathrm{B}$ ions are involved, because the change in the $A-O$ and $B-O$ bond lengths with doping ultimately depends on the kinds of neighbors. For example, in the dopant-rich region, the $\mathrm{Co}^{4+}$ ions are most likely located in the immediate vicinity of the divalent dopant $\mathrm{A}^{2+}$, giving rise to the corresponding $t$, which is referred to as $t_{\text {dopant-rich }}$, at the value of $\sim 1.00$ for Ba doping (To evaluate the value of $t$, the nominal ionic size for $\mathrm{Co}^{4+}$ in the HS configuration in a VI coordinated environment was used because of its 
availability [77], but it can be expected that for other possible spin states of the $\mathrm{Co}^{4+}$ ion, the ionic size would be smaller, implying that the value of $t$ can be even slightly greater than 1). For the region that is far away from the dopant, referred to as the background region, the lattice is most close to the pure compound, $\mathrm{LaCoO}_{3}$, for which $t_{\mathrm{LaCoO}_{3}}=0.957<1$ in its ground state with $\mathrm{Co}^{3+}$ at the LS state. The corresponding $t$ for the background region is thus $t_{\text {background }} \sim t_{\mathrm{LaCoO}_{3}}=0.957$. Noticing that $t_{\text {dopant-rich }} \sim 1.00$ and $t_{\text {background }} \sim 0.957$, the lattice must experience some kind of adjustment, because the $t$ values for this two regions are considerably different. This adjustment on the lattice can be realized in the regions that are located in between the dopant-rich area and background.

Table 5.1 lists the values of $t_{\text {dopant-rich }}$ for the three systems along with the percent change from the parent compound. The value of $\langle t\rangle$ is also listed, which is evaluated from the crystallographic refinement results. The data for the Sr doped was obtained from Ref. [74]. This method only provides an average effect of the lattice from the substitution and there is no reason to assume that the lattice in the various region would behave in exactly the same way as quantified by $\langle t\rangle$, especially when the steric effects induced by the dopant are significant.

It can be seen that in the case where the trend is to positively increase the tolerance factor and the percent change is large compared with the parent compound, the AFM correlations tend to be observed concomitantly with the FM ones. But in the Ca doped compounds the percent change is small and negative, and in this case the 


\begin{tabular}{lcccc}
\hline \hline & $<t>$ & $\mathbf{t}_{\text {dopant-rich }}$ & $\%$ enhancement & AFM feature \\
\hline \hline $\mathrm{Ba}^{2+}, \mathrm{Co}^{4+}, \mathrm{O}^{2-}$ & 0.9653 & 1.061 & $10.867 \%$ & short-ICM to long-CM \\
$\mathrm{Sr}^{2+}, \mathrm{Co}^{4+}, \mathrm{O}^{2-}$ & 0.9662 & 1.000 & $4.493 \%$ & short-range ICM \\
$\mathrm{Ca}^{2+}, \mathrm{Co}^{4+}, \mathrm{O}^{2-}$ & 0.9582 & 0.952 & $-0.522 \%$ & no AFM \\
\hline \hline
\end{tabular}

Table 5.1: A list of tolerance factors, $t$, evaluated only near the dopant rich regions for $\mathrm{Ca}, \mathrm{Sr}$ and $\mathrm{Ba}$ doping. The $<\mathrm{t}>$ are calculated from values obtained from the crystallographic refinement at room temperature for the samples with doping $\mathrm{x}=\mathrm{y}=0.1$. t-dopint-rich is evaluated considering only the immediate vicinity of the dopant; the percent increase is evaluated based on the comparison with the t of $\mathrm{LaCoO}_{3}$. ICM refers to incommensurate ordering while $\mathrm{CM}$ refers to commensurate magnetic ordering.

AFM correlations are absent.

Therefore, the large difference in local $t$ between the dopant-rich region and the background region appears to favor the formation of the AFM phase. The change of local $t$ suggests an inhomogeneous lattice. A corona model is therefore put forward to describe a possible mechanism that the magnetic phase separation is driven by the inhomogeneity of the lattice.

In this corona model (shown in Fig. 5.11) the dopant distribution, lattice, magnetic correlations and electrical conduction are all coupled. There are three representative regions (labeled as (1), (2) and (3)) where the slight difference in the local lattice determines whether the spins are FM or AFM coupled. Region (1) is the dopant-rich-FM region (shown as a red zone): as it is discussed above, for $\mathrm{Sr}$ and $\mathrm{Ba}$ doping this region has the largest $t$ value which is $\sim 1$, indicating the local lattice is the most close to cubic. The resulting M-O bonds are more straightened and thus more likely to establish FM coupling via the DE through $\mathrm{Co}^{4+}-\mathrm{O}_{-} \mathrm{Co}^{3+}$ since the gen- 


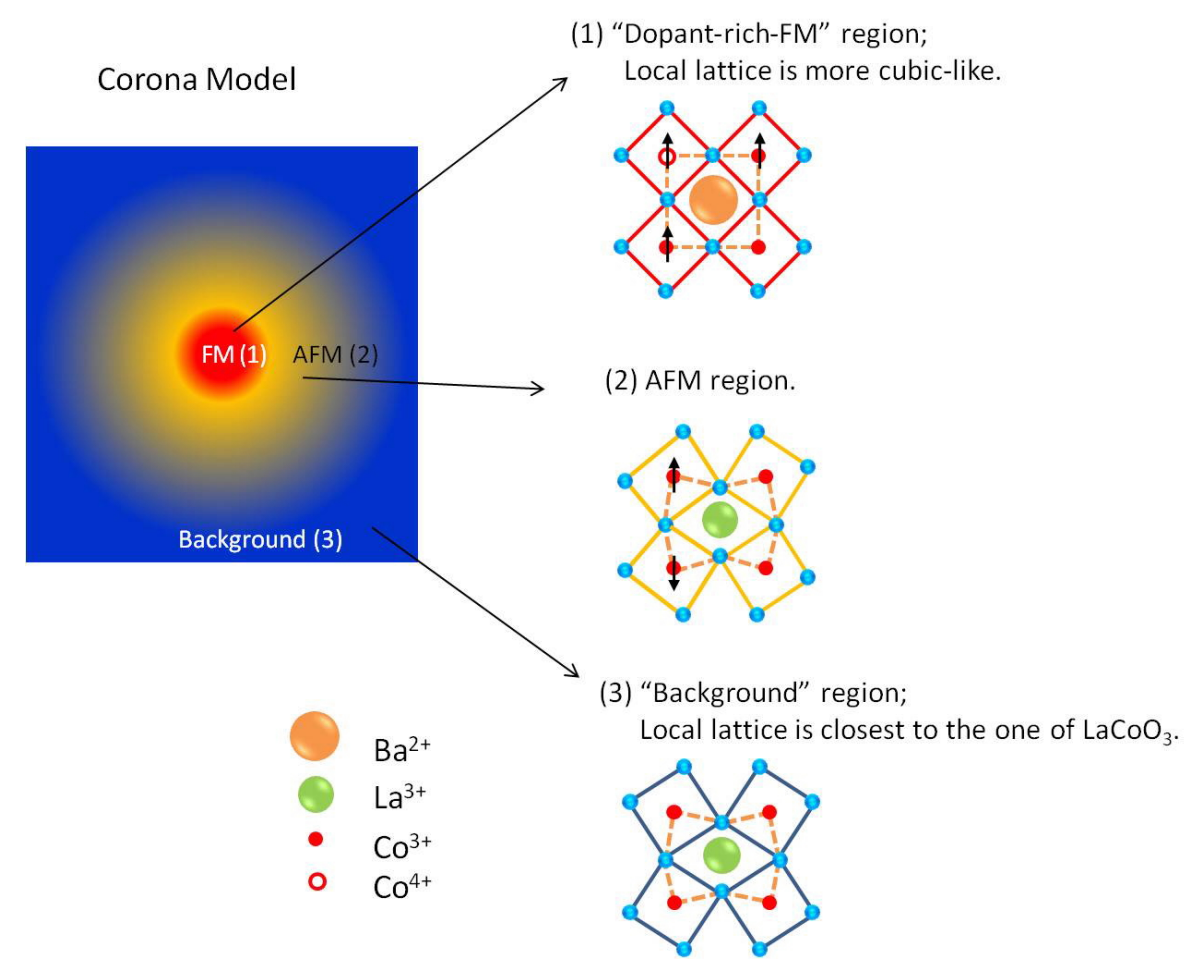

Figure 5.11: The corona model discribes three different local lattices in $\mathrm{La}_{1-x}(\mathrm{Sr} / \mathrm{Ba})_{x} \mathrm{CoO}_{3}:$ (1) "Dopant-rich-FM" region shown as a red zone. In this area, the large size of the dopant leads the lattice to be more cubic-like, and the FM coupling is established via the DE through $\mathrm{Co}^{4+}-\mathrm{O}_{-} \mathrm{Co}^{3+}$. (2) AFM region, shown as the yellow corona, is the area connecting the region (1) and the background (3). For the background (blue), its lattice properties is most close to the pure compound, $\mathrm{LaCoO}_{3}$, maintaining a significant trigonal distortion. Thus, the local lattice of region (2) gradually transfers the difference on the local lattice between (1) and (3). The AFM coupling is established between two Co ions with identical spin configuration via super-exchange. The right panel gave a schematic of two-dimension lattices in each region, to demonstrate the possible differences on the local lattices. 
erated $\mathrm{Co}^{4+}$ ions due to hole doping are located around the dopants. Far away from the "dopant-rich-FM zone", region (3) represents the background matrix (blue color), where the lattice is least disturbed by doping, maintaining a rhombohedral distortion $(\mathrm{R} \overline{3} \mathrm{c})$ with $t \sim 0.957$. For the region (2) which connects $(1)$ and (3), shown as the yellow corona, the local lattice is unevenly distorted in a manner that the transition of the lattice from a cubic-like to a rhombohedrally distorted one (i.e. from $t \sim 1$ to 0.957 ) is smooth and gradual. It is this irregularly distorted lattice that accommodates the AFM correlations. The AFM coupling most likely happens between two $\mathrm{Co}^{3+}$ ions on the same spin state, i.e., $\mathrm{Co}^{3+}(\mathrm{IS})-\mathrm{O}-\mathrm{Co}^{3+}(\mathrm{IS}), \mathrm{Co}^{3+}(\mathrm{HS})-\mathrm{O}-\mathrm{Co}^{3+}(\mathrm{HS})$. On the other hand, because the size of the Co ion varies depending on its valence and spin states, the possible existence of Co ions at various valency and spin states provides the room for the value of $t$ to have a slight change, i.e. an irregular distortion on the lattice.

For Ca doping, however, because the $t$ values of region (1) and (3) are so close (Table 4.1), meaning the difference between the lattice of dopant-rich-FM region and the background is small, the transition area, region (2), along with the AFM phase is absent. On the other hand, For Ba doping, the difference between the local lattice (1) and (3) is the largest (Table 4.1), so that the degree and extent of irregular lattice distortion in the region (2) is the largest, which results in the strongest AFM feature. For the Sr doping, the situation lies in between Ca and Ba doping. The competition between the FM and AFM/IC phase determines the FM-IMT. From Ca to Sr to Ba, 
the AFM feature becomes stronger and therefore caused such a dopant dependent FM-IMT.

In short, the dopant distribution, lattice, magnetic correlations and the electrical conductivity are all coupled. From experiments, it was reported based on the electron microscopy of $15 \%$ and $30 \%$ doped $\mathrm{Sr}$ compositions that there are $\mathrm{Sr}^{2+}$ rich and poor regions [14].

The crystal symmetry, however, may be best described by a lower symmetry if the degree of inhomogeneity is significant. In the neutron elastic scattering measurements on single crystal a peak at $\left(\frac{1}{2}, \frac{1}{2}, \frac{1}{2}\right)$ position, i.e. $Q \sim 1.43 \AA^{-1}$ (Fig.5.7(a)), was observed in both Sr- and Ba- compounds beginning from the low temperatures. This $Q$ value is shorter than the smallest values of the Bragg peaks allowed by $\mathrm{R} \overline{3} \mathrm{c}$ symmetry, which is $\sim 1.65 \AA^{-1}$, but it is allowed by the lower symmetry such as $\mathrm{R} \overline{3}$ or monoclinic. As a comparison, by using the same experiment setup this peak was not shown at all in the crystal of $\mathrm{LaCo}_{1-y} \mathrm{Ni}_{y} \mathrm{O}_{3}$ (Fig. 6.6) for which the average lattice symmetry is $R \overline{3} \mathrm{c}$ and at the same time no local lattice distortion was observed based on PDF analysis (details in Chap 6.2 and 6.3). The crystal structure studies based on the X-ray diffractions further suggested the monoclinic symmetry with space group I2/ $a$ in $\mathrm{R}_{1-x} \mathrm{Sr}_{x} \mathrm{CoO}_{3}(\mathrm{R}=\mathrm{La}, \mathrm{Pr}$ and $\mathrm{Nd})$ [83][52] where the break of $\mathrm{R} \overline{\mathrm{c}}$ symmetry was argued to be caused by the local lattice distortion. 


\subsection{Summary}

The magnetic phase separation trends in hole doped cobaltites, $\mathrm{La}_{1-x} \mathrm{~A}_{x} \mathrm{CoO}_{3}$ $\left(\mathrm{A}=\mathrm{Ca}^{2+}, \mathrm{Sr}^{2+}\right.$, and $\left.\mathrm{Ba}^{2+}\right)$, are characterized by using elastic neutron scattering on single crystals. It was found that below the global magnetic transition, the development of magnetic superstructures is strongly dependent on the size of the A-site dopant, $r_{A}$. Upon reducing the $r_{A}$ i.e., as with Ca doping, only a commensurate ferromagnetic cluster phase is evident. On expanding the $r_{A}$, the tendency of coexistence of competing ferromagnetic and antiferromagnetic orders increases giving rise to an inhomogeneous magnetic ground state. The antiferromagnetic ordered state, initially incommensurate, continuously strengthens and becomes commensurate with long-range order and a characteristic cubic wave vector of $Q_{c}=(0.25,0.25,0.25)$. As the hole doping concentration increases to approach the SG-FM transition boundary, both order parameters become stronger and comparable in magnitude indicative of the phase-separated nature of the cobalt perovskite system. Considering that the large ionic size variance can cause an inhomogenous local lattice, a corona model was proposed as a possible mechanism by which the magnetic phase separation is driven by the inhomogeneous local lattice, where the AFM regions are created by the large difference in the local lattices between the dopant-rich FM region and the background region. 


\section{Chapter 6}

\section{Unconventional Magnetism in the}

\section{Insulating State of $\mathrm{LaCo}_{1-y} \mathrm{Ni}_{y} \mathrm{O}_{3}$}

\subsection{Effects of Doping Magnetic Ion $\mathrm{Ni}^{3+}$ and $\mathrm{Fe}^{3+}$ in $\mathrm{LaCoO}_{3}$}

In the last chapter, it was shown that the addition of holes instigates the excitation of the $\mathrm{Co}^{3+}$ ion to magnetic states and a global FM-metallic state is realized regardless of whether the dopant is $\mathrm{Ca}^{2+}, \mathrm{Sr}^{2+}$ or $\mathrm{Ba}^{2+}$. While hole doping actually introduces the magnetic ion $\mathrm{Co}^{4+}$ onto the B-site: $\mathrm{La}_{1-x}^{3+} \mathrm{A}_{x}^{2+} \mathrm{Co}_{y}^{4+} \mathrm{Co}_{1-y}^{3+} \mathrm{O}_{3}^{2-}(x=y)$, one may wonder whether a global state can be also achieved by direct replacing $\mathrm{Co}^{3+}$ with other magnetic ions? To this end, $\mathrm{LaCo}_{1-y} \mathrm{Ni}_{y} \mathrm{O}_{3}$ and $\mathrm{LaCo}_{1-y} \mathrm{Fe}_{y} \mathrm{O}_{3}$ were chosen. The Bsite dopant ion $\mathrm{Fe}^{3+}\left(3 d^{5}\right)$ has one fewer electron than the original $\mathrm{Co}^{3+}$ ion $\left(3 d^{6}\right)$, 
while $\mathrm{Ni}^{3+}\left(3 d^{7}\right)$ has one more electron than $\mathrm{Co}^{3+}$. The fact that the substitution takes place at the B-site with the valence state of $3+$ and not at the A-site with a valence of $2+$ is confirmed by XANES measurements which determined the valence of $\mathrm{Ni}$ and Co ions both to be trivalent [45].

The doped compounds $\mathrm{LaCo}_{1-y} \mathrm{Ni}_{y} \mathrm{O}_{3}$ and $\mathrm{LaCo}_{1-y} \mathrm{Fe}_{y} \mathrm{O}_{3}$ have essentially different bulk magnetic and electric transport properties. Pure $\mathrm{LaNiO}_{3}$ is a good metal with an enhanced Pauli paramagnetic susceptibility, suggesting that the $e_{g}$ electron of the $\mathrm{Ni}^{3+}$ ion is highly mobile: $t_{2 g}^{* 6} \sigma^{* 1}[25,54,26]$. The mixed compound of $\mathrm{LaNiO}_{3}$ and $\mathrm{LaCoO}_{3}$, i.e. $\mathrm{LaCo}_{1-y} \mathrm{Ni}_{y} \mathrm{O}_{3}$, evolves from a spin-glass state to a $\mathrm{FM}$ state as $y$ exceeds $\sim 10 \%$, and the IMT occurred at a much higher doping concentration, $y$ $\sim 40 \%$ [29]. On the other hand, pure $\mathrm{LaFeO}_{3}$ is an AFM insulator which indicates that the localized character of the $e_{g}$ electrons of $\mathrm{Fe}^{3+}$ ion: $t_{2 g}^{* 3} e_{g}^{* 2}[25,26]$. The mixed compound of $\mathrm{LaFeO}_{3}$ and $\mathrm{LaCoO}_{3}$, i.e. $\mathrm{LaCo}_{1-y} \mathrm{Fe}_{y} \mathrm{O}_{3}$, is an insulator and primarily remains in the paramagnetic state. When the Fe concentration is as high as $\sim 40 \%$, a weak AFM order appears, and its magnetic structure was found to be G-type which is similar to $\mathrm{LaFeO}_{3}[38]$ based on the neutron powder diffraction refinement.

The bulk magnetic susceptibility measurements demonstrate the striking difference between the doping effects of $\mathrm{Ni}$ and $\mathrm{Fe}$. The magnetization measurements were made in $d c$ mode under the applied field of 200 Oe using the ACMS option of a Quantum Design Physical Property Measurement System. The powder compounds, $\mathrm{LaCo}_{0.9} \mathrm{Ni}_{0.1} \mathrm{O}_{3}$ and $\mathrm{LaCo}_{0.6} \mathrm{Ni}_{0.4} \mathrm{O}_{3}$ (the data in black) and $\mathrm{LaCo}_{0.9} \mathrm{Fe}_{0.1} \mathrm{O}_{3}$ 


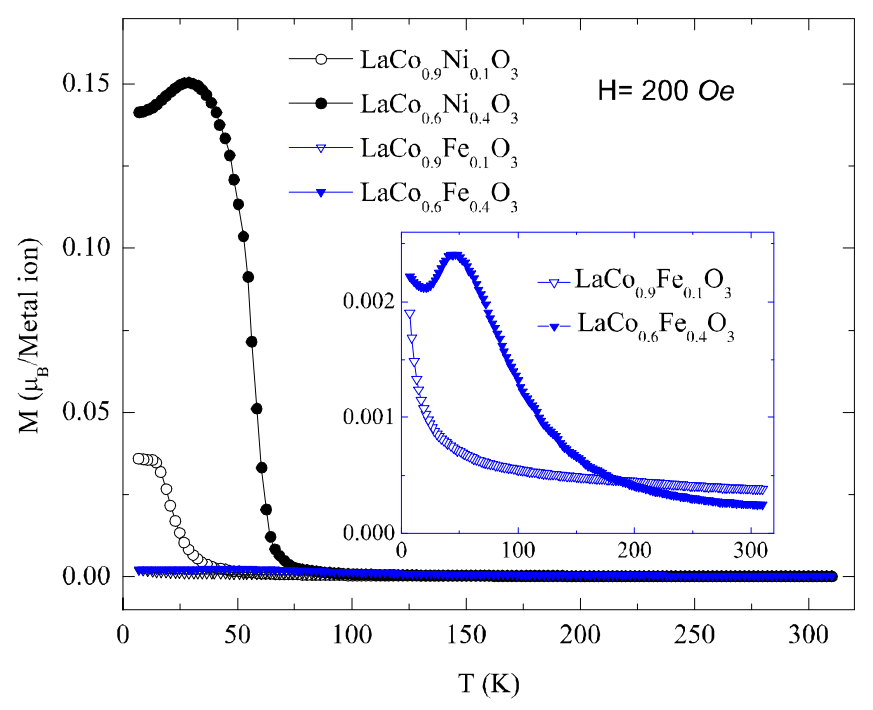

Figure 6.1: Bulk magnetization as a function of temperature with dc mode under 200 Oe field in $\mathrm{LaCo}_{1-y} \mathrm{Ni}_{y} \mathrm{O}_{3}$ and $\mathrm{LaCo}_{1-y} \mathrm{Fe}_{y} \mathrm{O}_{3}$.

and $\mathrm{LaCo}_{0.6} \mathrm{Fe}_{0.4} \mathrm{O}_{3}$ (the data in blue ) are measured. Fig. 6.1 shows the temperature dependence of the field-cooled magnetization for $\mathrm{LaCo}_{1-y} \mathrm{Ni}_{y} \mathrm{O}_{3}$ and $\mathrm{LaCo}_{1-y} \mathrm{Fe}_{y} \mathrm{O}_{3}$. The inset is an enlarged view of Fe- samples. Clearly Ni doped compounds have much larger magnetization than Fe doped ones. For $\mathrm{LaCo}_{1-y} \mathrm{Ni}_{y} \mathrm{O}_{3}$, the significant increase of the magnetization in $\mathrm{LaCo}_{0.6} \mathrm{Ni}_{0.4} \mathrm{O}_{3}$ results from the FM state, while for $\mathrm{LaCo}_{1-y} \mathrm{Fe}_{y} \mathrm{O}_{3}$ the magnetization still follows the Curie-Weiss paramagnetic curve in $\mathrm{LaCo}_{0.9} \mathrm{Fe}_{0.1} \mathrm{O}_{3}$. These results are consistent with the reports of other groups $[41,3,29,87,38]$. Therefore, it appears that Ni doping significantly promotes the magnetization but Fe doping does not. 


\subsection{Spin States of $\mathrm{Co}^{3+}$ Ion in Trigonal Lattice}

In order to understand the essential difference of $\mathrm{Ni}$ and Fe doping on the compounds' physical properties, apparently only considering the role of dopant $\mathrm{Ni}$ and Fe is not enough. For example, for Ni doped compounds, one might naively think that the introduction of $\mathrm{Ni}$ onto the Co site in $\mathrm{LaCoO}_{3}$ would not cause magnetic ordering since $\mathrm{LaNiO}_{3}$ is a Pauli paramagnet. The ground state of $\mathrm{Co}^{3+}$ ions after doping, therefore, should play a critical role.

There are some early studies of this issue but most of them are based on the bulk properties. In our efforts, we investigated the states of $\mathrm{Co}^{3+}$ with $\mathrm{Ni} / \mathrm{Fe}$ doping on an atomic scale by neutron scattering. The atomic properties of $\mathrm{LaCo}_{1-y} \mathrm{Ni}_{y} \mathrm{O}_{3}$ and $\mathrm{LaCo}_{1-y} \mathrm{Fe}_{y} \mathrm{O}_{3}$ with $y=0.1$ and 0.4 were examined via both Rietveld and PDF methods [96].

For the powder samples, the neutron-diffraction measurements were carried out from 12 to $300 \mathrm{~K}$ using time-of-flight machine NPDF at Los Alamos National Laboratory. The samples were sealed in a vanadium can with helium as the exchange gas and loaded in a displex refrigeration system. The Rietveld refinement of the compositions of $\mathrm{LaCo}_{1-y} \mathrm{Ni}_{y} \mathrm{O}_{3}$ and $\mathrm{LaCo}_{1-y} \mathrm{Fe}_{y} \mathrm{O}_{3}(y=0.1$ and 0.4$)$ showed that the lattice symmetries maintained a trigonal distortion with the space group $\mathrm{R} \overline{\mathrm{c}} \mathrm{c}$ in both doped systems. Table 6.1 lists the results of Rietveld refinement of the crystal structure as a function of temperature. As it can be seen, the symmetry remains rhombohedral

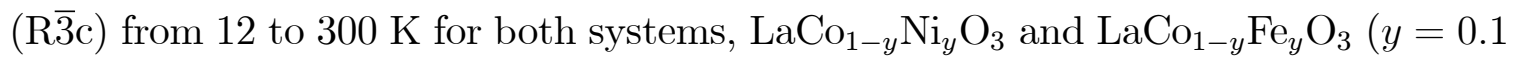




\begin{tabular}{|c|c|c|c|c|}
\hline$\overline{\mathrm{LaCo}_{1-y} \mathrm{Ni}_{y} \mathrm{O}_{3}}$ & & 0.1 & & \\
\hline $\mathrm{T}(\mathrm{K})$ & $a(\AA)$ & $\alpha^{\circ}$ & $x$ & $\mathrm{R}_{w p}(\%)$ \\
\hline 12 & $5.34574(4)$ & $61.006(1)$ & $0.19766(3)$ & 3.94 \\
\hline 50 & $5.34791(3)$ & $60.998(1)$ & $0.19778(3)$ & 3.86 \\
\hline 100 & $5.35240(3)$ & $60.971(1)$ & $0.19807(3)$ & 3.81 \\
\hline 295 & $5.37236(2)$ & $60.838(1)$ & $0.19971(4)$ & 3.59 \\
\hline $\mathrm{LaCo}_{1-y} \mathrm{Fe}_{y} \mathrm{O}_{3}$ & & 0.1 & & \\
\hline 12 & $5.3638(1)$ & $60.915(1)$ & $0.19734(8)$ & 4.33 \\
\hline 50 & $5.3644(1)$ & $60.911(1)$ & $0.19737(8)$ & 4.27 \\
\hline 100 & $5.3714(1)$ & $60.891(1)$ & 0.1976 & 3.70 \\
\hline 295 & $5.3850(1)$ & $60.777(1)$ & $0.19926(9)$ & 3.96 \\
\hline 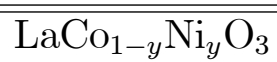 & & $\overline{0.4}$ & & \\
\hline $\mathrm{T}(\mathrm{K})$ & $a(\AA)$ & $\alpha^{\circ}$ & $x$ & $\mathrm{R}_{w p}(\%)$ \\
\hline 12 & $5.36044(4)$ & $61.033(1)$ & $0.19750(5)$ & 4.29 \\
\hline 50 & $5.36248(4)$ & $61.027(1)$ & $0.19758(5)$ & 4.43 \\
\hline 100 & $5.36487(5)$ & $61.002(1)$ & $0.19788(4)$ & 4.40 \\
\hline 295 & $5.38414(5)$ & $60.871(1)$ & $0.19954(5)$ & 4.03 \\
\hline $\mathrm{LaCO}_{1-y} \mathrm{Fe}_{y} \mathrm{O}$ & & 0.4 & & \\
\hline 12 & $5.39352(8)$ & $60.863(1)$ & 0.1961 & 5.15 \\
\hline 50 & $5.39402(9)$ & $60.859(1)$ & $0.19613(6)$ & 5.11 \\
\hline 100 & $5.39828(7)$ & $60.845(1)$ & $0.19634(4)$ & 4.23 \\
\hline 295 & $5.41035(6)$ & $60.753(1)$ & $0.19767(4)$ & 3.43 \\
\hline
\end{tabular}

Table 6.1: The Rietveld refinement results on $\mathrm{LaCo}_{1-y} \mathrm{~B}_{y} \mathrm{O}_{3}$ for $\mathrm{B}=\mathrm{Ni}$ and $\mathrm{Fe}, \mathrm{y}=$ 0.1 and 0.4 from $\mathrm{T}=12-295 \mathrm{~K}$. The listed parameters refer to space group R-3c (No. 167) in the rhombohedral unit cell. The La position (2a) is at $(1 / 4,1 / 4,1 / 4)$, the $\mathrm{Co} / \mathrm{Ni} / \mathrm{Fe}(2 \mathrm{~b})$ is at $(0,0,0)$ and the oxygen position $(6 \mathrm{e})$ is given by $(-\mathrm{x}, 1 / 2+\mathrm{x}$, $3 / 4)$. Listed is the lattice constants and weighted R-factor.

and 0.4 , which is consistent with other reports in the literature $[45,60,87]$. As the temperature rises, the lattice shows a slight expansion while the rhombohedral angle, $\alpha$, gets closer to $60^{\circ}$ in all samples. From $y=0.1$ to 0.4 , the lattice shows a less than $0.5 \%$ expansion in both systems.

At a first glance, it appears that doping $\mathrm{Ni}$ and Fe have the same effects, i.e., for both dopings, the lattice symmetry remains the same as that for the parent compound 
$\mathrm{LaCoO}_{3}$, but a close examination showed that the effects of trigonal distortions on the $\mathrm{BO}_{6}$ octahedron are different in two systems.

Compared with the ideal cubic structure with the space group $\operatorname{Pm} \overline{3} \mathrm{~m}$, the $\mathrm{R} \overline{3} \mathrm{c}$ symmetry is realized by a rotation of the nearly-regular $\mathrm{BO}_{6}$ octahedra around the trigonal axis, which is the body diagonal [111] of the rhombohedral cell. After lowering the symmetry from cubic to trigonal, the [111] becomes an unique direction in the crystal. Here 'regular' refers to the highly symmetric shape of an octahedron $\left(O_{h}\right)$ in cubic and 'nearly-regular' refers to the possible deformation on an octahedron that is allowed by the trigonal point group. Following the notation of Ref. [55], the degree of octahedral rotation, $\omega$, is determined from the oxygen coordinates, $x$ :

$$
\omega=\tan ^{-1} 4 \sqrt{3}\left(\frac{x}{2}+\frac{1}{8}\right)
$$

In the ideal cubic symmetry, $x=-0.25$ (thus, $\omega=0$ ) and the rhombohedral angle $\alpha=60^{\circ}$. However, $\alpha$ is independent of $\omega$ or $x$. This means when the crystal structure is lowered from $\operatorname{Pm} \overline{3} \mathrm{~m}$ to $\mathrm{R} \overline{3} \mathrm{c}$, two parameters, $x$ and $\alpha$, are allowed to change away from their original values, and there is no direct correlation between the two.

Fig. $6.2(\mathrm{~b})$ and (d) show the refinement results on $\omega$ and $\alpha$ as a function of doping in $\mathrm{LaCo}_{1-y} \mathrm{Ni}_{y} \mathrm{O}_{3}$ and $\mathrm{LaCo}_{1-y} \mathrm{Fe}_{y} \mathrm{O}_{3}$ at RT; (a) and (c) are the data of $\omega$ and $\alpha$ in the hole doped system $\mathrm{La}_{1-x} \mathrm{Ba}_{x} \mathrm{CoO}_{3}$. From (a) and (c), we can see that as the Ba doping increases, $\omega$ decreases and approaches $0^{\circ}$ while $\alpha$ approaches $60^{\circ}$. These two effects combined indicate that the overall symmetry approaches cubic. However, in $\mathrm{Ni}$ or $\mathrm{Fe}$ doped compounds we first noticed that the change in the values of $\omega$ 


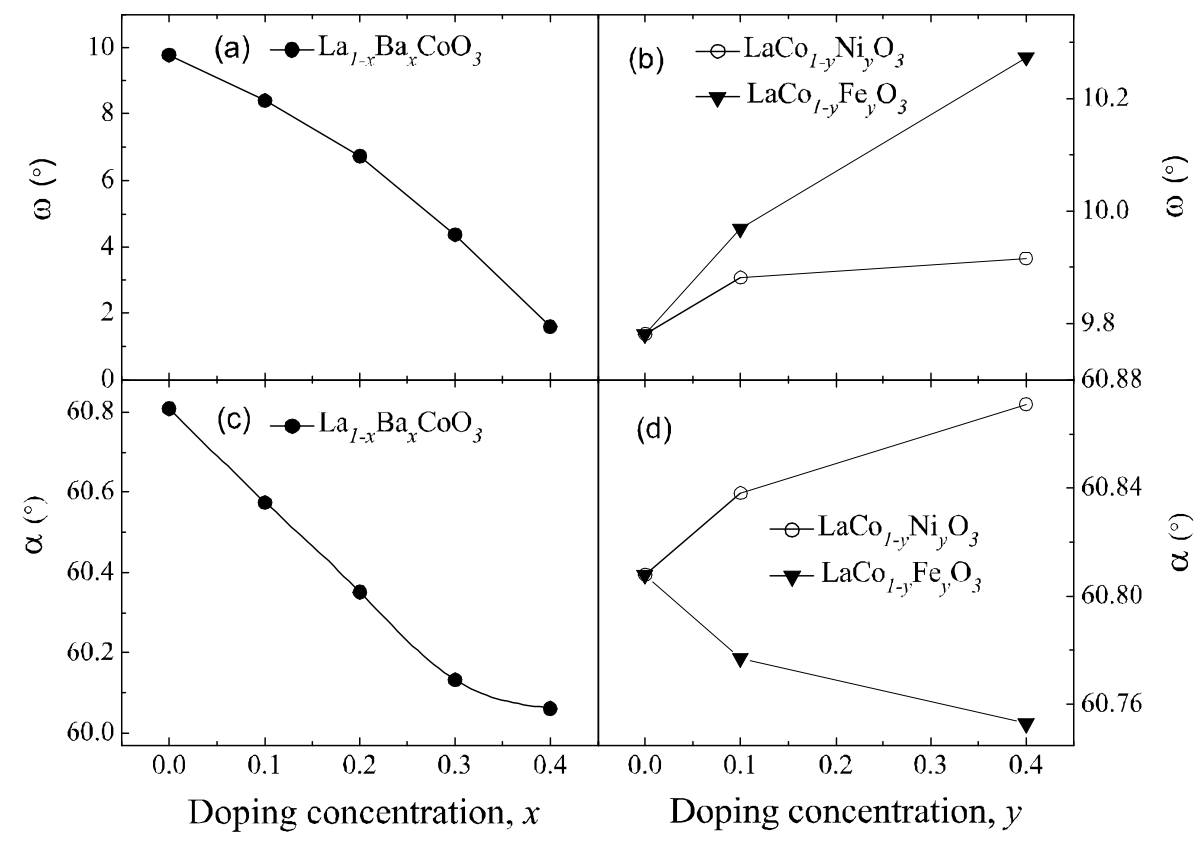

Figure 6.2: The octahedron rotation angle, $\omega$, and the rhombohedral unit cell angle, $\alpha$, as a function of doping concentrations at $\mathrm{RT}$ for $\mathrm{La}_{1-x} \mathrm{Ba}_{x} \mathrm{CoO}_{3}$ (panel (a) and (c)) and $\mathrm{LaCo}_{1-y} \mathrm{Ni}_{y} \mathrm{O}_{3}$ and $\mathrm{LaCo}_{1-y} \mathrm{Fe}_{y} \mathrm{O}_{3}$ (panel (b) and (d)). 
and $\alpha$ are not as large as those in $\mathrm{La}_{1-x} \mathrm{Ba}_{x} \mathrm{CoO}_{3}$. This is not surprising considering that the difference in the nominal ionic sizes of the B-site ions ( $\mathrm{Ni} / \mathrm{Fe}$ versus $\mathrm{Co}$ ) is almost negligible if compared with the difference of the A-site ions in $\mathrm{La}_{1-x} \mathrm{Ba}_{x} \mathrm{CoO}_{3}$. For example: $r_{N i_{L S}^{3+}}-r_{C o_{L S}^{3+}}=0.015$ and $r_{N i_{L S}^{3+}}-r_{C o_{H S}^{3+}}=-0.05$, compared with $r_{\mathrm{Ba}^{2+}}-r_{\mathrm{La}^{3+}}=0.254$ in a IX coordinated environment [77]. At the same time, it can be clearly seen that doping with Ni has a different trend than with Fe: panel (b) shows that $\omega$ increases with doping for both compounds, but in (d) $\alpha$ increases away from the ideal $60^{\circ}$ for $\mathrm{Ni}$ doped samples, but decreases towards $60^{\circ}$ for Fe doped ones. This difference, therefore, is difficult to be simply understood as the result of "size effect'.

The spin state of $\mathrm{Co}^{3+}$ ion is sensitive to its environment, so what do these two uncorrelated parameters which describe the trigonal distortion mean in terms of the oxygen octahedron? First, $\omega$ is the rotation angle of the octahedron around [111] axis. In the cubic symmetry, oxygen ions sit midway between the cubic sides, but as the symmetry is lowered to rhombohedral, the oxygen ions move away from these high symmetry positions. In the Fig. $6.3, \omega$ is indicated as $\angle D O A$, where $\mathrm{D}$ is the midpoint of the cubic side, $\mathrm{O}$ is the intersection point of [111] axis with the octahedron, and $\mathrm{A}$ is the actual position of oxygen in the rhombohedral symmetry.

The rhombohedral unit cell angle, $\alpha$, however, is related to the distortion of the octahedron in a more complicated way. $\alpha$ is a typical parameter but not able to provide a direct description of $\mathrm{BO}_{6 / 2}$ octahedron itself. The shape of the $\mathrm{BO}_{6 / 2}$ 


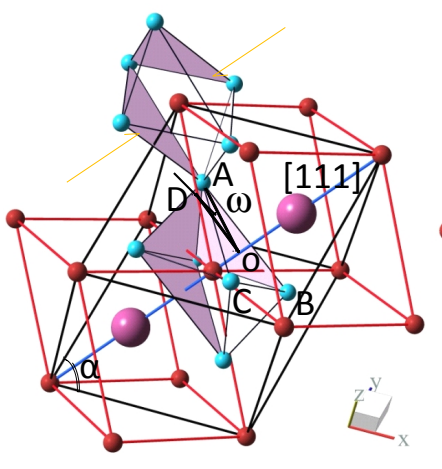

(i) Deformed octahedron

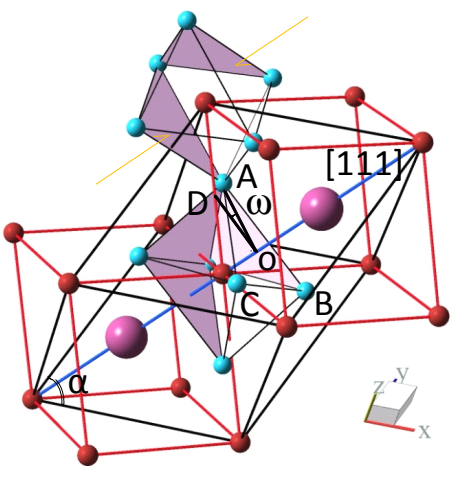

(ii) Release of deformation

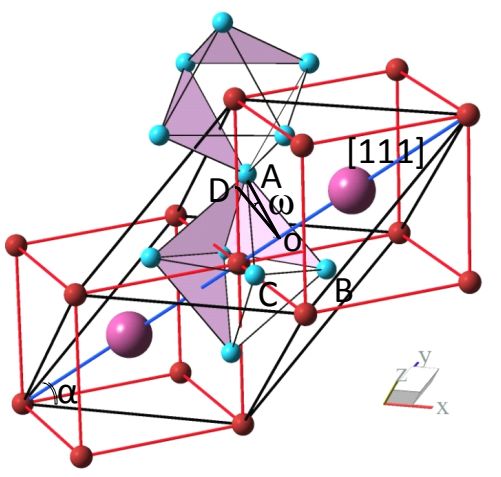

(iii) Undeformed octahedron

Figure 6.3: A schematic of the deformation. The black lines define one rhombohedral unit cell and the red lines indicate two constituent pseudocubic cells. The body diagonal [111] is shown as a blue line. A, B, C are three oxygen ions which lie in a plane normal to the [111]. $\mathrm{O}$ is the intersection point of this plane with the [111]. In an ideal cubic symmetry, oxygen ions are located in the mid point of the side of the cube, such as the position marked as $\mathrm{D}$. The rotation of $\mathrm{BO}_{6}$ octahedron around [111], $\omega$, thus corresponds to the angle $\angle \mathrm{AOD}$. For a given oxygen displacement value $x$, (i) shows as $\alpha>60^{\circ}$, (ii) $\alpha^{\sim} 60^{\circ}$ and (iii) $\alpha<60^{\circ}$. To maintain the regular shape of the octahedron, for a nontrivial oxygen displacement $x-(-0.25) \neq 0, \alpha$ has to be smaller than $60^{\circ}$. Otherwise, a compressed stress is applied along the trigonal axis shown with the orange arrows. 
octahedron, however, has a direct influence on the states of Co ions. When the lattice symmetry is lowered from $\operatorname{Pm} \overline{3} \mathrm{~m}$ to $\mathrm{R} \overline{3} \mathrm{c}$, correspondingly the symmetry of $\mathrm{BO}_{6 / 2}$ octahedron is lowered from $O_{h}$ to $D_{3 d}$. It is found out that the allowed deformation by $D_{3 d}$ is related to $\alpha$. Megaw and Darlington [55] have defined this deformation as the octahedral strain, $\varsigma$, which corresponds to a homogeneous compression or elongation along the trigonal axis.

This effect is depicted in Fig. 6.3, which shows how for a given oxygen displacement, $x$, at a constant rotation angle, $\omega$, the shape of the octahedron changes with $\alpha$. Assuming a regular octahedral shape, $\alpha$ can be calculated based on $\omega$ : $(\cos \alpha)_{c a l}=\frac{1}{2}+\frac{\sin ^{2} \omega}{3-\sin ^{2} \omega} \geq \frac{1}{2}[55]$, indicating that as long as there is a nontrivial rotation of $\omega, \alpha$ should always be less than $60^{\circ}$ as shown in Fig. 6.3 (iii). This is opposite to what has been observed in $\mathrm{LaCoO}_{3}$ and its doped compounds, for which $\alpha$ is larger than $60^{\circ}$. For $\alpha$ larger than $60^{\circ}, \varsigma$ is negative and corresponds to a compression deformation as shown in Fig. 6.3 (i) and (ii).

Actually, this deformation, $\varsigma$, is related to the parameters of $\omega$ and $\alpha$ as follows:

$$
\varsigma=\frac{3}{2}\left[\sin \frac{\sqrt{3} \pi}{360^{\circ}}\left(60^{\circ}-\alpha\right)-\frac{\sin ^{2} \omega}{3-2 \sin ^{2} \omega}\right]\left(1-\frac{2}{3} \sin ^{2} \omega\right)
$$

The inset at the upper corner of Fig. 6.4 is the plot of $\varsigma$ in the $\omega-\alpha$ phase space, which is a curved plane. Setting the parent compound of $\mathrm{LaCoO}_{3}$ as the origin, shown as a black symbol in this plane, the change in the $\omega$ and $\alpha$ values after doping will create a specific path on the curved plane. The red lines are examples of the case 
where both $\omega$ and $\alpha$ increase or decrease such as in $\mathrm{Sr} / \mathrm{Ba}$ or $\mathrm{Ni}$ doping. ( $\omega$ increases but $\alpha$ decreases towards $60^{\circ}$ with Fe doping). Based on our refinement results, we plot $\varsigma$ as a function of $\omega$ for $\mathrm{LaCoO}_{3}(\# 1), \mathrm{La}_{1-x} \mathrm{Ba}_{x} \mathrm{CoO}_{3}$ (\#2 to 6), $\mathrm{LaCo}_{1-y} \mathrm{Ni}_{y} \mathrm{O}_{3}$ (\#8 and 9) and $\mathrm{LaCo}_{1-y} \mathrm{Fe}_{y} \mathrm{O}_{3}$ (\#10 and 11). The black line is an empirical fitting of the Ba doped samples for the R $\overline{3}$ c symmetry [55], which is the projection of curve $\varsigma(\omega, \alpha)$ on $\omega$. We can see that as the doping concentration increases, Ba quickly 'unwinds' $\omega$ towards $0^{\circ}$ and at the same time the deformation of octahedron is released ( $\varsigma$ goes towards zero). In other words, the octahedron not only rotates back, but also its shape becomes closer to the cubic point symmetry, $O_{h}$. In contrast, the effect with $\mathrm{Ni}$ doping is completely reversed: the octahedron rotates away from the midpoint of the cubic side, and also it is suppressed along [111] direction. With Fe, however, though the octahedral rotation is even slightly larger than with $\mathrm{Ni}$, the deformation remains predominantly unchanged (the inset at the bottom corner of Fig. 6.4).

Therefore, the substitution of $\mathrm{Ni}$ or Fe does not reduce the trigonal distortion. On the contrary, it is enhanced in a different manner. Fe doping increases the octahedral rotation but keeps its shape primarily unchanged, but Ni doping not only rotates the octahedron, but also compresses it.

Both manners, i.e., the rotation and the compression of the octahedron, are allowed by the trigonal distortion. The PDF study further confirmed that there is no local lattice distortion either. Fig. 6.5 (a) is the $\rho(r)$ for $y=0.40, \mathrm{Ni}^{-\mathrm{LaCoO}_{3}}$ as a function of temperature. The shape of the PDF peaks do not change but become 


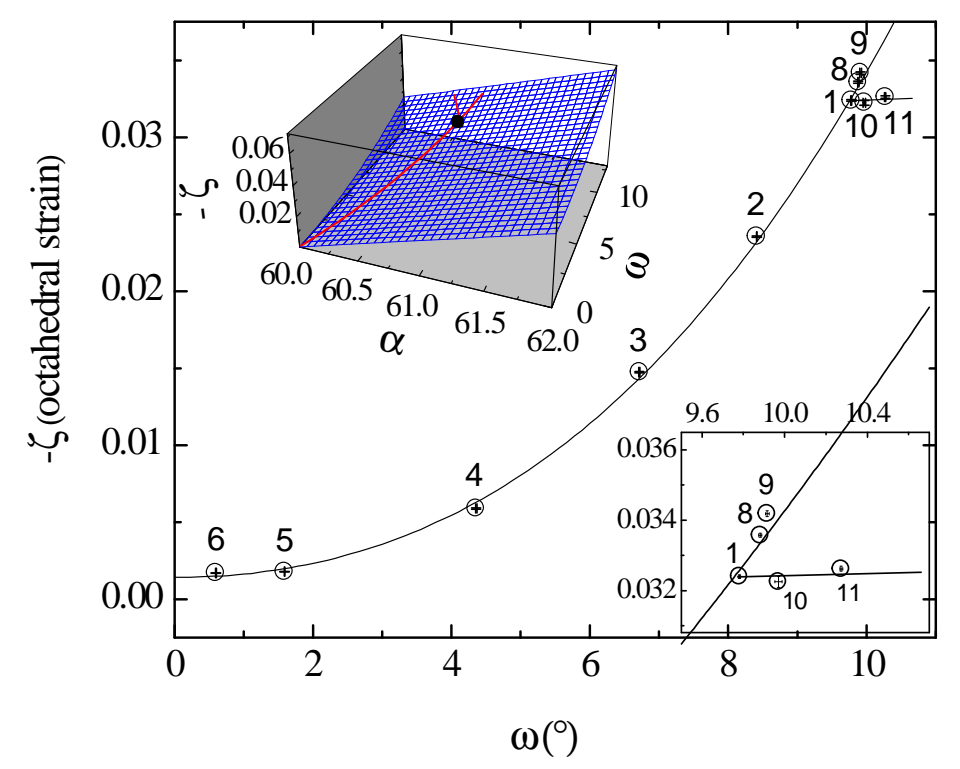

Figure 6.4: The octahedron strain $\varsigma$ as a function of $\omega$ for $\mathrm{LaCoO}_{3}(\# 1)$; $\mathrm{La}_{1-x} \mathrm{Ba}_{x} \mathrm{CoO}_{3}$ (\#2 to 6 for $x=0.1$ to 0.5 ); $\mathrm{LaCo}_{1-y} \mathrm{Ni}_{y} \mathrm{O}_{3}$ (\#8 and 9 for $x=0.1$ and 0.4); and $\mathrm{LaCo}_{1-y} \mathrm{Fe}_{y} \mathrm{O}_{3}$ (\#10 and 11 for $y=0.1$ and 0.4 ). The black line is an empirical fitting for $\mathrm{Ba}$ doped samples. The inset at the upper corner is a function of $\varsigma$ in the space of $\alpha \geq 60^{\circ}$ and $\omega \geq 0^{\circ}$. It appears as a slanted curved plane with a lowest point at $\varsigma=0$ when $\alpha=60^{\circ}$ and $\omega=0^{\circ}$. The black dot is where $\mathrm{LaCoO}_{3}$ is located on this plane and the red lines are possible paths for the cases where $\alpha$ and $\omega$ change in a same manner. The inset at the lower corner is a blow up of the high $\omega$ region. The uncertainty is shown by error bars. 


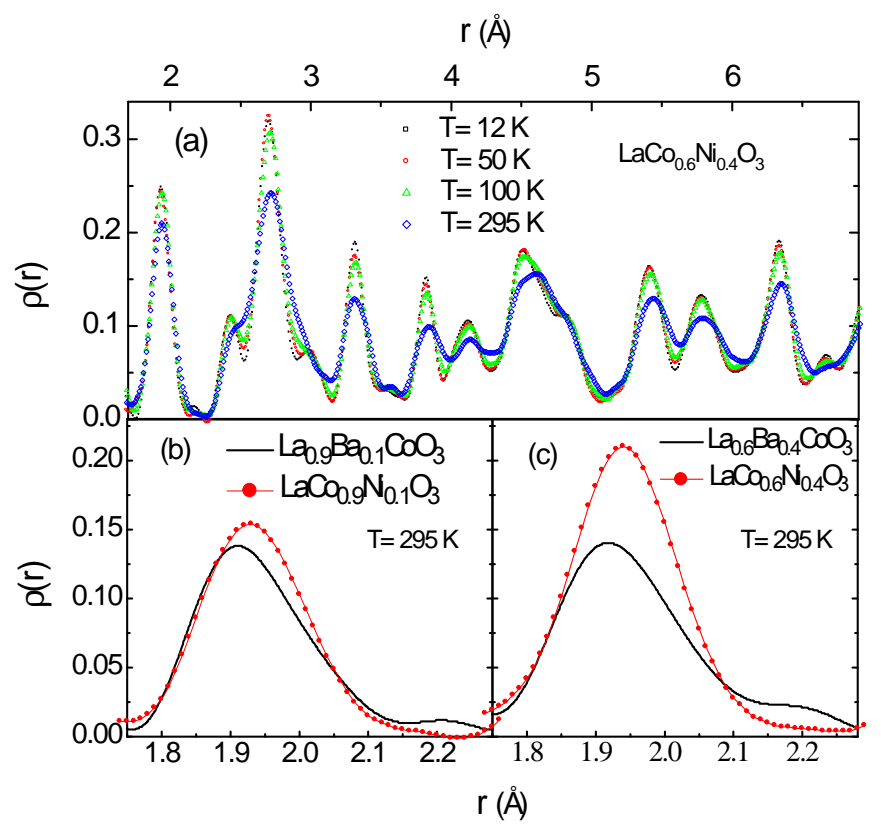

Figure 6.5: (a) The PDF of $\mathrm{LaCo}_{0.6} \mathrm{Ni}_{0.4} \mathrm{O}_{3}$ at $\mathrm{T}=12,50,100,295 \mathrm{~K}$. (b) and (c) are the comparison of the first PDF peak between $\mathrm{LaCo}_{1-y} \mathrm{Ni}_{y} \mathrm{O}_{3}$ and $\mathrm{La}_{1-x} \mathrm{Ba}_{x} \mathrm{CoO}_{3}$ for $\mathrm{x}=\mathrm{y}=10 \%$ and $40 \%$ at $295 \mathrm{~K}$, respectively. 
consistently broader as the temperature rises, and by room temperature, the peak centers slightly shift to larger $r$ values due to the lattice expansion. This indicates that the change of the lattice with temperature is dominated by the thermal vibrations of atoms around their equilibrium positions. The first peak corresponds to the transition metal and oxygen bond lengths within one octahedron and it does not overlap with any other bond length. This peak is symmetric at all temperatures. The absence of a split or asymmetry of the first peak is a strong indication for the absence of JT distortions in response to, or as a consequence of, the activation to the IS state. Similar observations have been made in the Fe doped compounds that are not shown here. This is in strong contrast to the hole doped compounds, and the comparison is made in Fig. 6.5 (b) and (c). As it can be seen, the PDF region encompassing the first peak for $\mathrm{LaCo}_{1-y} \mathrm{Ni}_{y} \mathrm{O}_{3}$ is quite different compared to $\mathrm{La}_{1-x} \mathrm{Ba}_{x} \mathrm{CoO}_{3}$ at room temperature at both concentrations, $y=0.10$ and 0.40 . In the case of the $\mathrm{Ni}$ doped compounds, this peak is clearly symmetric, indicating a single Co-O bond length which is compatible with the $\mathrm{R} \overline{3} \mathrm{c}$ symmetry. However, in $\mathrm{La}_{1-x} \mathrm{Ba}_{x} \mathrm{CoO}_{3}$, the Co-O peak shape is asymmetric and is best fit by two Gaussian peaks indicating the presence of two distinct Co-O bonds [63]. This split was suggested to be caused by the Jahn-Teller distortion which broke the $e_{g}$ and $t_{2 g}$ orbital degeneracy, in response to or as a consequence of the IS state.

It is worth noting that this JT local lattice distortion occurs when Sr or Ba doping brings the overall lattice towards the cubic symmetry which is associated with the 
regular octahedral crystal field. In fact it was reported that the percent of JT sites increases with the hole concentration; in other words, the population of the JT sites increases with the tendency of the overall structure to be cubic [63, 64].

For $\mathrm{LaCo}_{1-y} \mathrm{Ni}_{y} \mathrm{O}_{3}$ and $\mathrm{LaCo}_{1-y} \mathrm{Fe}_{y} \mathrm{O}_{3}$, unlike hole doping which released the trigonal distortion, doping $\mathrm{Ni}$ or Fe increased the trigonal distortion. Under such circumstances, Ni doping superimposed an increased 'skewed' compression onto the cooperative $\mathrm{BO}_{6 / 2}$ rotations, but $\mathrm{Fe}$ doping only increases the rotations. It is the compression that changes the shape of the octahedron and the crystal field symmetry which affects the spin state of $\mathrm{Co}^{3+}$ ions. Considering the suggestions from the bulk susceptibility measurements that for $\mathrm{Ni}$ doping the $\mathrm{Co}^{3+}$ ion is promoted to be magnetic but for Fe doping it is stabilized at LS state, this compression, therefore, is invoked to break the degeneracy of the magnetic $\mathrm{Co}^{3+}$ ions, while maintaining the Co-O bonds at constant length under the trigonal symmetry.

So far, to our best knowledge, the theoretical calculations about the energy-level diagram of $\mathrm{Co}^{3+}$ ions whether it includes spin-orbital coupling $[72,1]$ or the hybridization of $2 p-3 d$ orbitals $[43,65]$ they are all based on the assumption of cubic crystal field symmetry $\left(O_{h}\right)$ in stead of the real trigonal symmetry $\left(D_{3 d}\right)$. The $O_{h}$ crystal field can be a good approximation for the hole doped systems since the trigonal distortion is released by doping. However, for the opposite case where the trigonal distortion is enhanced such as in the Ni doped systems, whether or not this assumption is still applicable is not clear. It is often mentioned in the literature that "the cubic crystal 
field with a small trigonal distortion" [66], but how small is small?

Finally, why do the magnetic $\mathrm{Co}^{3+}$ ions in hole-doped system undergo a JT distortion, but the Ni-doped compounds adopt a "skewed" trigonal distortion? The answer may also lie in the difference of the actual crystal field between hole and Ni doping. In the $O_{h}$ crystal field which is applied well in hole doped system, the $d$ orbitals split to two-fold $e_{g}$ and three-fold $t_{2 g}$ levels, but in the $D_{3 d}$ crystal field which may be a better approximation for $\mathrm{LaCo}_{1-y} \mathrm{Ni}_{y} \mathrm{O}_{3}$, the three-fold $t_{2 g}$ levels further split into a single $a_{1 g}$ state and two-fold $e_{g}$ states [15]. The difference of the crystal field levels between symmetry of $O_{h}$ and $D_{3 d}$, therefore, could cause the octahedral deformation to break the degeneracy in a different way.

\subsection{Absence of AFM in Spin-Glass State}

Though $\mathrm{LaCo}_{1-y} \mathrm{Ni}_{y} \mathrm{O}_{3}$ shows similarity in the bulk magnetic properties as $\mathrm{La}_{1-x}(\mathrm{Sr} / \mathrm{Ba})_{x} \mathrm{CoO}_{3}$, i.e., both systems develop to the $\mathrm{SG}$ state and then FM state with increased doping, our investigations on the magnetic correlations in the SG state of $\mathrm{LaCo}_{1-y} \mathrm{Ni}_{y} \mathrm{O}_{3}$ via elastic neutron scattering, however, only identified FM correlations and no second magnetic correlations [96]. The elastic neutron-scattering measurements on the single crystal of $\mathrm{LaCo}_{0.9} \mathrm{Ni}_{0.1} \mathrm{O}_{3}$ were performed at the SPINS cold neutron triple-axis spectrometer at the NIST Center for Neutron Research. The experimental setup configuration was as follows: an $E_{f}=3.6 \mathrm{meV}$ was used with 80' -80 ' collimators before and after the sample. A Be and cooled BeO filter were used 
before and after the sample, respectively. Three flat pyrolytic graphite blades were used as the analyzer. For the purpose of crystal alignment, the cubic notation was used and the measurements were carried out in the $(h h l)$ plane.

Fig. 6.6 (a) shows an elastic neutron scattering scan in the $(h h l)$ plane along the $[h, h, 1-|h|]$ direction for the $y=0.10, \mathrm{Ni}$ doped single crystal at two temperatures, $\mathrm{T}=5$ and $80 \mathrm{~K}$. The inset is the order parameter of the FM intensity compared to the field-cooled bulk magnetization. It is clear from that no AFM correlations, either commensurate or incommensurate, are present in the spin-glass phase of $\mathrm{Ni}$ doped compositions. The increase in the intensity from the magnetic scattering at low temperatures only appears around the (001) Bragg peak, that corresponds to the FM point, and nowhere else. The broad intensity at low temperatures indicates the presence of short-range FM coupling and the estimated FM correlation length is $\sim 20$ A. A detailed contour map (Fig. 6.6 (b)) confirms the absence of magnetic superlattice reflections as the ones found in hole doped $\mathrm{Ba}$ and $\mathrm{Sr}$ samples [95, 62]. The thermal elastic neutron scattering was measured by another group [41] in the $(h k k)$ plane which also showed broadening of the (001) Bragg peak in the same composition but no AFM correlations or other magnetic superlattice structures.

It was found that the absence of the AFM correlations in $\mathrm{LaCo}_{1-y} \mathrm{Ni}_{y} \mathrm{O}_{3}$ can be understood with the same mechanism that was suggested for the hole doped compositions (Chap 5.4): the magnetic separation is coupled with the local lattice inhomogeneity. Similar to Table 5.1, the following Table 6.2 also list the average tolerance 


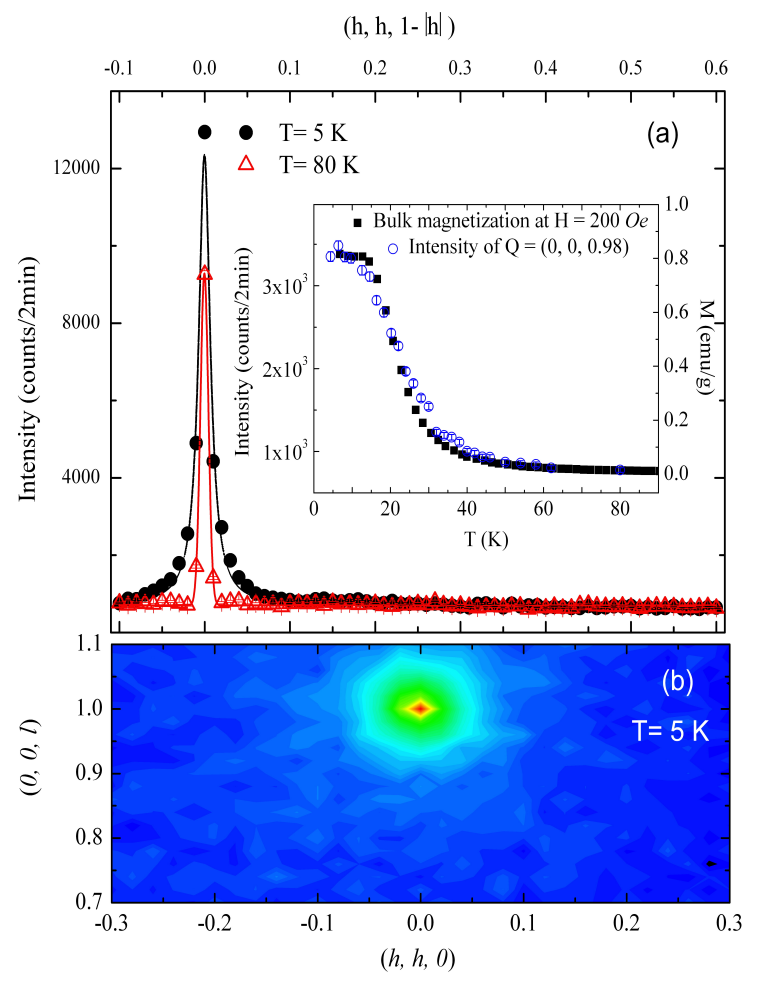

Figure 6.6: The neutron elastic scattering along the direction of $(h, h, 1-|h|)$ for $\mathrm{LaCo}_{0.9} \mathrm{Ni}_{0.1} \mathrm{O}_{3}$ single crystal at $\mathrm{T}=5$ and $80 \mathrm{~K}$. A broad intensity due to magnetic scattering at low temperature only appears around the (001) Bragg peak, indicating the presence of short-range ferromagnetic correlations. The inset shows the ferromagnetic order parameter compared with field-cooled bulk magnetization. (b) is an elastic contour map of the $(h h l)$ plane. 


\begin{tabular}{lcccc}
\hline \hline & $\mathbf{t}_{\text {avg }}$ & $\mathbf{t}_{\text {dopant-rich }}$ & $\%$ enhancement & AFM feature \\
\hline \hline $\mathrm{Ba}^{2+}, \mathrm{Co}^{4+}, \mathrm{O}^{2-}$ & 0.9653 & 1.061 & $10.867 \%$ & short-ICM to long-AFM \\
$\mathrm{Sr}^{2+}, \mathrm{Co}^{4+}, \mathrm{O}^{2-}$ & 0.9662 & 1.000 & $4.493 \%$ & short-range ICM \\
$\mathrm{Ca}^{2+}, \mathrm{Co}^{4+}, \mathrm{O}^{2-}$ & 0.9582 & 0.952 & $-0.522 \%$ & no AFM \\
$\mathrm{La}^{3+}, \mathrm{Ni}^{3+}, \mathrm{O}^{2-}$ & 0.9578 & 0.950 & $-0.731 \%$ & no AFM \\
$\mathrm{La}^{3+}, \mathrm{Fe}^{3+}, \mathrm{O}^{2-}$ & 0.9576 & 0.909 & $-5.016 \%$ & no AFM \\
\hline \hline
\end{tabular}

Table 6.2: A continued Table after table 4.1. The data of Ni and Fe doped systems are also included

factor, $\mathbf{t}_{\text {avg }}$ and the local tolerance factor of dopant rich region, $t_{\text {dopant-rich }}$, etc., for $\mathrm{Ni}$ and Fe doped systems. It can be seen that like Ca doping, where the enhancement is small, i.e. the difference between the two tolerance factors are small and the values appear to be negative, the AFM correlations are absent. With Fe doping, however, a large negative \% enhancement is observed, but consider that $10 \% \mathrm{Fe}$ is still paramagnetic and becomes AFM only above $40 \%$. The different magnetism with Fe doping compared to $\mathrm{Ni}$ and hole doping is because $\mathrm{Co}^{3+}$ is in the low spin configuration and the $\mathrm{AFM}$ order in $\mathrm{LaCo}_{1-y} \mathrm{Fe}_{y} \mathrm{O}_{3}$ is caused by $\mathrm{Fe}^{3+}$, not $\mathrm{Co}^{3+}$.

Therefore, like the case in $\mathrm{La}_{1-x} \mathrm{Ca}_{x} \mathrm{CoO}_{3}$, the absence of AFM magnetic phase in $\mathrm{LaCo}_{1-y} \mathrm{Ni}_{y} \mathrm{O}_{3}$ corresponds to the absence of the lattice inhomogeneity due to the negligible ionic radius difference between $\mathrm{Ca}^{2+}$ and $\mathrm{La}^{3+}$, and $\mathrm{Ni}^{3+}$ and $\mathrm{Co}^{3+}$ respectively. 


\subsection{Dilute Doped $\mathrm{LaCo}_{1-y} \mathrm{Ni}_{y} \mathrm{O}_{3}(y \leq 0.001)$}

$\mathrm{Fe}$ and $\mathrm{Ni}$ are both the closest neighbors of $\mathrm{Co}$ on the periodic table, but from the discussion in the previous sections, it is obvious that doping a moderate amount of $\mathrm{Fe}$ and $\mathrm{Ni}$ on the Co site (10\% to $40 \%)$ has essentially different consequences on the magnetic and transport properties. This was found to be true even when the amount of $\mathrm{Ni}$ and Fe dopant are so tiny $(y \leq 1 \%)$ that the compounds are still in the paramagnetic state [97].

\subsubsection{Bulk Properties of Dilute Doped $\mathrm{LaCo}_{1-y} \mathrm{Ni}_{y} \mathrm{O}_{3}$}

- Bulk magnetization

The bulk magnetizations as a function of temperature, $\chi(T)$, were measured on the Physical Property Measurement System (PPMS) on the powder samples of $\mathrm{LaCo}_{1-y} \mathrm{~B}_{y} \mathrm{O}_{3}(\mathrm{~B}=\mathrm{Fe}, \mathrm{Ni}, y=0.005,0.01)$ under the field of 1000 Oe. Fig. 6.7 (a) shows the $\chi(T)$ data of lightly doped compounds, $\mathrm{LaCo}_{0.995} \mathrm{Ni}_{0.005} \mathrm{O}_{3}$ (data in red open square), $\mathrm{LaCo}_{0.99} \mathrm{Ni}_{0.01} \mathrm{O}_{3}$ (data in red close square) and $\mathrm{LaCo}_{0.995} \mathrm{Fe}_{0.005} \mathrm{O}_{3}$ (blue open triangle), compared with the parent compound $\mathrm{LaCoO}_{3}$ (black close circle) which was measured under the field of 10,000 Oe. It can be seen that at the same concentration the increase of $\chi$ from $\mathrm{T} \sim 30$ to $100 \mathrm{~K}$ due to the thermal spin state transition is suppressed in Ni sample more than in Fe. The Curie-like behavior below $30 \mathrm{~K}$ is mainly attributed to the surface cobalt for the pure sample [93, 76], 


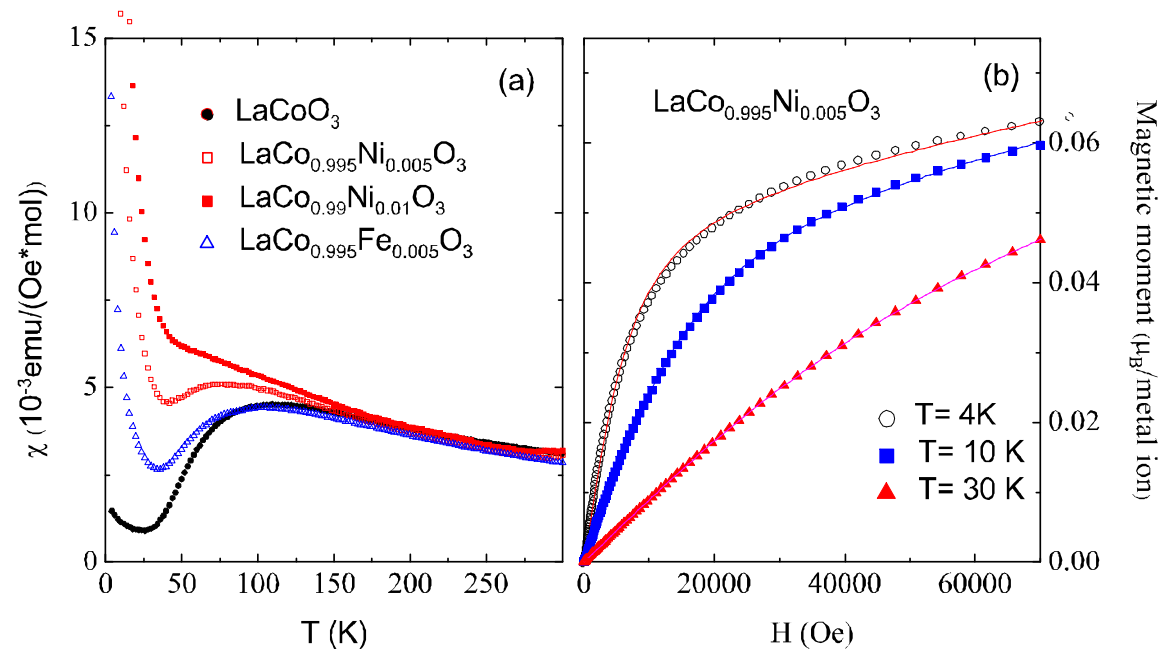

Figure 6.7: (a) Temperature dependence of the magnetic susceptibility for $\mathrm{LaCo}_{1-y} \mathrm{~B}_{y} \mathrm{O}_{3}(\mathrm{y}=0,0.005,0.01, \mathrm{~B}=\mathrm{Ni}$ and $\mathrm{Fe})$. (b) The field dependence of the magnetization for $\mathrm{LaCo}_{0.995} \mathrm{Ni}_{0.005} \mathrm{O}_{3}$ at $\mathrm{T}=4,10$ and $30 \mathrm{~K}$. The lines are the fitting with the modified Brillouin function.

but for the doped samples it also contains the contribution from the magnetic dopant $\mathrm{Ni}^{3+}$ and $\mathrm{Fe}^{3+}$ ions. Ni doping exhibits a stronger Curie-like feature than Fe which is counter intuitive, considering $\mathrm{Fe}^{3+}$ has a larger spin than $\mathrm{Ni}^{3+}$ in the perovskite structure $\left(S_{F e^{3+}}=\frac{5}{2}\right.$ vs. $\left.S_{N i^{3+}}=\frac{1}{2}\right)$.

In Fig. 6.7 (b) the field dependence of the magnetization was shown for the single crystal $\mathrm{LaCo}_{0.995} \mathrm{Ni}_{0.005} \mathrm{O}_{3}$ at low temperatures where the Curie-Weiss paramagnetic behavior dominates. The data was fitted by the summation of the Brillouin function $B_{S}(y)$ and a $H$-linear term: $M(H)=n g_{s} S B_{S}(y) \mu_{B}+\chi_{0} H \mu_{B}\left(y=\frac{\mu_{0} g_{s} S \mu_{B} H}{K_{B} T}\right)$, where $n$ is the calculated doping concentration; $S$ is the effective spin value per $\mathrm{Ni}^{3+}$ dopant; and $\chi_{0}$ is the coefficient of $H$-linear term. Table 6.3 listed the results of these parameters from the best fitting. It can be seen that the calculated doping concen- 


\begin{tabular}{cccc}
\hline \hline $\mathrm{T}(\mathrm{K})$ & $n(/$ metal ion $)$ & $g_{s} S\left(\mu_{B} / \mathrm{Ni}\right.$ ion $)$ & $\chi_{o}\left(\mu_{B} /\right.$ metal ion $\left./ O e\right)$ \\
\hline 4 & 0.0026 & 19.11 & $2.03 \times 10^{-7}$ \\
10 & 0.0024 & 20.60 & $1.91 \times 10^{-7}$ \\
30 & 0.0020 & 20.01 & $2.41 \times 10^{-7}$ \\
\hline \hline
\end{tabular}

Table 6.3: The summary of the best fitted parameters for the magnetic dependence of $\mathrm{LaCo}_{0.995} \mathrm{Ni}_{0.005} \mathrm{O}_{3}$.

tration, $n \sim 0.002$, is lower than the nominal value, $y=0.005$, which may be due to offstoichiometry. More importantly, the value of $g_{s} S$ is already as large as $\sim 20 \mu_{B}$ per $\mathrm{Ni}$ ion when the temperature is below $30 \mathrm{~K}$. Such a large magnetization value cannot be explained by only considering the contribution from $\mathrm{Ni}^{3+}$ ion. Thus, the field dependence data suggest that some of the $\mathrm{Co}^{3+}$ ions are magnetic at the low temperatures.

- Bulk electric resistivity

In hole doped systems, the FM and IMT happen at the same doping concentration. The coupled FM-IMT was well understood within the framework of DE mechanism, where the percolation of the FM-metallic clusters leads to both transitions. However, in $\mathrm{LaCo}_{1-y} \mathrm{Ni}_{y} \mathrm{O}_{3}$, although doping $\mathrm{Ni}$ brings the system into the FM state at $y \sim$ $10-15 \%$, the IMT comes in at a much higher $\mathrm{Ni}$ concentration of $\sim 40 \%$. On the insulating side, the electrical resistivity data obtained from the powder samples at range of $y=2 \%$ to $45 \%$ were found to be fitted very well with the form for Mott variable range hopping $(\mathrm{VRH})$, and the IMT was classified to be the conventional Mott-Anderson type [29], which is a common type for extrinsic semiconductors. 
Therefore, for cobaltites the percolation type of IMT shows a much higher efficiency at translating a Mott insulator into a metal than the conventional MottAnderson type. The resistivity measurements of the lightly doped compounds $\left(\mathrm{LaCo}_{1-y} \mathrm{Ni}_{y} \mathrm{O}_{3}, y \sim 0.5 \%\right.$ and $\left.1 \%\right)$ showed very interesting results, which may provide important insight on the origin of the different doping effects on the conductivity with hole and Ni.

Fig. 6.8 is the temperature dependence of the bulk electrical resistivity measured on the single crystal of $\mathrm{LaCo}_{0.995} \mathrm{Ni}_{0.005} \mathrm{O}_{3}$ (upper panel) and $\mathrm{LaCo}_{0.99} \mathrm{Ni}_{0.01} \mathrm{O}_{3}$ (lower panel). Each sample was also measured under an applied magnetic field from 3 to 7 T. The $d c$ resistivity measurements were made on PPMS using a standard four-probe technique on the single crystal samples. First, it is apparent that the data have little field dependence. Second, it is noticeable that though the temperature dependences of both samples roughly follow typical insulator behavior, i.e., as $\mathrm{T}$ is lowered the resistivity decreases, a kink occurred in $\mathrm{LaCo}_{0.995} \mathrm{Ni}_{0.005} \mathrm{O}_{3}$ and in $\mathrm{LaCo}_{0.99} \mathrm{Ni}_{0.01} \mathrm{O}_{3}$ it became more obvious and appeared as a broad bump.

Note that the temperatures of the abnormal increase of resistivity in both compositions are $\sim 100 \mathrm{~K}$, which is the same temperature as the thermal spin-state transitions as seen from the bulk magnetic susceptibility (Fig. 6.7). Therefore, the occurrence of the magnetic $\mathrm{Co}^{3+}$ appeared to hinder the conductivity.

To understand this anomaly, it is important to know the main contribution to the conductance. It is known that pure $\mathrm{LaNiO}_{3}$ is a good metal with $\mathrm{Ni}^{3+}$ ions providing 


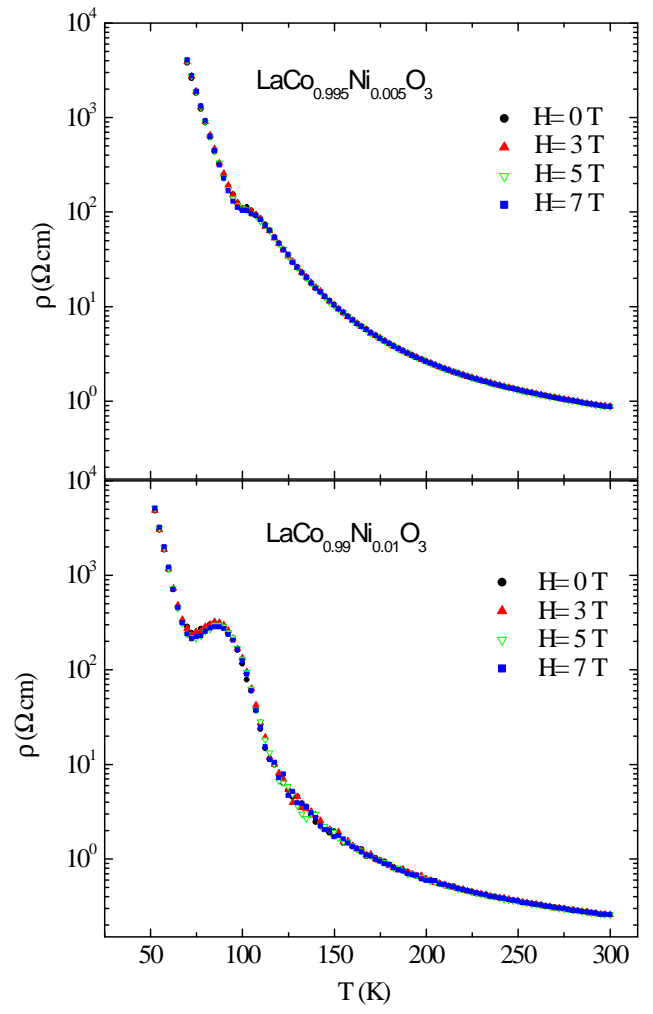

Figure 6.8: Electric resistivity as a function of temperature on the single crystal $\mathrm{LaCo}_{0.995} \mathrm{Ni}_{0.005} \mathrm{O}_{3}$ and $\mathrm{LaCo}_{0.99} \mathrm{Ni}_{0.01} \mathrm{O}_{3}$. Each sample is also measured under the magnetic field at 3,5 and $7 \mathrm{~T}$. 
$\sigma^{*}$ electrons and contributing to the conductance. Then, how does $\mathrm{Ni}^{3+}$ behave as the dopant in $\mathrm{LaCo}_{1-y} \mathrm{Ni}_{y} \mathrm{O}_{3}$ ?

The thorough studies on the nickelates $\mathrm{LnNiO}_{3}(\mathrm{Ln}=$ rare earth) [54] indicated that the mobility of the $e_{g}$ electrons of $\mathrm{Ni}^{3+}$ ions is strongly related with the lattice. It was found that except for $\mathrm{LaNiO}_{3}$, the other compounds with $\mathrm{Ln}=\mathrm{Pr}, \mathrm{Nd} \ldots$ are insulators but transfer to metals as temperature rises. The transition temperature $\mathrm{T}_{I-M}$ systematically increases with the decrease of $\mathrm{Ln}$ ionic sizes. For $\mathrm{LaNiO}_{3}$ the crystal structure is rhombohedral $(\mathrm{R} \overline{3} \mathrm{c})$ and the Ni-O-Ni bond angle is $\sim 165^{\circ}$. For $\mathrm{PrNiO}_{3}, \mathrm{NdNiO}_{3} \ldots$, the $\mathrm{Ln}$ ion is smaller and the lattice adjusts to the mismatch of $\mathrm{A}$ $\mathrm{O}$ and B-O bonds through a lowering of the symmetry to orthorhombic. This results in the Ni-O-Ni bond angle more buckled and the tendency to be more insulating. For instance, in $\mathrm{PrNiO}_{3}$ the $\mathrm{Ni}-\mathrm{O}-\mathrm{Ni}$ is $\sim 159^{\circ}$ and the $\mathrm{T}_{I-M}$ is about $130 \mathrm{~K}$, and in $\mathrm{NdNiO}_{3}$ the Ni-O-Ni is more bent to be $\sim 157^{\circ}$ and the $\mathrm{T}_{I-M}$ increases to $200 \mathrm{~K}$.

Now in $\mathrm{LaCo}_{1-y} \mathrm{Ni}_{y} \mathrm{O}_{3}$, first of all we know from Chap 6.2 that the lattice remains rhombohedral upon Ni doping. The Rietveld analysis showed that the M-O-M bond angle is $163.87^{\circ}$ for $\mathrm{LaCo}_{0.995} \mathrm{Ni}_{0.005} \mathrm{O}_{3}$ at $\mathrm{RT}$, which is almost the same as that for pure $\mathrm{LaCoO}_{3}\left(163.88^{\circ}\right)$. When the Ni concentration increases, the M-O-M bond angle slightly decreases, but it is still $163.65^{\circ}$ for $y=40 \%$ [96]. Thus, the structure of $\mathrm{LaCo}_{1-y} \mathrm{Ni}_{y} \mathrm{O}_{3}$ is surely much closer to $\mathrm{LaNiO}_{3}$ than to the other nickelates. Consequently, the $e_{g}$ electrons of the $\mathrm{Ni}^{3+}$ dopant should be highly mobile in $\mathrm{LaCo}_{1-y} \mathrm{Ni}_{y} \mathrm{O}_{3}$. 
Therefore, these highly mobile electrons most likely contribute to the electrical conductivity. In a simple classical view, the abnormal increase in the resistivity (Fig. 6.8) means that the mean-free path of the conductance electrons is decreased. Remembering the fact that the resistivity of $\mathrm{LaCo}_{1-y} \mathrm{Ni}_{y} \mathrm{O}_{3}$ on the insulating side follows VRH and that the IMT is the Anderson-Mott type, the shortening of the free paths is thus caused by the scattering from random potentials. This anomaly happens around $100 \mathrm{~K}$, and at this temperature the thermally-induced spin-state transitions reach a climax as seen from the bulk $\chi$ (Fig. 6.7). Therefore, this suggests that the random scattering can be due to the occurrence of magnetic $\mathrm{Co}^{3+}$ ions which are distributed randomly (refer to Fig.6.16 which will be also discussed later). In short, the abnormal increase in the resistivity around $100 \mathrm{~K}$ is caused by the scattering from the randomly distributed magnetic $\mathrm{Co}^{3+}$ ions.

Therefore, in $\mathrm{LaCo}_{1-y} \mathrm{Ni}_{y} \mathrm{O}_{3}$ the dopant $\mathrm{Ni}^{3+}$ and the original $\mathrm{Co}^{3+}$ sort-of act against each other for the conductance, and this is essentially different from the hole doping, where the $\mathrm{Co}^{4+}$ ions introduced by the dopant and $\mathrm{Co}^{3+}$ ions work together through DE, which establishes FM and metallicity at the same time. This can also explain why it takes significantly more Ni than holes to bring about the IMT.

On the other hand, the "anti-acting" Ni dopants with Co ions may further imply that the nature of magnetic interactions is not as simple as the DE, or if there is a FM super-exchange between $\mathrm{Ni}$ and $\mathrm{Co}$, due to the scattering from the extra electron of $\mathrm{Ni}^{3+}$, then the interaction is weak and not as strong as the one in the DE (these 
two scenarios will be discussed in Chap 6.5) .

\subsubsection{1 meV Excitation in Dilute Doped $\mathrm{LaCo}_{1-y} \mathrm{Ni}_{y} \mathrm{O}_{3}$}

A weak excitation at $\sim 1.1 \mathrm{meV}$ was first observed in lightly doped $\mathrm{LaCo}_{1-y} \mathrm{Ni}_{y} \mathrm{O}_{3}$ samples via inelastic cold neutron scattering. The measurements were conducted on DCS time-of-flight instrument at NIST on the powder samples. Each sample was about $50 \mathrm{~g}$, and the wavelength of incident neutron beam is $5 \AA$. Fig. 6.9 (a) shows the low-energy spectra at $11 \mathrm{~K}$ for $\mathrm{LaCo}_{0.995} \mathrm{Ni}_{0.005} \mathrm{O}_{3}, \mathrm{LaCo}_{0.995} \mathrm{Fe}_{0.005} \mathrm{O}_{3}$ and $\mathrm{LaCoO}_{3}$. Clearly, an excitation at $\sim 1.1 \mathrm{meV}$ only appeared in the Ni sample, and not in the Fe and the pure compound. In panel (b), the spectra were measured only on the Ni samples, but at different concentrations. This excitation appears in both $\mathrm{LaCo}_{0.995} \mathrm{Ni}_{0.005} \mathrm{O}_{3}$ and $\mathrm{LaCo}_{0.99} \mathrm{Ni}_{0.01} \mathrm{O}_{3}$, when the compositions are in the paramagnetic state, but it does not appear in $\mathrm{LaCo}_{0.9} \mathrm{Ni}_{0.1} \mathrm{O}_{3}$, which is in the $\mathrm{SG}$ state. Also, it is noticed that as $\hbar \omega \rightarrow 0$ a broad quasi-elastic intensity shows in the paramagnetic compounds even at the low temperatures but not in the SG compound. Therefore, the quasi-elastic intensity most likely corresponds to the paramagnetic scattering when the spins are not frozen in time.

Fig. 6.9 (c) shows the temperature dependence of the excitation measured on $\mathrm{LaCo}_{0.99} \mathrm{Ni}_{0.01} \mathrm{O}_{3}$. It appears the most prominent below $30 \mathrm{~K}$, and when the temperature rises above $30 \mathrm{~K}$, the excitation at $\sim 0.6 \mathrm{meV}$ and the center quasi-elastic scattering start to dominate the spectra. The $0.6 \mathrm{meV}$ excitation is due to the thermal 


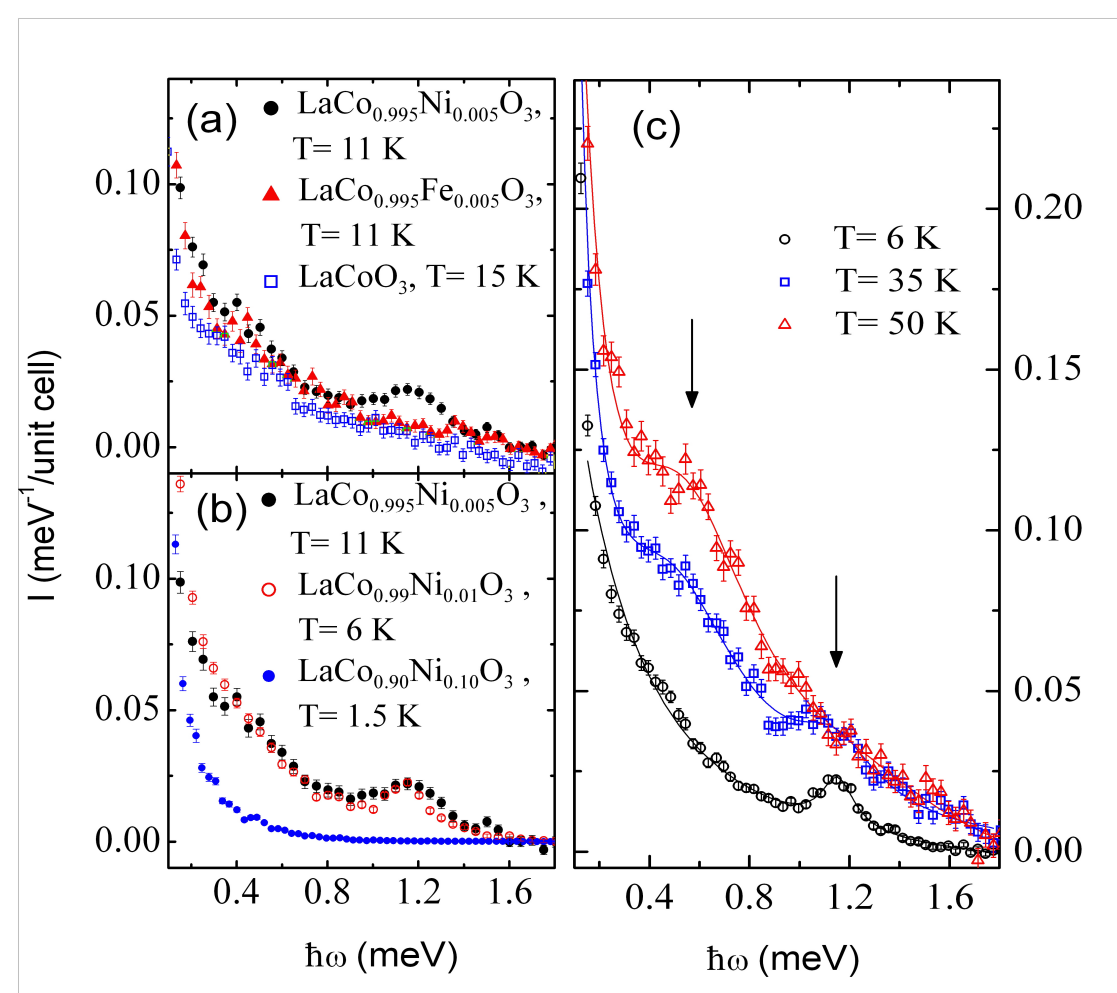

Fig. 2

Figure 6.9: (a) The low energy spectra at $11 \mathrm{~K}$ among different compositions, $\mathrm{LaCo}_{0.995} \mathrm{Ni}_{0.005} \mathrm{O}_{3}, \mathrm{LaCo}_{0.995} \mathrm{Fe}_{0.005} \mathrm{O}_{3}$ and $\mathrm{LaCoO}_{3}$. (b) The spectra for $\mathrm{LaCo}_{1-y} \mathrm{Ni}_{y} \mathrm{O}_{3}$ at different doping concentrations, $y=0.005,0.01$ and 0.1 . (c) The temperature dependence of the $1.1 \mathrm{meV}$ excitation. All data are symmetrized by the detail balance and normalized to the absolute units 
spin state transition from the $\mathrm{Co}^{3+}$ in the matrix, and apparently in the paramagnetic state the majority of the $\mathrm{Co}^{3+}$ ions still maintain their behaviors as in the pure compound.

The temperature dependent data were fit by three Lorentzian functions (solid lines in Fig. 6.9 (c)) to represent the intensities from: the center quasi-elastic scattering, the $0.6 \mathrm{meV}$ excitation, and the new excitation which is centered at $1.15 \pm 0.004$ meV. The increase in the spectra at high $\triangle \mathrm{E}$ as temperature rises mainly comes from the increased quasi-elastic intensities (Fig. 6.9 (c)). Because of its obvious temperature dependence, the $1.1 \mathrm{meV}$ excitation is treated as a magnetic signal. Its integrated intensity after subtracting the data of $\mathrm{LaCoO}_{3}$ as a background gave rise to an estimated magnetic moment [46], $g_{S} \sqrt{S(S+1)} \sim 21 \mu_{B} / N i$ ion, which is in a good agreement with the bulk magnetization data discussed in the last section.

To confirm that the $1.1 \mathrm{meV}$ excitation is magnetic in nature, the low energy spectra at $5 \mathrm{~K}$ were measured under the different magnetic fields on $\mathrm{LaCo}_{0.99} \mathrm{Ni}_{0.01} \mathrm{O}_{3}$. The measurements were performed on SPINS at NIST on an annealed powder rod about $10 \mathrm{~g}$ using a vertical field superconducting magnet. A horizontally focused analyzer with $11 \mathrm{PG}$ blades was used with fixed $E_{f}=3.7 \mathrm{meV}$; the horizontal 80' collimation was used before the sample and a radial collimator and a $\mathrm{BeO}$ filter were used after the sample .

In Fig. 6.10 as the applied field increased from 0 to 0.5 to $0.72 \mathrm{~T}$ (panel (a) to (b) to (c)), the intensity of the excitation visibly became broader and the peak 
height became shorter. By $\mathrm{H}=2 \mathrm{~T}$ (Fig. 5.10 (d)) there is hardly any visible signal; instead, the data is well fit by a constant background (the solid line) which appears slightly higher than the background under the zero field. The inset of panel $(d)$ is the intensity at a constant energy transfer, $\triangle E=1.2 \mathrm{meV}$, as a function of magnetic field up to 2 T. Clearly, the excitation responded to the weak field as the intensity decreased as the field increased. At $\mathrm{H} \sim 1 \mathrm{~T}$, the excitation diminished to be the background level.

The data under the fields showed broadening but not splitting. Therefore, they are best fit by more than two Gaussian functions. In the simplest scenario, the data were fit by three Gaussians (shown as the green solid lines; the red lines are the total envelope): one in the middle which has the same position as the one without field; the other two are located symmetrically on each side of the middle peak and due to the broadening of the overall envelope, their positions are allowed to move along the opposite directions. Based on the Zeeman effect, a given energy level is split under the applied field to $2 S+1$ levels by $\triangle E=-\mu_{B} g m B, m=S, S-1, \ldots-S$. The observed excitation corresponds to the energy displacements between the split energy levels, i.e., $\triangle(\triangle E)=-\mu_{B} g \triangle m B$. Zeeman selection rule only allows the transitions with $\triangle m=0, \pm 1$, and if the transition of $|0\rangle \rightarrow|0\rangle$ is the only choice for $\triangle m=0$ it is not allowed either. The Zeeman effect of the $0.6 \mathrm{meV}$ excitation in the pure $\mathrm{LaCoO}_{3}$, for example, only results in two split peaks with the middle one absent [66]. This is because the first excited state is a triplet and the ground state is a singlet, 


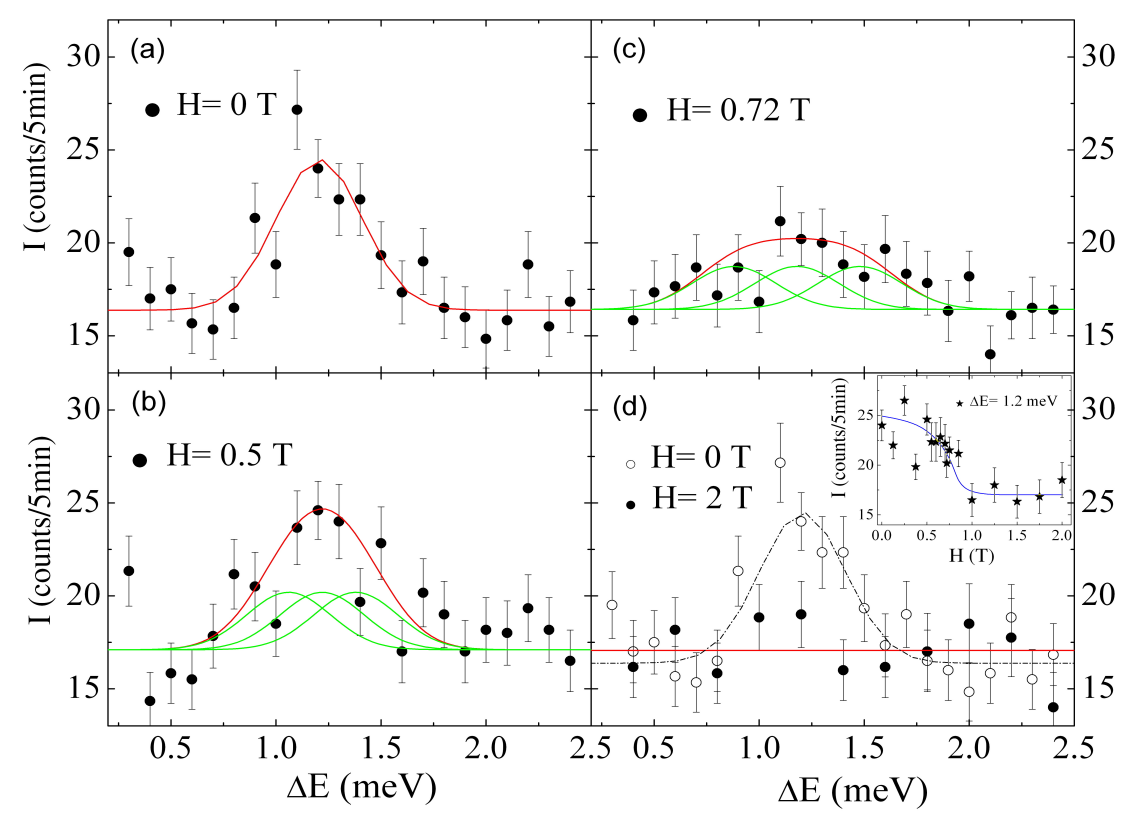

Fig. 3

Figure 6.10: The low energy spectra under the field of $0,0.5,0.72$ and $2 \mathrm{~T}$ at $5 \mathrm{~K}$ on the $\mathrm{LaCo}_{0.99} \mathrm{Ni}_{0.01} \mathrm{O}_{3}$ powder sample at $5 \mathrm{~K}$ at the constant $\mathrm{Q}$ position of $(0,0$, $1)$. The inset of $(\mathrm{d})$ is the magnetic field dependence of the excitation, measured at the constant energy transfer of $1.2 \mathrm{meV}$ at $5 \mathrm{~K}$. The green lines are the fitting with three Gaussian functions and the red line is the total profile of the fitting which is one Gaussian in (a), three Gaussians in (b) and (c) and a constant background in (d). 
and thus the zero energy displacement which corresponds to the middle peak and the transition of $|0\rangle \rightarrow|0\rangle$ are not allowed. For the $1.1 \mathrm{meV}$ excitation, however, the associated energy levels are more complicated under the presumption of the existence of the middle peak. Though the data are not sufficient to provide the absolute values of $m$, the effective $g$ value can be estimated from the broadening, which is at $\sim 11$.

Therefore, consistent with the magnetization data, the $1.1 \mathrm{meV}$ excitation also suggests the large effective $S$ or $g$ value, which strongly indicates that, even with light $\mathrm{Ni}$ doping, $\mathrm{Co}^{3+}$ ions are magnetic below $30 \mathrm{~K}$.

Finally, the other possible cause for the broadening under the field is also considered for the case of the crystal field change under the field, but under such a weak field, the associated energy change is estimated at the order of $\mu \mathrm{eV}$ which is too small to account for the data.

The inelastic neutron scattering measurements on the single crystal of $\mathrm{LaCo}_{0.995} \mathrm{Ni}_{0.005} \mathrm{O}_{3}$ further indicate there is a $\vec{Q}$ dependence associated with the $1.1 \mathrm{meV}$ excitation. Fig. 6.11 (a) and (b) are the energy scans at the constant $\vec{Q}$ position of $(0,0,1.1)$ and $(0.5,0.5,0)$, respectively. Both scans were measured at $5 \mathrm{~K}$ (data in black) and $55 \mathrm{~K}$ (data in red). It can be seen that only the energy scan at the Bragg peak position exhibits the excitation (Fig. $6.11(\mathrm{a}))$ and the energy scan at $(0.5,0.5,0)$ does not (Fig. $6.11(\mathrm{~b})$ ). As temperature increased to $55 \mathrm{~K}$, the quasi-elastic scattering significantly rose up in both $\vec{Q}$ positions. The solid lines are the overall envelope of the fitting. For the spectrum at $(0,0,1.1)$ at $5 \mathrm{~K}$ the data was fitted by two Gaussians 
which represent the centre elastic peak and the $1.1 \mathrm{meV}$ excitation, but for the one at $(0.5,0.5,0)$ only one centre Gaussian is necessary since there is no excitation; For the spectra at $55 \mathrm{~K}$, the fitting contained a centre Gaussian and a centre Lorentzian which accounted for the quasi-elastic broadening.

After the energy scans were obtained, the inelastic contours were mapped out in $(h h l)$ plane at the constant energy transfer of $1.1 \mathrm{meV}$ on MACS (Multi Axis Crystal Spectrometer) at NCNR. Fig. 6.11 (c) and (d) showed the contours at $1.5 \mathrm{~K}$ and 55 $\mathrm{K}$, respectively.

Clearly the inelastic intensities only occur around the Bragg peak positions of $(0,0,1)$ and $(0,0,0)$, and not the other positions such as $(0.5,0.5,0.5)$ or $(0.5,0.5,0)$, which suggested the existence of FM correlation and the absence of AFM. At $T=1.5$ $\mathrm{K}$, the inelastic intensity only comes from the new excitation which appears very broad and isotropic in $\vec{Q}$, indicating a short-range FM correlation only. As temperature rises to $55 \mathrm{~K}$ the pattern of the contour remained the same, but the background intensity apparently increased significantly, which is mainly attributed to the 0.6 meV excitation and the quasi-elastic scattering. Based on the discussion above, it can be seen that both $0.6 \mathrm{meV}$ excitation and the quasi-elastic intensity are broad in $\triangle E$, and hence their intensities are easily extended to $1.1 \mathrm{meV}$ and cause the lifting of the background in the inelastic channel of $1.1 \mathrm{meV}$.

Simple but interesting enough, this manner of the magnetic correlation, i.e. only FM, is maintained but "frozen" as static when the system becomes SG upon further 

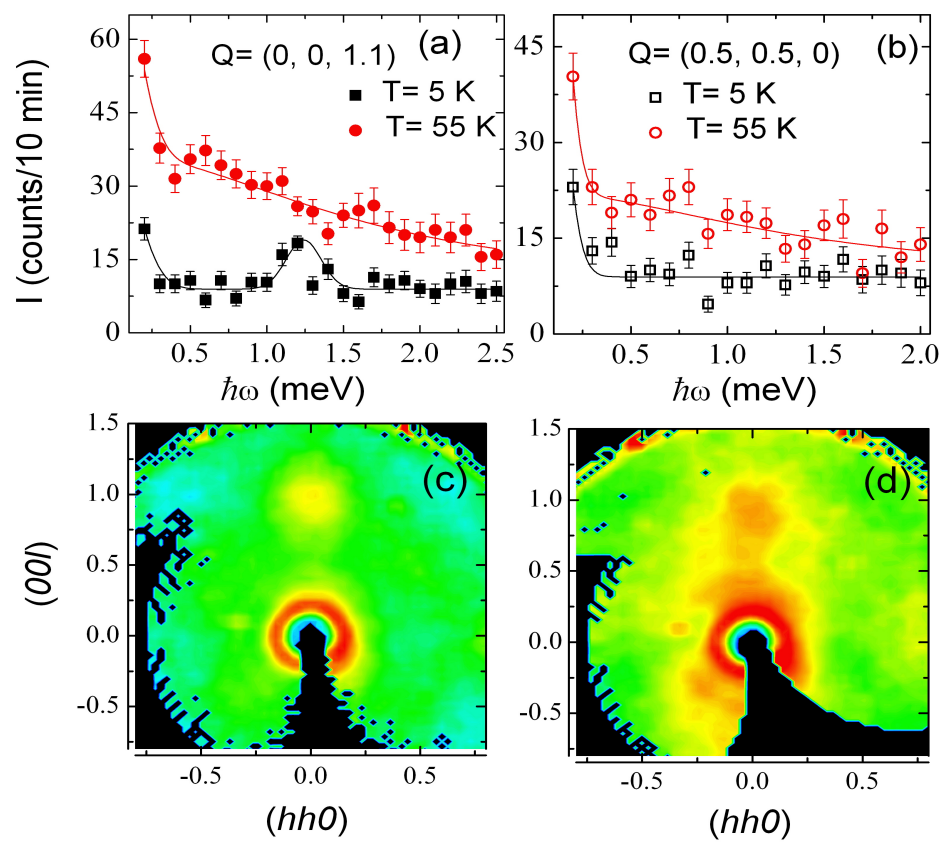

Figure 6.11: (a) and (b) are the low energy spectra on the single crystal $\mathrm{LaCO}_{0.995} \mathrm{Ni}_{0.005} \mathrm{O}_{3}$ obtained on SPINS. The energy scans were measured at the constant $\mathrm{Q}$ position of $(0,0,1.1)$ and $(0.5,0.5,0)$ at $5 \mathrm{~K}$ and $55 \mathrm{~K}$. (c) and (d) are the inelastic neutron contours obtained on MACS. They were measured at the constant energy transfer of $1.1 \mathrm{meV}$ in (hhl) plane at $1.5 \mathrm{~K}$ and $55 \mathrm{~K}$. The Q-dependence of inelastic intensities only appear around the Bragg peaks as a broad peak. The $(0,0,0)$ peak also contains the direct incident beam and its majority intensity is masked by beam stopper. 
$\mathrm{Ni}$ addition, where only short-range FM order was identified (Fig. 6.6). The AFM correlations, however, have not been ever observed.

\subsection{Magnetic Interactions in $\mathrm{LaCo}_{1-y} \mathrm{Ni}_{y} \mathrm{O}_{3}$}

Based on the magnetization and inelastic neutron scattering measurements, two conclusions can be drawn for the effects of lightly $\mathrm{Ni}$ doping: (1) the existence of magnetic $\mathrm{Co}^{3+}$ ions down to $T \rightarrow 0 \mathrm{~K}$; $(2)$ the occurrence of a weak FM coupling only.

The further search for the origin of $1.1 \mathrm{meV}$ excitation, however, may provide important insight upon the nature of the magnetic interactions.

So far the mechanism for the magnetic interactions that has been successfully applied in the cobalt oxides is super-exchange (Chap 5.2), where the FM coupling happens via $\mathrm{Co}^{3+}-\mathrm{O}^{2-}{ }_{-} \mathrm{Co}^{4+}$, and the AFM coupling via $\mathrm{Co}^{3+}-\mathrm{O}^{2-}{ }_{-} \mathrm{Co}^{3+}$. The exchange involves the interactions between the $e_{g}$ orbitals of two neighboring Co ions. This is feasible considering both ions are the same species and thus the energy levels of $e_{g}$ orbitals should be the same or very close for $\mathrm{Co}^{3+}$ and $\mathrm{Co}^{4+}$ even at the different spin states. However, in $\mathrm{Ni}$ doped system, although the experiment results indicated the existence of FM correlations, it is not clear whether the coupling necessarily occurs between $\mathrm{Co}^{3+}$ and $\mathrm{Ni}^{3+}$ ions via super-exchange or not though it seems to be a straightforward expectation because the mechanism is analogous to the DE between $\mathrm{Co}^{3+}$ and $\mathrm{Co}^{4+}$. Thus, in the following discussion, two scenarios are proposed 
regarding the nature of the magnetic interactions, based on the assumption that

(i) the energy levels of $e_{g}$ orbitals of $\mathrm{Co}^{3+}$ and $\mathrm{Ni}^{3+}$ are close enough, and thus the FM couplings are caused by the FM super-exchange via $\mathrm{Ni}^{3+}-\mathrm{O}^{2-}-\mathrm{Co}^{3+}$.

In this case the possible configurations for establishing FM coupling are demonstrated in Fig. 6.12. For simplicity, only unfulfilled orbitals are drawn. Fig. 6.12 (a) is when $\mathrm{Co}^{3+}$ is in its LS state and thus it can provide two empty $e_{g}$ orbitals. Before the $e_{g}$ electron hopped from $\mathrm{Ni}^{3+}$ the spin of the recipient ion, $\mathrm{Co}^{3+}$, is zero and after it hopped the core spin of the donating ion, $\mathrm{Ni}^{4+}$, is zero. So in this configuration, there is always one spin-zero ion and therefore the FM correlation is not able to be established.

The configuration (b) and (b') refer to the cases where the $e_{g}$ electron of $\mathrm{Ni}^{3+}$ hops to the empty $e_{g}$ orbital of the IS $\mathrm{Co}^{3+}$. Such hopping can only happen when the unpaired spins of both ions are aligned in the same direction, as shown in (b). Therefore, if the spin of $\mathrm{Ni}^{3+}$ happens to be the opposite direction then it has to flip first in order to hop, as shown in (b').

The configuration (c) and (c') described a similar situation as (b) and (b'), but in these two cases it is the $e_{g}$ electron of $\mathrm{Co}^{3+}$ hopped to the empty $e_{g}$ orbital of $\mathrm{Ni}^{3+}$.

The $1.1 \mathrm{meV}$ excitation can be the energy associated with the spin-flip.

The spin-flip process is compatible with the paramagnetic state. In the pure $\mathrm{LaCoO}_{3}$, the $0.6 \mathrm{meV}$ excitation was suggested to be the energy transfer associated with the triplet $\mathrm{Co}^{3+}(\widetilde{J}=1)$ between its manifold of $\widetilde{J}_{z}= \pm 1$ and $\widetilde{J}_{z}=0$. In a 


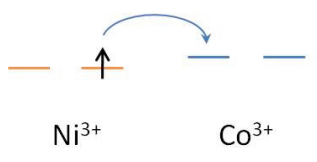

(a)

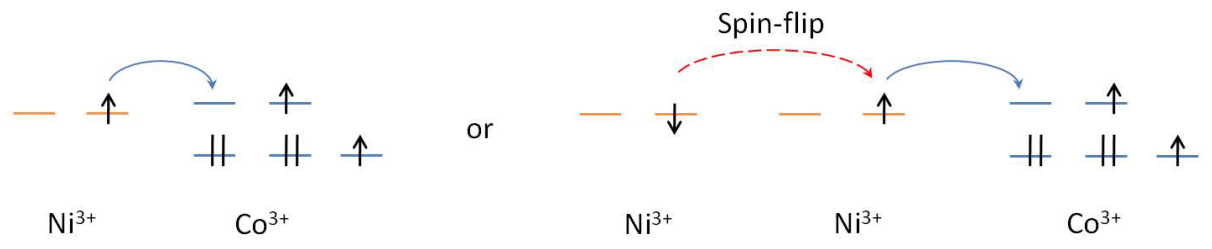

(b)

(b')

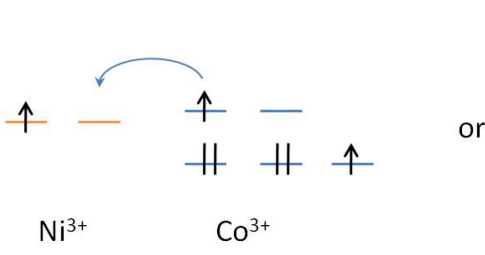

(c)

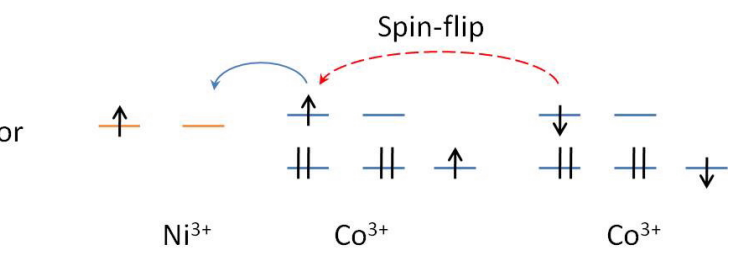

(c)

Figure 6.12: Possible configurations for the FM coupling via super-exchange between $\mathrm{Ni}^{3+}$ and $\mathrm{Co}^{3+}$ ions. 
classical picture to depict this quantum concept, the $0.6 \mathrm{meV}$ excitation corresponds to the spin-flip energy in the configuration $\left(c^{\prime}\right)$. For the $1.1 \mathrm{meV}$ excitation, first of all its value is very close to $0.6 \mathrm{meV}$ so it may share the nature as the $0.6 \mathrm{meV}$ excitation, i.e., it could be the spin-flip energy associated with the manifold of $\mathrm{Ni}^{3+}$ as shown in (b') or the one of $\mathrm{Co}^{3+}$ as shown in $\left(\mathrm{c}^{\prime}\right)$. The slightly different value from $0.6 \mathrm{meV}$ can be caused by the minor difference of the octahedral crystal field due to Ni doping.

This scenario is based on the assumption that the energy levels of $\mathrm{Ni}^{3+}$ and $\mathrm{Co}^{3+}$ are very close. Therefore this may suggest another possibility, namely that the 1.1 $\mathrm{meV}$ corresponds to the energy difference between the two $e_{g}$ orbitals. However, if this is the case it should be expected that the excitation intensity would increase with an increase in the quantity of $\mathrm{Ni}$ dopants, which is not what was observed: the excitation disappeared once the system became SG. If it is associated with the spinflip energy, then when the spins are frozen in time, i.e., not able to flip in the SG state, the excitation goes away.

Notice that for the two ions that are involved in the FM coupling, whether $\mathrm{Ni}^{3+}$ or magnetic $\mathrm{Co}^{3+}$, only one of the $e_{g}$ orbitals of each ion is empty, and the other one is always occupied. This can give rise to some small FM clusters which are confined in one-dimension (1D) or two-dimensions (2D) due to the $e_{g}$ orbitals orientation. Fig. 6.13 (a) enumerates all the possible orbital orientations for the simplest case, which is 2-site FM clusters, and (a') demonstrates several possible orientations for 4-site FM 


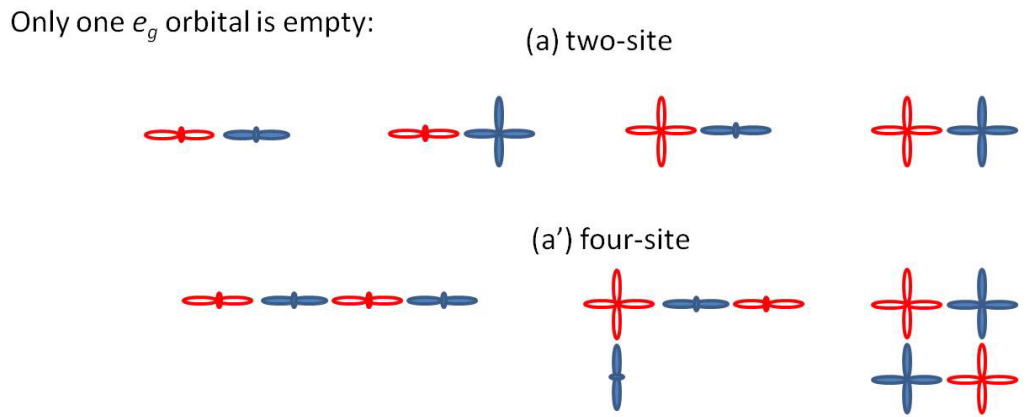

Both $e_{g}$ orbitals are empty:

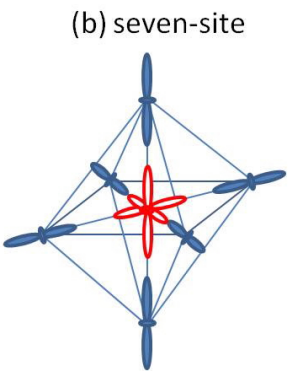

Figure 6.13: $e_{g}$ orbital orientations for several small FM clusters. The empty $e_{g}$ orbitals are drawn as red open and the occupied ones are blue solid. (a) shows the cases of two-metal-ions clusters; (a') corresponds to the case where the clusters are formed by four ions and for the recipient ion only one of the $e_{g}$ orbitals is empty. The cluster can develop along $z$ direction if the coupling empty orbital is $d_{z^{2}}$ (the first case in $\left.\left(\mathrm{a}^{\prime}\right)\right)$ or the cluster can grow in $x-y$ plane if the empty one is $d_{x^{2}-y^{2}}$ (the last two cases in $\left(\mathrm{a}^{\prime}\right)$ ). (b) shows the possbile orbital orientation in the seven-site FM cluster when the center recipient ion has both $e_{g}$ orbitals empty.

clusters in $1 \mathrm{D}$ or $2 \mathrm{D}$ (for simplicity, only metal ions' $e_{g}$ orbitals are drawn, and the oxygen ion in between is emitted. The open red orbital refers to the empty $e_{g}$, and the solid blue refers to the occupied $e_{g}$ ). If the empty orbital of the recipient ion is $d_{z^{2}}$, then the FM coupling is confined to be along the $z$ direction (the first two cases in (a) and the first case in (a')), while if the empty one is $d_{x^{2}-y^{2}}$, then the coupling with it can develop in the $x-y$ plane (the last two cases in (a')).

As a comparison, for the case that both $e_{g}$ orbitals of the recipient ion are empty, 
which can happen in the FM coupling with the $\mathrm{LS} \mathrm{Co}^{4+}$ ion, the coupling can occur in both the $z$ direction and the $x-y$ plane at the same time. Presumably, the probability is the same along all six directions $( \pm x, \pm y, \pm z)$. This results in a 7-site cluster, as it is shown in Fig. 6.13 (b). In fact, this 7 -site FM cluster model was proposed in the hole doped system, $\mathrm{La}_{1-x} \mathrm{Sr}_{x} \mathrm{CoO}_{3}$.

Based on these small FM cluster models, we can calculate the magnetic $\mathrm{S}(\vec{Q})$ in the $(h h l)$ plane to compare with the experiment data. The magnetic scattering intensity is $I_{M}=\left|F(Q) \cdot F^{\prime}(\vec{Q})\right|^{2}$ where $F(Q)$ is the magnetic form factor of the metal ions and in the lightly Ni doped compounds the value of $\mathrm{Co}^{3+}$ is used; $F^{\prime}(\vec{Q})$ is the magnetic structure factor. In the case of spin isotropy, i.e., the magnetic moments of the spins are the same value and they point along $x, y$ and $z$ directions at an equal probability, i.e. domain averaging; $F^{\prime}(\vec{Q})$ can be written as $F^{\prime}(\vec{Q}) \propto \sum_{\vec{r}} \pm e^{-i \vec{Q} \cdot \vec{r}}$ $[47]$.

$F^{\prime}(\vec{Q})$ was calculated for the FM clusters of the 2-site model up to 7-site models. Fig. 6.14 enumerates all the possible FM cluster configurations for the 2-site and 3-site models which can be a string or a corner, and for the 4-site models which can be a string, a corner in $2 \mathrm{D}$, a square and a corner in $3 \mathrm{D}$. It turned out that the models of 2-site, 3 -site and 4-site of $1 \mathrm{D}$ and $2 \mathrm{D}$ can generate the same pattern as the experiment data but the configuration of 4-site corner in 3D generated intensities at ( $h \pm 0.5, k \pm 0.5, l \pm 0.5)$ (marked by the red cross on the contour), which indicated that the configurations of the small FM clusters can not be formed in 3D 4-site. In 

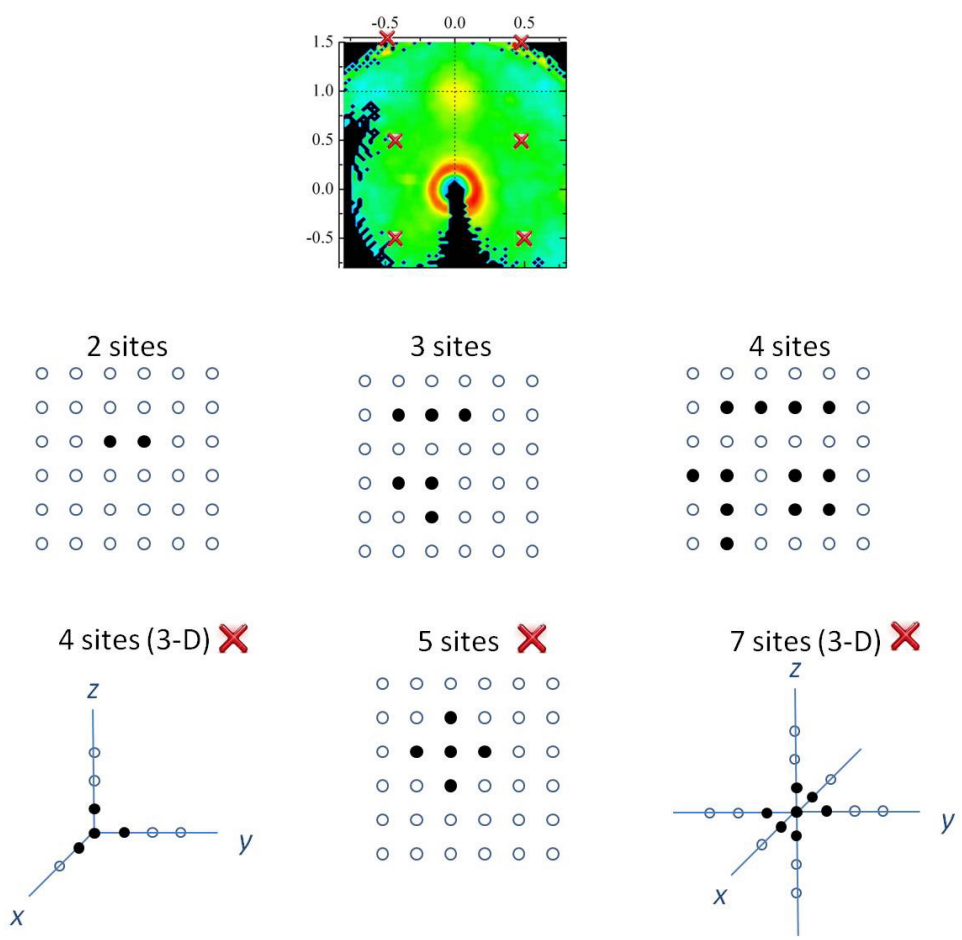

Figure 6.14: The configurations of small FM clusters for the 2-site up to 7-site models. For the 4-site model of a corner-shape in 3D and 5-site and 7-site models, the calculated magnetic intensities in (hhl) plane show peaks at the positions of $(h \pm 0.5, k \pm 0.5, l \pm 0.5)$. Therefore these models are ruled out.

addition, the models with the number of metal ions above four were also tried. It turned out that the 5 -site model in 2D (Fig. 6.14) already generated a peak at (0.5, $0.5,0.5)$ and the same for the 7-site model. In short, in order to reproduce the pattern observed in the inelastic contour (Fig. 6.11 and 6.14), the small FM clusters form only in low dimensions and with no more than 4 metal ions.

Among the models of 2-site, 3-site and 4-site of $1 \mathrm{D}$ and 2D, the best fitted ones can be determined by comparison with the calculated peak width from the experimental data. Fig. 6.15 (a) and (b) are the cuts of the inelastic contours (Fig. 6.11) along 

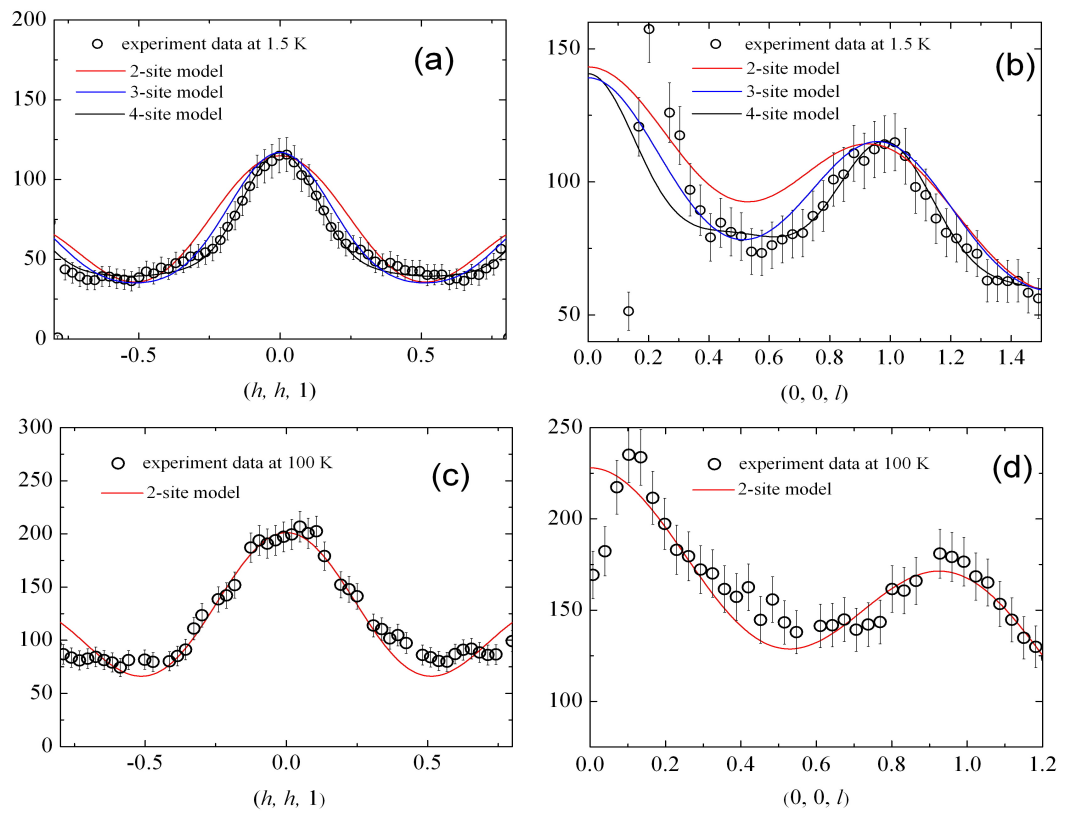

Figure 6.15: (a) and (b) are the cuts along the (hh1) and (00l) directions of the inelastic contour at $1.5 \mathrm{~K}$. The experiment data are shown as the open circles. The red solid line is the calculated peak based on the 2-site model, the blue lines and the black lines correspond to the 3-site and 4-site models, respectively. (c) and (d) are the cuts at $100 \mathrm{~K}$, compared with the 2-site model.

$(h, h, 1)$ and $(0,0, l)$ directions at $1.5 \mathrm{~K}$, and (c) and (d) are the ones at $100 \mathrm{~K}$. The open circles are the experiment data showing the broad inelastic peak at $(0,0,1)$. The red solid lines are the calculated data based on the 2-site FM cluster model, the blue lines refer to the 3 -site models and the black lines are 4-site models (the calculated intensity also takes into account the magnetic form factor of $\mathrm{Co}^{3+}$ ion). At $1.5 \mathrm{~K}$, the experimental data is best fitted by the 4-site models, and at $100 \mathrm{~K}$ the peak became so broad that 2-site model fit well. 
Finally, it should be noted that the $(0.5,0.5,0.5)$ magnetic peak is not unique for the 5-site or 7-site model discussed above. A pair of AFM cluster also reproduces this peak. In the lightly Sr-doped compounds, an magnetic excitation at $0.75 \mathrm{meV}$ was reported to have a $Q$ dependence that shows a broad peak at $(0.5,0.5,0.5)$ [67]. The data was interpreted in terms of the 7-site FM cluster only, and the possibility of AFM clusters was not considered. However, based on the discussion in Chap 5, it is known that the magnetic inhomogeneity with the existence of the AFM phase is a prominent phenomenon in the hole doped systems.

Therefore, the seemingly simple $\vec{Q}$ dependence of the 1.1 meV excitation actually expressed the uniqueness about $\mathrm{LaCo}_{1-y} \mathrm{Ni}_{y} \mathrm{O}_{3}$ : the magnetic interactions with $\mathrm{Ni}$ doping only have the FM type which starts in the paramagnetic state as a dynamic short-range correlation and as the doping increases, it grows to be longer range and static in the SG and FM state. The absence of $(0.5,0.5,0.5)$ intensity rules out the possibility of AFM correlations and its broad feature further suggested the FM clusters are small and are formed by less than five magnetic ions in 1D or 2D. Such a FM "network" certainly has a lower chance of percolation compared with the one that develops in $3 \mathrm{D}$ as in the hole doped systems. Thus this can be the reason that it takes more Ni dopants than holes to lead to the IMT.

(ii) the energy levels of $e_{g}$ orbitals of $\mathrm{Co}^{3+}$ and $\mathrm{Ni}^{3+}$ are not close enough to have super-exchange.

The 1.1 meV excitation can be associated with the excited states of two FM 
coupled spins which are not necessary the closest neighbors (Fig. 6.16). The extracted magnetic exchange constant, $J$, however, as it is discussed following is too low to be accounted by the DE, $J_{D E}$.

The Heisenberg Harmiltonian for two FM coupled spins is $H=-2 J S_{i} S_{j}$ and the eigenvalues are $E=J S_{t}\left(S_{t}+1\right)$, where $S_{t}$ is the total spin $S_{t}=\left|S_{i}+S_{j}\right|=$ $0,1, \ldots 2 S$. The observed excitation corresponds to the allowed eigenstate transitions by the selection rules, $\triangle S_{t}=0, \pm 1, \pm 2, \ldots$ For $\triangle S_{t}=1$ that is $\triangle E=2 J\left(S_{t}+1\right)=$ $2 J, 4 J, 6 J, \ldots$, corresponding to the transition of $|0\rangle \rightarrow|1\rangle,|1\rangle \rightarrow|2\rangle,|2\rangle \rightarrow|3\rangle \ldots$, respectively [23]. Thus if the excitation at $1.15 \pm 0.004 \mathrm{meV}$ corresponds to the first excitation, i.e. $|0\rangle \rightarrow|1\rangle$, it will give rise to $J=0.575 \pm 0.002 \mathrm{meV}$. Taking into account the case where the FM couplings occur among more than two spins, such as in a triad, it will result in an even smaller $J$ value.

Such a weak magnetic exchange can not be explained by the DE. Based on the spin-wave dispersion measurements via inelastic neutron scattering on $\mathrm{La}_{0.8} \mathrm{Sr}_{0.2} \mathrm{CoO}_{3}$ which is a FM, the magnetic exchange energy of DE, $J_{D E}$, is estimated to be $\sim 4$ to $8 \mathrm{meV}[73]$.

Hence, the $1.1 \mathrm{meV}$ excitation may imply that the nature of the magnetic interaction with $\mathrm{Ni}$ doping is not super-exchange. Among the few known magnetic exchange interactions, an RKKY-like or itinerant-exchange mechanism seems to be a good interpretation for such a weak interaction.

In this scenario, the highly mobile $\sigma^{*}$ electrons of $\mathrm{Ni}^{3+}\left(t_{2 g}^{* 6} \sigma^{* 1}\right)$ are localized due to 


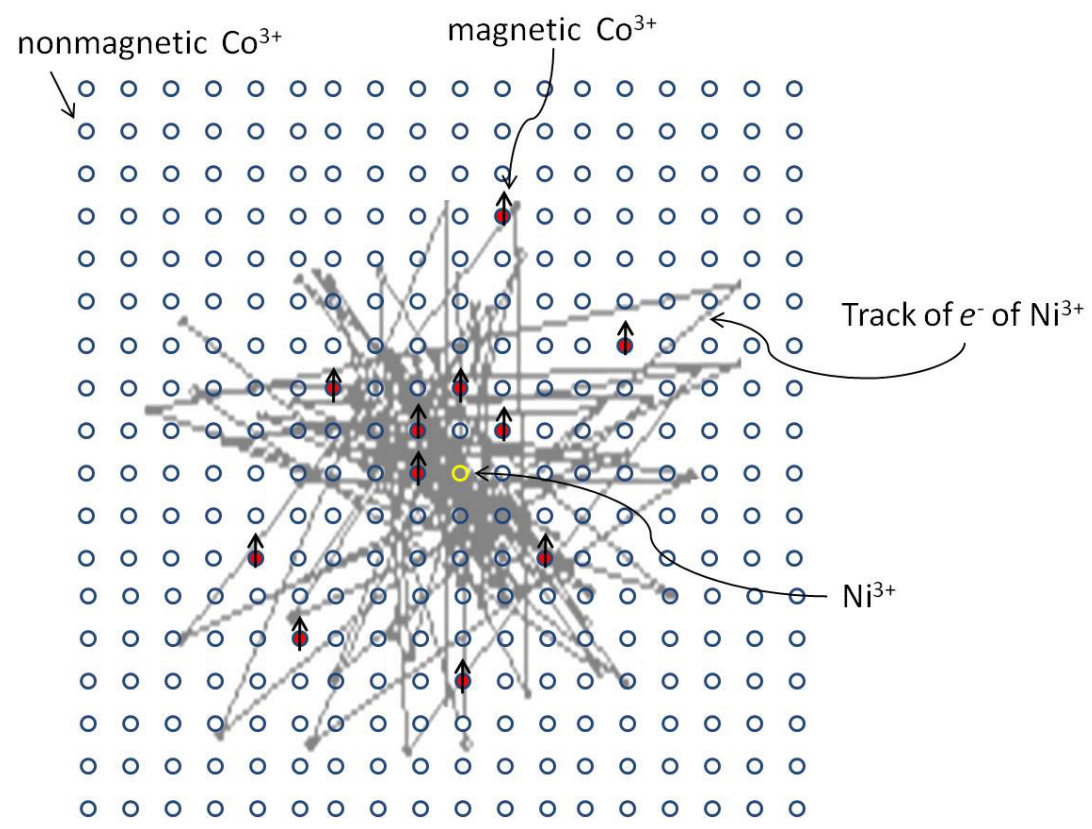

Figure 6.16: A cartoon of the Co lattice in two dimension. The blue open circles are the spin-zero $\mathrm{Co}^{3+}$ ions and the red solid circles are the magnetic ones. One $\mathrm{Ni}^{3+}$ dopant is drawn in yellow. The grey lines represent the track of the itinerate electron provided by $\mathrm{Ni}^{3+}$. Within the localization range this electron contributes to the conductivity and at the same time mediates the FM coupling among the magnetic $\mathrm{Co}^{3+}$. The scattering from the randomly distributed magnetic $\mathrm{Co}^{3+}$ on the other hand localized the electron. 
the random scattering from the magnetic $\mathrm{Co}^{3+}$ ions. Within the localization length, the $\sigma^{*}$ electron can be treated as itinerate and therefore can mediate a weak FM coupling in between the magnetic $\mathrm{Co}^{3+}$ ions. Fig. 6.16 shows a cartoon to demonstrate this mechanism. For simplicity, a two-dimensional Co lattice is shown. In lightly doped compounds, most $\mathrm{Co}^{3+}$ ions are spin-zero (blue open circles) and some of them bare spins (red close circles); one dopant $\mathrm{Ni}^{3+}$ is drawn as a yellow open circle, and when its $\sigma^{*}$ electron travels around, it is scattered by the randomly located magnetic $\mathrm{Co}^{3+}$ ions; the grey lines are meant to illustrate a classical picture of the electron "track" and how it is localized by the random scattering. This is a similar mechanism to RKKY, which typically occurs in metals. However, on the insulating side of the $\mathrm{LaCO}_{1-y} \mathrm{Ni}_{y} \mathrm{O}_{3}$, though the localization length of the electrons is not large enough to be a macroscopic scale over the whole sample, it still exceeds the nearest metal-metal distance $(\sim 3.8 \AA)$ according to the conventional variable-range hopping. Thus, it is possible that, within the localization range, the magnetic $\mathrm{Co}^{3+}$ ions are FM coupled via the itinerant electron provided by $\mathrm{Ni}^{3+}$.

In fact, the carrier induced ferromagnetism in the insulating state has been found in Mn-based dilute magnetic semiconductors (DMS). For the representative compounds, III-V (Ga, Mn)As, II-VI (Zn, Mn)Te and IV-VI (Pb, Mn)Te [81, 53, 30, 19], the parent compound is a nonmagnetic insulator, while the doped holes induce a weak ferromagnetic coupling between $\mathrm{Mn}^{2+}$ ions. The nature of the magnetic interaction was found to fit into the framework of a RKKY-like mechanism based on the 
comparison of the experimental data with the theoretical calculations [53, 39].

Finally, there are two details worth being considered. First, regarding the 1.1 meV excitation, one may also expect to observe the excitations corresponding to the transitions of higher excited levels, such as $|1\rangle \rightarrow|2\rangle$ at $\sim 2.3 \mathrm{meV}$, but at base temperature the already very weak intensity of the $1.1 \mathrm{meV}$ excitation indicates a scarce population of the ground state, which makes the observation of the $|1\rangle \rightarrow|2\rangle$ transition practically impossible. However, as the temperature rises, the population of the excited states increase, and this should increase the chance of observing the excitation at $\sim 2.3 \mathrm{meV}$ and even more excitations at higher energies, but unfortunately warming also causes the $0.6 \mathrm{meV}$ excitation and the quasi-elastic scattering, which obviously dominate the spectra (Fig. 6.11 and 6.14). In our attempts of scanning the energy spectra up to $10 \mathrm{meV}$ and temperature to $50 \mathrm{~K}$, no other excitations have been resolved.

Second, the charge carrier of $\mathrm{LaCo}_{1-y} \mathrm{Ni}_{y} \mathrm{O}_{3}$ may have electron as well as hole character because the sign of the thermoelectric power measurements on $5 \%$ and $10 \%$ doped compounds was found to be positive [41]. The positive sign was also observed in other electron doped perovskite cobaltites $\mathrm{LaCo}_{1-y} \mathrm{Ti}_{y} \mathrm{O}_{3}$ and $\mathrm{DyCo}_{1-y} \mathrm{Ti}_{y} \mathrm{O}_{3}$ [37] ( $\mathrm{Ti}$ is $4+$, and to balance the charge, part of the Co ions are at $2+$, i.e. $\left.\mathrm{LaCo}_{1-2 y}^{3+} \mathrm{Co}_{y}^{2+} \mathrm{Ti}_{y}^{4+} \mathrm{O}_{3}\right)$. The thermoelectric power depends on a factor, $n_{h} \mu_{h}+n_{e} \mu e$, where $n_{h}$ and $n_{e}$ are the hole and electron density and $\mu_{h}$ and $\mu_{e}$ are the hole and electron mobilities. Thus, though Ni doping nominally introduces electrons, the presence of a small amount of 
holes with a much higher mobility could dominate the charge carrier character, and the magnetic $\mathrm{Co}^{3+}$ ion certainly creates $t_{2 g}$ holes regardless of being in the IS or HS state.

\subsection{Summary}

Based on our bulk magnetization and resistivity measurements and the neutron scattering experiments, three fundamental issues concerning the isovalent magnetic ion doped pervoskite cobaltite, $\mathrm{LaCo}_{1-y} \mathrm{Ni}_{y} \mathrm{O}_{3}$ and $\mathrm{LaCo}_{1-y} \mathrm{Fe}_{y} \mathrm{O}_{3}$, are discussed:

(1) The ground state of $\mathrm{Co}^{3+}$ is magnetic in $\mathrm{LaCo}_{1-y} \mathrm{Ni}_{y} \mathrm{O}_{3}$, but it is the LS state in $\mathrm{LaCo}_{1-y} \mathrm{Fe}_{y} \mathrm{O}_{3}$.

(2) The magnetic correlations in $\mathrm{LaCO}_{1-y} \mathrm{Ni}_{y} \mathrm{O}_{3}$ are identified to be $\mathrm{FM}$ only throughout the whole phase diagram.

(3) Depending on the energy levels of $\mathrm{Co}^{3+}$ and $\mathrm{Ni}^{3+}$, the FM magnetic interaction can be via the super-exchange, $\mathrm{Ni}^{3+}-\mathrm{O}^{2-}-\mathrm{Co}^{3+}$, and the FM correlation can be confined in one or two dimensions; alternatively, the interaction can be a RKKY-like in nature, $\mathrm{Co}^{3+}-e^{-}-\mathrm{Co}^{3+}$, where the FM coupling occurs between the magnetic $\mathrm{Co}^{3+}$ ions and is mediated by the itinerant electrons provided by $\mathrm{Ni}^{3+}$. 


\section{Chapter 7}

\section{Spin Excitation in $\mathrm{PrCoO}_{3}$}

\subsection{Introduction}

For the other members of rare-earth perovskite cobaltites, $\mathrm{RCoO}_{3}\left(\mathrm{R}^{3+}=\mathrm{Pr}^{3+}\right.$, $\left.\mathrm{Nd}^{3+} \ldots\right)$, the size of the A-site rare earth ion steadily decreases with increasing atomic number from lanthanum (atomic number 57) through lutetium (atomic number 71) due to lanthanoid contraction. This further enlarges the mismatch between the A-O and B-O bonds for the structure to be the ideal cubic symmetry. In $\mathrm{LaCoO}_{3}$ the lattice adjusts to the mismatch by adopting the trigonal symmetry $\mathrm{R} \overline{3} \mathrm{c}$ and in the other compounds, $\mathrm{RCoO}_{3}\left(\mathrm{R}^{3+}=\mathrm{Pr}^{3+}, \mathrm{Nd}^{3+} \ldots\right)$, the lattice adopts an even lower symmetry, orthorhombic Pnma [83, 40]. Fig. 7.1 shows how the oxygen octahedra change when the symmetry is lowered from cubic to orthorhombic: the Co-O-Co bonds are obviously buckled; and the Co-O bonds within one octahedron can have 


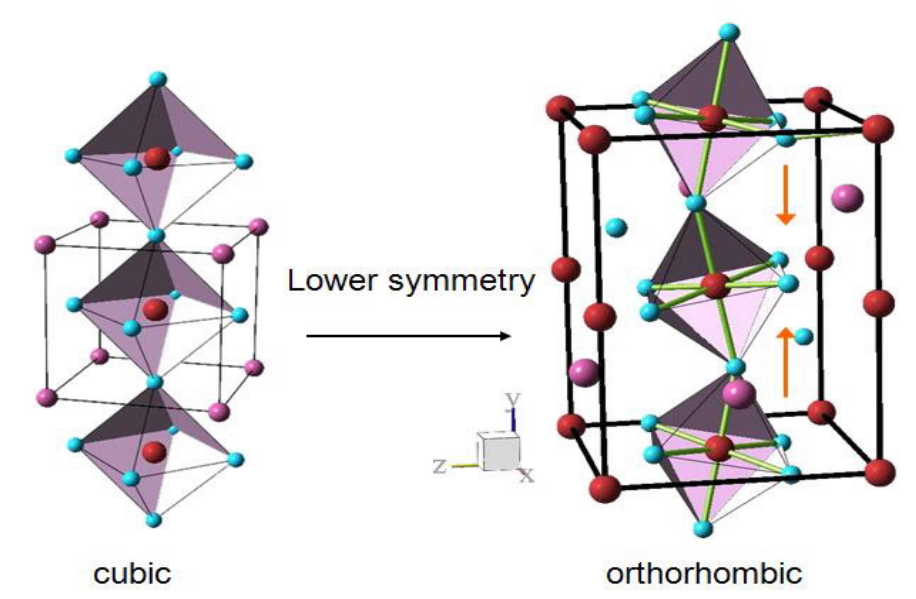

Figure 7.1: As the crystal symmetry lowers from cubic to orthorhombic, the octahedra become buckled to each other.

three different lengths.

Accompanying the change of structures it is found that the energy gap between the LS and the magnetic states increases from the order of $10^{2} \mathrm{~K}$ in $\mathrm{LaCoO}_{3}$ to the order of $10^{3} \mathrm{~K}$ in $\mathrm{EuCoO}_{3}[100,8]$. This is probably caused by the chemical pressure induced by the smaller A-site ion on the $\mathrm{CoO}_{6 / 2}$ octahedra, which increases the crystal field splitting and thus stabilizes Co ions at the LS state [6, 94].

Therefore, it is expected that the thermal spin-state-transition temperatures are raised higher in $\mathrm{RCoO}_{3}\left(\mathrm{R}^{3+}=\mathrm{Pr}^{3+}, \mathrm{Nd}^{3+} \ldots\right)$ than the one in $\mathrm{LaCoO}_{3}$. At the same time when temperature rises, $\mathrm{RCoO}_{3}$ will eventually become a metal. For example, 
based on the bulk resistivity and magnetization measurements in $\mathrm{LaCoO}_{3}$ the spinstate-transition occurs at $\sim 100 \mathrm{~K}$ and the IMT occurs at $\sim 500 \mathrm{~K}$. This temperature dependence was understood in terms of a transition from localized to itinerate behaviors of the $\mathrm{Co}^{3+}$ ions' $e_{g}$ electrons. In $\mathrm{RCoO}_{3}$, however, although the temperatures of the IMT are generally agreed upon, the temperatures of the spin-state transition and, consequently, the relation between the thermal spin excitations and the IMT are not clear. For example, based on the magnetic susceptibility and thermal conductivity on $\mathrm{LaCoO}_{3}, \mathrm{PrCoO}_{3}$ and $\mathrm{NdCoO}_{3}$, the temperatures at which the spin excitations begin are $35 \mathrm{~K}, 200 \mathrm{~K}$ and $300 \mathrm{~K}$, respectively [94]. Similar onset temperatures are also suggested by the temperature dependence of infrared spectroscopy, where anomalies were observed in $\mathrm{LaCoO}_{3}, \mathrm{PrCoO}_{3}$ and $\mathrm{NdCoO}_{3}$ around 120,220 and $275 \mathrm{~K}$ respectively, and these anomalies are suggested to be caused by an increase of new bands ascribed to the IS state of Co [82]. On the other hand, several experiments suggested the LS state was stabilized to higher temperatures until IMT; for example, ${ }^{59} \mathrm{Co} \mathrm{NMR}$ measurements indicated the transition in $\mathrm{PrCoO}_{3}$ occurred at least above $300 \mathrm{~K}$, and the spin-state transition and IMT happen at the same temperatures in $\mathrm{NdCoO}_{3}, \mathrm{SmCoO}_{3}$ and $\mathrm{EuCoO}_{3}$ at $\sim 600$ to $800 \mathrm{~K}[32,33]$.

In an effort to clarify this issue, the powder compound of $\mathrm{PrCoO}_{3}$ was studied from $\sim 10 \mathrm{~K}$ up to $350 \mathrm{~K}$ which is the temperature range below the $\mathrm{IMT}(\sim 600$ $\mathrm{K})$. The magnetism and the lattice properties were examined via the bulk magnetic susceptibility, neutron diffraction and inelastic scattering [98]. 


\subsection{When does Spin Excitation Occur in $\mathrm{PrCoO}_{3}$ ?}

\subsubsection{Bulk magnetization- Onset temperature $\sim 200 \mathrm{~K}$}

For $\mathrm{LaCoO}_{3}$, the thermal-induced spin-state excitation of $\mathrm{Co}^{3+}$ significantly increases the bulk magnetization from 30 to $100 \mathrm{~K}$. For the powder compound of $\mathrm{PrCoO}_{3}$ the bulk magnetic susceptibility was also measured as a function of temperature. However, for $\mathrm{PrCoO}_{3}$ both $\mathrm{Pr}^{3+}$ and $\mathrm{Co}^{3+}$ ions contribute to the magnetization. Fig. 7.2 shows the ZFC data for both $\mathrm{PrCoO}_{3}$ and $\mathrm{PrAlO}_{3}$. The $\mathrm{FC}$ data for both compounds are almost identical to the corresponding ZFC data, which indicates the magnetic moments of $\mathrm{Pr}^{3+}$ and $\mathrm{Co}^{3+}$ ions are not ordered. In this case, the contribution to the magnetization only from $\mathrm{Co}^{3+}$ ions in $\mathrm{PrCoO}_{3}$ is extracted by subtracting the data of $\mathrm{PrAlO}_{3}$ from that of $\mathrm{PrCoO}_{3}$ so that the magnetic signal from $\mathrm{Pr}^{3+}$ is excluded. This "effective" magnetization data is shown in Fig. 7.3, and the data of $\mathrm{LaCoO}_{3}$ is also plot to be compared. It can be seen that a minimum at $\sim 200 \mathrm{~K}$ appeared in the $\chi$ data of $\mathrm{PrCoO}_{3}$, and this is analogous to the minimum of $\sim 35 \mathrm{~K}$ in $\mathrm{LaCoO}_{3}$. Below these temperatures, $\chi(T)$ data showed Curie-Weiss-like behavior. For $\mathrm{LaCoO}_{3}$ this was suggested to be caused by the surface cobalt impurities. Above $200 \mathrm{~K}$ and $35 \mathrm{~K}$, the $\chi$ data of $\mathrm{PrCoO}_{3}$ and $\mathrm{LaCoO}_{3}$ start to increase, respectively. Therefore, the minimum in $\chi$ data suggests that the onset temperatures for the spin excitations are 35 and $200 \mathrm{~K}$ for $\mathrm{LaCoO}_{3}$ and $\mathrm{PrCoO}_{3}$, respectively. 


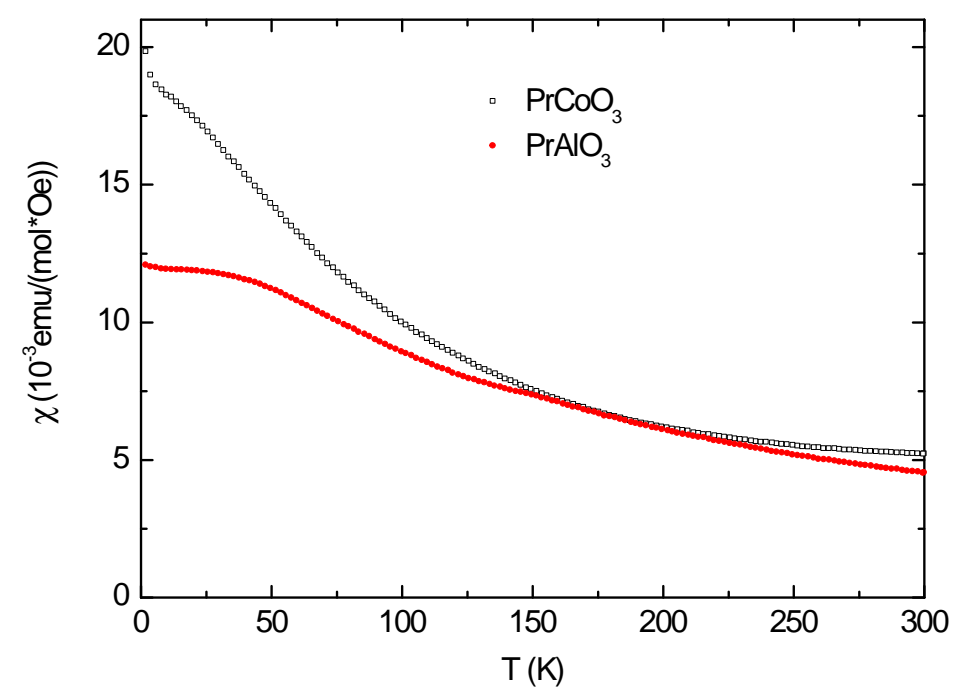

Figure 7.2: Temperature dependence of the bulk magnetic susceptibility of $\mathrm{PrCoO}_{3}$ and $\mathrm{PrAlO}_{3}$ under the applied field of $1000 \mathrm{Oe}$.

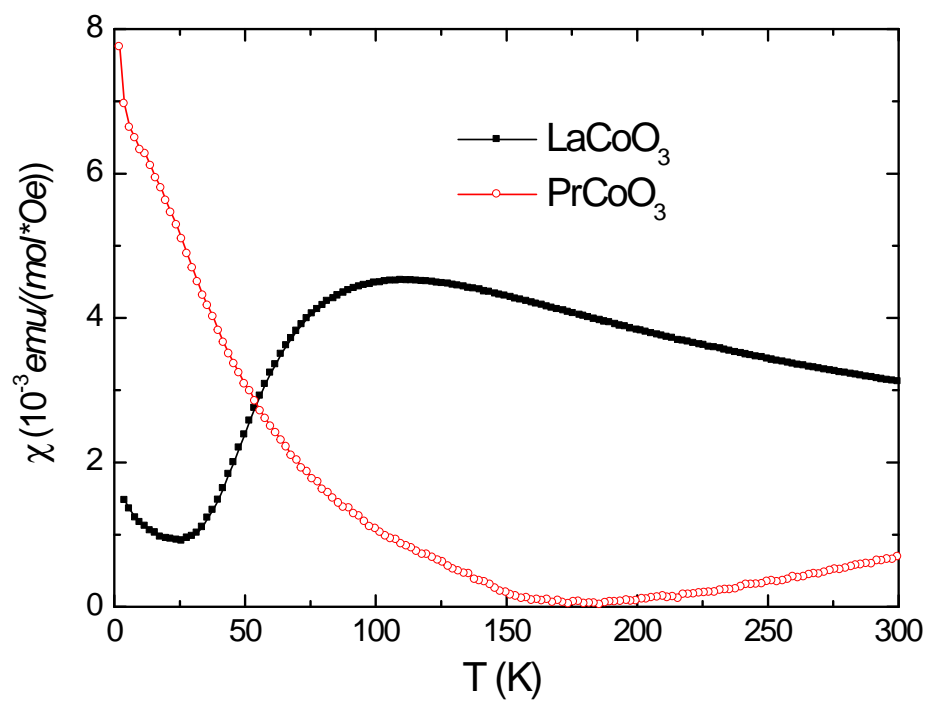

Figure 7.3: Temperature dependence of the bulk magnetic susceptibility of $\mathrm{PrCoO}_{3}$ after subtracting from $\mathrm{PrAlO}_{3}$. The data is compared with $\chi(T)$ of $\mathrm{LaCoO}_{3}$. 


\subsubsection{Crystal Structure- Absence of Distortion up to RT}

$\mathrm{Co}^{3+}$ ions in the IS state can cause Jahn-Teller distortions and the orthorhombic crystal symmetry is compatible with this distortion because the symmetry allows the Co-O bonds to be different lengths. Thus, an investigation of the crystal structure along with the local lattice would provide direct indication if the $\mathrm{IS}^{\mathrm{Co}^{3+}}$ ions exist. The neutron powder diffraction data were obtained on NPDF (Neutron Powder Diffractometer) at LANL from 12 to $350 \mathrm{~K}$. The Rietveld refinement indicated that the crystal structure in this temperature range is orthorhombic with space group Pnma (No. 62). Table 7.1 lists the refined lattice parameters, $a, b, c$ and the coordinations for $\operatorname{Pr}$ and two oxygen ions: the $\operatorname{Pr}$ position $(4 c)$ is at $\left(x_{\operatorname{Pr}}, \frac{1}{4}, z_{\operatorname{Pr}}\right)$, the oxygen ions are located at two different positions, one $(4 c)$ is at $\left(x_{O 1}, \frac{1}{4}, z_{O 1}\right)$ and the other $(8 d)$ is at $\left(x_{\mathrm{O} 2}, y_{\mathrm{O} 2}, z_{\mathrm{O} 2}\right)$. The Co position $(4 a)$ is at $(0,0,0)$ (As for the orthorhombic unit cell, please refer to Fig. 2.4).

Fig. 7.4 shows the Rietveld refinement results as a function of temperature from 12 to $350 \mathrm{~K}$. In (a) it can be seen that the lattice constants, $a, b$ and $c$ gradually increase with temperature. In (b) the unit cell volume is compared with the one of $\mathrm{LaCoO}_{3}$. An abrupt increase of the volume occurred in $\mathrm{LaCoO}_{3}$ around $100 \mathrm{~K}$, and it has been suggested to be caused by the increased population of excited $\mathrm{Co}^{3+}$ ion due to its larger ionic size than the one in the LS state. The unit cell volume of $\mathrm{PrCoO}_{3}$, however, does not show any anomalies, but increases at a slower rate, though as temperature rises to $350 \mathrm{~K}$ the slope becomes steeper. In accordance with 


\begin{tabular}{cccc}
\hline \hline $\mathrm{T}(\mathrm{K})$ & $a$ & $b$ & $c$ \\
\hline \hline 12 & $5.32992(3)$ & $7.55184(5)$ & $5.36577(3)$ \\
\hline 100 & $5.32998(3)$ & $7.55736(4)$ & $5.36546(2)$ \\
\hline 200 & $5.33175(3)$ & $7.56329(5)$ & $5.36892(3)$ \\
\hline 300 & $5.34112(4)$ & $7.57644(6)$ & $5.37738(4)$ \\
\hline 350 & $5.34744(4)$ & $7.58476(6)$ & $5.38260(4)$ \\
\hline \hline $\mathrm{T}(\mathrm{K})$ & $\operatorname{Pr}\left(x_{\operatorname{Pr}}, \frac{1}{4}, z_{\operatorname{Pr}}\right)$ & $\mathrm{O}_{1}\left(x_{O 1}, \frac{1}{4}, z_{O 1}\right)$ & $\mathrm{O}_{2}\left(x_{O 2}, y_{O 2}, z_{O 2}\right)$ \\
\hline \hline \multirow{3}{*}{12} & $0.46859(6)$ & $0.00631(6)$ & $0.28319(4)$ \\
& $-0.00491(9)$ & $0.06847(6)$ & $-0.03629(3)$ \\
& & & $0.21702(5)$ \\
100 & $0.46856(6)$ & $0.00624(7)$ & $0.28366(4)$ \\
& $-0.00479(11)$ & $0.06798(6)$ & $-0.03510(3)$ \\
& & $0.21650(5)$ \\
\multirow{3}{*}{200} & $0.46965(7)$ & $0.00586(8)$ & $0.28319(5)$ \\
& $-0.00473(13)$ & $0.06754(7)$ & $-0.03569(4)$ \\
& & & $0.21703(5)$ \\
300 & $0.47061(8)$ & $0.00601(9)$ & $0.28243(5)$ \\
& $-0.00452(15)$ & $0.06716(8)$ & $-0.03550(4)$ \\
& & & $0.21776(6)$ \\
\hline \multirow{3}{*}{350} & $0.47108(9)$ & $0.00610(10)$ & $0.28202(6)$ \\
& $-0.00462(17)$ & $0.06704(9)$ & $-0.03544(5)$ \\
& & & $0.21802(7)$ \\
\hline
\end{tabular}

Table 7.1: Rietveld Refinement Results for $\mathrm{PrCoO}_{3}$ from 12 to $350 \mathrm{~K}$. 

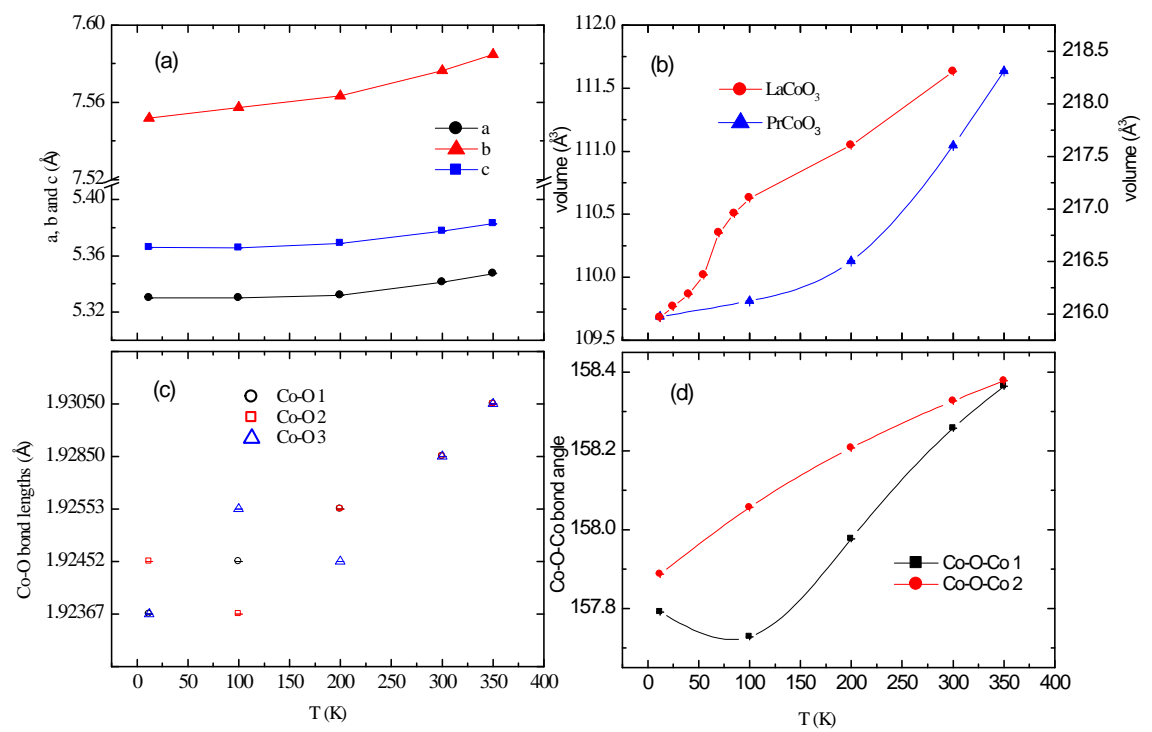

Figure 7.4: A summary of the Rietveld refinement results of $\mathrm{PrCoO}_{3}$ from 12 to 350 K. (a) the lattice parameters of orthorhombic unit cell, a, b and c. (b) the unit cell volume compared with the one of $\mathrm{LaCoO}_{3}$. (c) three $\mathrm{Co}-\mathrm{O}$ bond lengths in an octahedron. (4) two Co-O-Co bond angles.

the orthorhombic symmetry, there are three distinctive $\mathrm{Co}-\mathrm{O}$ bond lengths in one octahedron. However, the refinement results plotted in (c) show that the differences between the three Co-O bond lengths are as small as the order of $10^{-3} \AA$. The largest difference occurred at $100 \mathrm{~K}$, which also corresponds to the largest difference of two distinctive Co-O-Co bond angles, shown in (d). More importantly, as the temperature rises, the differences of Co-O bond lengths and Co-O-Co bond angles become less pronounced, which is opposite to the expectation assuming the excitation to the IS state occurred around $200 \mathrm{~K}$.

Our local structure study further confirmed the absence of the possible IS state $\mathrm{Co}^{3+}$ at least up to $350 \mathrm{~K}$. Fig. 7.5 is the $\mathrm{PDF}$ of $\mathrm{PrCoO}_{3}$ from $12 \mathrm{~K}$ to $350 \mathrm{~K}$. The 


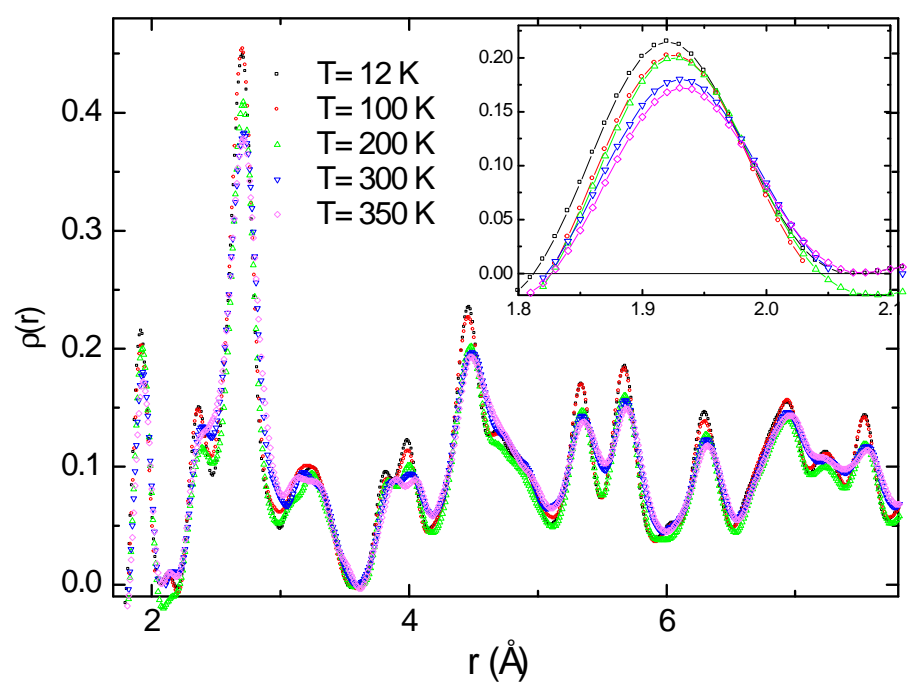

Figure 7.5: The $\mathrm{PDF}$ of $\mathrm{PrCoO}_{3}$ from 12 to $350 \mathrm{~K}$ obtained on the time-of-flight machine NPDF. The inset is the blow up of the first peak.

inset is an enlarged view of the first peak which corresponds to the Co-O bonds in the octahedron. Though the orthorhombic symmetry is compatible with the potential J-T distortion, there is no visible bond splitting in the first peak. Even the splitting purely due to the lattice symmetry is not visible due to the fine differences. As the temperature increases, all the peaks only became broader due to the increased thermal vibrations of the atoms around their equilibrium positions. Fig. 7.6 is a comparison of the experimental PDF at $12 \mathrm{~K}$ with the calculated model using Pnma symmetry, which appear to match each other very well.

In summary, there is no visible lattice distortion, at least up to $350 \mathrm{~K}$ based on both the Rietveld refinement and the PDF analysis. The absence of the lattice distortion with the space group of Pnma directly indicated the absence of IS $\mathrm{Co}^{3+}$. However, considering that $\mathrm{PrCoO}_{3}$ remains in the paramagnetic state, and the results based 


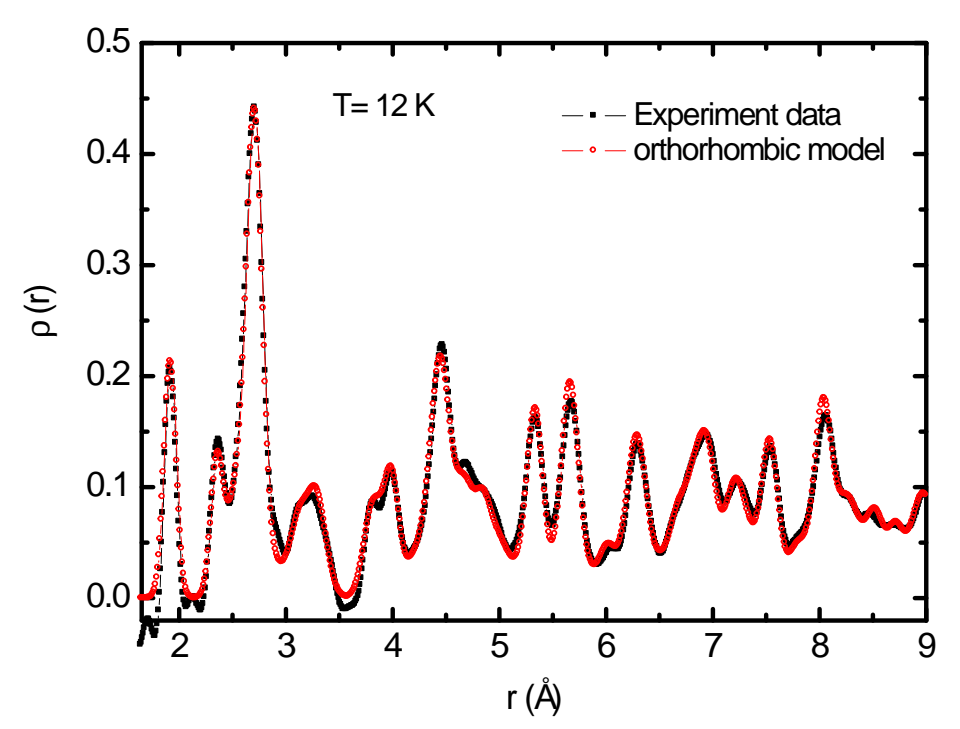

Figure 7.6: A comparison of the $\mathrm{PDF}$ of $\mathrm{PrCoO}_{3}$ at $12 \mathrm{~K}$ between the experiment data and the calculated model based on the orthorhombic crystal symmetry.

on the diffraction reflect an average information about the lattice, the absence of a static lattice distortion may not completely rule out the possibility for the occurrence of a spin-state transition which was suggested from the bulk magnetization. Thus, a preliminary search for a single ion excitation that is associated the thermal induced spin-state transition was carried out via inelastic neutron scattering.

\subsubsection{Low Energy Spectrum of $\mathrm{PrCoO}_{3}$}

The low energy spectra of $\mathrm{LaCoO}_{3}$ showed an excitation at $0.6 \mathrm{meV}$ with a clear temperature dependence, indicating a spin-flip excitation associated with the spinstate transition. For $\mathrm{PrCoO}_{3}$, based on the bulk $\chi$ which suggested onset temperature is $\sim 200 \mathrm{~K}$ a characteristic excitation was expected to appear starting from $\sim 200 \mathrm{~K}$. 
Thus, the energy scans on the powder sample of $\mathrm{PrCoO}_{3}$ were carried out on DCS from $10 \mathrm{~K}$ to $300 \mathrm{~K}$.

Fig. 7.7 shows the negative energy spectra at different temperatures. The spectra at all temperatures are clearly dominated by an excitation at $\sim 6 \mathrm{meV}$. Three other excitations at $\sim 7.5 \mathrm{meV}, \sim 10 \mathrm{meV}$ and $\sim 13.5 \mathrm{meV}$ are also recognized up to $150 \mathrm{~K}$. The $6 \mathrm{meV}$ excitation started to appear from the lowest temperature of $10 \mathrm{~K}$, and as temperature rose to $50 \mathrm{~K}$, its intensity significantly increased and the other three excitations started to show up. By $100 \mathrm{~K}$, the intensity of the $6 \mathrm{meV}$ excitation kept barely unchanged but became broadened and as temperature continued rising, the peak became much more broadened and the peak height started to be lowered.

In short, these excitations start from as low as $10 \mathrm{~K}$ and become stronger and broader as temperature rises. This is distinctively different from the temperature dependence of the excitation associated with the thermal spin-state transition, which needs to be "heated up" to a certain temperature. For $\mathrm{LaCoO}_{3}$, it takes $\sim 50 \mathrm{~K}$ for the excitation to be well recognized. For $\mathrm{PrCoO}_{3}$, as suggested by the bulk magnetic susceptibility measurements, the onset temperature of the possible thermal spin excitation is around $200 \mathrm{~K}$. Besides, the energy scale of these excitations is one order larger than the $0.6 \mathrm{meV}$ excitation. Therefore, the nature of these excitations is unlikely related to the thermal spin excitation. Instead, considering $\operatorname{Pr}^{3+}$ ion $\left(4 f^{1}\right)$ has one $f$ electron, the excitations are most likely associated with the crystal field splitting of the $f$ electron. The possible characteristic energy transfer associated with 


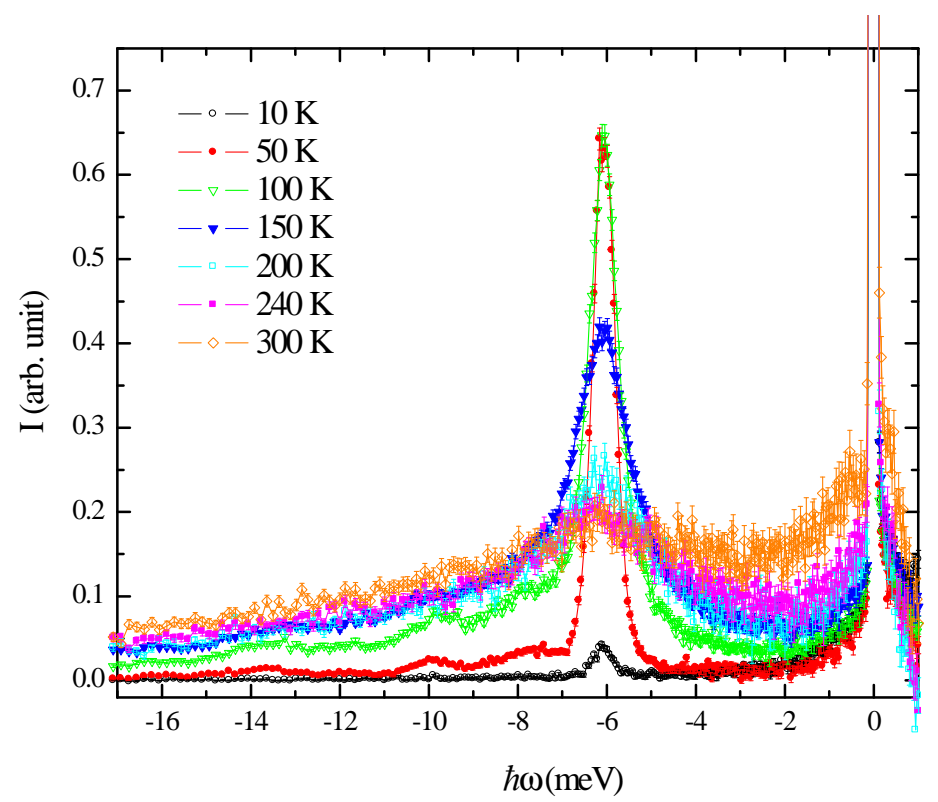

Figure 7.7: Temperature dependence of the energy spectra of $\mathrm{PrCoO}_{3}$ powder from 10 to $350 \mathrm{~K}$ obtained on DCS.

the spin excitation of $\mathrm{Co}^{3+}$ ion itself, however, has not been observed up to $350 \mathrm{~K}$.

\subsection{Summary}

For the other members of rare-earth perovskite cobaltites such as $\mathrm{PrCoO}_{3}$, the smaller size of A-site ions than $\mathrm{La}^{3+}$ results in the lower lattice symmetry, orthorhombic Pnma, which may raise the crystal field splitting and, therefore, the onset temperature for the thermally induced spin-state excitation. The bulk magnetic susceptibility measurements suggested that the spin excitation starts from around $200 \mathrm{~K}$. However, no lattice distortion was observed up to $350 \mathrm{~K}$ based on the neutron diffraction data via Rietveld refinement and PDF analysis, indicating the absence of static 
IS $\mathrm{Co}^{3+}$. The inelastic neutron experiment was then followed up to measure the low energy spectra up to $16 \mathrm{meV}$ as a function of temperature. However, the spectra were dominated by the excitations associated with the crystal field splitting of $f$ electrons of $\operatorname{Pr}^{3+}$ ions. The possible single-ion excitation analogous to the $0.6 \mathrm{meV}$ excitation in $\mathrm{LaCoO}_{3}$ may shift to above $350 \mathrm{~K}$ or may be covered by the strong inelastic intensities from $\mathrm{Pr}^{3+}$ ions. In the near future, in order to exclude these intensities the same energy spectra could by measured on $\mathrm{PrAlO}_{3}$ and then by subtracting them from the spectra of $\mathrm{PrCoO}_{3}$, the contribution only from $\mathrm{Co}^{3+}$ may be extracted. 


\section{Chapter 8}

\section{Conclusions}

The magnetism in doped $\mathrm{LaCoO}_{3}$ was studied via neutron scattering techniques. It was found that on an atomic scale, an unconventional magnetism in the insulating state of doped compounds is not uncommon in terms of the following: the spin states and the spin correlations are both correlated with a subtle lattice change due to doping whether it is A-site hole doping or B-site magnetic ion doping; On the other hand, the nature of magnetic interactions can be different depending on the dopants because it is determined by the interaction of magnetic $\mathrm{Co}^{3+}$ ions with holes or other magnetic ions such as $\mathrm{Ni}^{3+}$, which inherently is related with the electrical conductivity as a Mott insulator. In this dissertation, the effort towards gaining a microscopic understanding of the causes of unconventional magnetism and its consequences on the electric conductivity and the IMT was described. The following are several important results regarding the different doped systems. 
For the A-site hole doped systems, it had been previously known that coexistence of FM and IC magnetic phases prevails in the SG state of $\mathrm{La}_{1-x} \mathrm{Sr}_{x} \mathrm{CoO}_{3}$. The current work showed that such a magnetic phase separation is not unique to Sr doping. In $\mathrm{La}_{1-x} \mathrm{Ba}_{x} \mathrm{CoO}_{3}$, the IC correlation develops into an AFM order. With an increase in the $\mathrm{Ba}$ concentration, the AFM correlations eventually develop into long-range order. In $\mathrm{La}_{1-x} \mathrm{Ca}_{x} \mathrm{CoO}_{3}$, however, the AFM correlation is absent along with the SG state. The magnetic phase separations thus show a strong correlation with the A-site dopant. Considering that the ionic size of the divalent dopant increases significantly from $\mathrm{Ca}$ to $\mathrm{Sr}$ to $\mathrm{Ba}$ and that a resulting large A-site ionic size variance in $\mathrm{Sr}$ and Ba systems could possibly cause an inhomogenous local lattice, the magnetic phase separation and its trend may be understood to be driven by lattice inhomogeneity, where the subtle differences in the local lattice due to the dopants accommodates different magnetic correlations.

For the B-site magnetic ion doping, it was already known that the macroscopic magnetic and electric transport properties are essentially different in $\mathrm{LaCo}_{1-y} \mathrm{Ni}_{y} \mathrm{O}_{3}$ and $\mathrm{LaCo}_{1-y} \mathrm{Fe}_{y} \mathrm{O}_{3}$. The difference suggested that the ground state of $\mathrm{Co}^{3+}$ ions could be magnetic in $\mathrm{LaCo}_{1-y} \mathrm{Ni}_{y} \mathrm{O}_{3}$ but nonmagnetic in $\mathrm{LaCo}_{1-y} \mathrm{Fe}_{y} \mathrm{O}_{3}$. In the current work, this was confirmed through microscopic evidence of a lattice distortion and a magnetic excitation which are associated with $\mathrm{Co}^{3+}$ in the magnetic states. It was found that while the lattice of both systems has a trigonal symmetry, an enhanced lattice distortion along the trigonal axis was only observed in $\mathrm{LaCo}_{1-y} \mathrm{Ni}_{y} \mathrm{O}_{3}$. It was 
argued that this distortion is invoked to break the degeneracy of the magnetic $\mathrm{Co}^{3+}$ ions while maintaining the $\mathrm{Co}-\mathrm{O}$ bonds at a constant length. A magnetic excitation at $\sim 1.1 \mathrm{meV}$ at $\mathrm{T} \rightarrow 0 \mathrm{~K}$ was identified only in lightly doped $\mathrm{LaCo}_{1-y} \mathrm{Ni}_{y} \mathrm{O}_{3}$. The intensity of this excitation cannot be fully accounted by the trace amount of $\mathrm{Ni}^{3+}$ ions, and thus some of the $\mathrm{Co}^{3+}$ ions bear non-zero spins even without warming.

Furthermore, for $\mathrm{LaCo}_{1-y} \mathrm{Ni}_{y} \mathrm{O}_{3}$ it was previously reported that on its insulating side the resistivity follows variable range hopping and its IMT was characterized to be Mott-Anderson type which occurred at a much higher doping concentration than the FM transition. On the other hand, it was known that in the hole doped systems, the IMT was coincident with a FM transition, and the IMT was understood to be caused by a percolation of FM metallic clusters in the frame of DE. This suggested that the role of magnetism upon the IMT could be fundamentally different between hole doping and $\mathrm{Ni}$ doping; the magnetic exchange mechanism in $\mathrm{LaCo}_{1-y} \mathrm{Ni}_{y} \mathrm{O}_{3}$ is therefore insufficiently described by DE. However, little was known about $\mathrm{LaCo}_{1-y} \mathrm{Ni}_{y} \mathrm{O}_{3}$. In the current work, this issue was addressed through investigations of the microscopic magnetism of $\mathrm{LaCo}_{1-y} \mathrm{Ni}_{y} \mathrm{O}_{3}$ from the paramagnetic state to the $\mathrm{SG}$ state. It was found that the magnetic correlation in $\mathrm{LaCo}_{1-y} \mathrm{Ni}_{y} \mathrm{O}_{3}$ was exclusively $\mathrm{FM}$ and that when it is in the paramagnetic state, the FM spin fluctuations were associated with a $1.1 \mathrm{meV}$ excitation. These results suggested that the interaction of the spins of $\mathrm{Co}^{3+}$ with the doped electrons is fundamentally different from the interaction with holes. This has direct consequences on the magnetism and its relation with the electron 
conductance and IMT. Two possible scenarios are proposed: (1) the FM coupling can be a FM super-exchange via $\mathrm{Ni}^{3+}-\mathrm{O}^{2-}-\mathrm{Co}^{3+}$ and because the dopant $\mathrm{Ni}^{3+}$ brings about an electron in an $e_{g}$ orbital unlike hole doping which provides two empty $e_{g}$ orbitals, the FM couplings can be constrained into one- and/or two- dimensional configurations, which have a lower chance to percolate. (2) The nature of FM coupling is fundamentally different from super-exchange if the $1.1 \mathrm{meV}$ excitation is associated with the excitation energy levels for FM coupled spins which are not necessary the closest neighbors, because the extracted magnetic exchange constant is one order smaller than DE. A RKKY-like mechanism within the immediate vicinity of dopant $\mathrm{Ni}^{3+}$ where the FM coupling is mediated by the itinerant electrons of $\mathrm{Ni}^{3+}$ via $\mathrm{Co}^{3+}$ $e^{-}-\mathrm{Co}^{3+}$ is therefore proposed.

Finally, it has already been known that the temperature for the thermal spin-state transition is raised higher in other cobaltites, such as $\mathrm{PrCoO}_{3}$. However, it has not been clear exactly what temperature the spin excitation occurs as well as the relation between the spin excitation and IMT. In the current work, the neutron scattering diffraction and spectrum suggested that the excitation may occur at a temperature that is at least above $300 \mathrm{~K}$.

In summary, the current work provides a significant amount of information about magnetism on an atomic scale in a series of perovskite cobaltites. The interplay between the lattice, magnetism and conductivity was thoroughly discussed. 


\section{Bibliography}

[1] A. Abragam and B. Bleaney, Electron Paramagnetic Resonance of Transition Ions, Clarendon Press, Oxford, 1970.

[2] P. W. Anderson and H. Hasesawa, Phys. Rev. 100, 675 (1955).

[3] J. Androulakis, N. Katsarakis, Z. Viskadourakis, and J. Giapintzakis, J. Appl. Phys. 93, 5484 (2003).

[4] K. Asai, O. Yokokura, N. Nishimori, H. Chou, J. M. Tranquada, G. Shirance, S. Higuchi, Y. Okajima and K. Kohn, Phys. Rev. B 50, 3025 (1994).

[5] K. Asai, A. Yoneda, O. Yokokura, J. M. Tranquada, G. Shirane, and K. Kohn, J. Phys. Soc. Jpn. 67, 290 (1998).

[6] J. Baier, S. Jodlauk, M. Kriener, A. Reichl, C. Zobel, H. Kierspel, A. Freimuth, and T. Lorenz, Phys. Rev. B 71, 014443 (2005).

[7] J. G. Bednorz and K. A. Mueller, Zeitschrift für Physik B 64 (2), 189 (1986). 
[8] K. Berggold, M. Kriener, P. Becker, M. Benomare, M. Reuther, C. Zobel, and T. Lorentz, Phys. Rev. B 78, 134402 (2008).

[9] I. B. Bersuker, Electronic Structure and Properties of Transition Metal Compounds: Introduction to the Theory, John Wiley and Sons, Incorporated, New York (1996).

[10] V. G. Bhide, D. S. Rajoria, G. R. Rao, and C. N. R. Rao, Phys. Rev. B 6, 1021 (1972).

[11] G. Blasse, J. Appl. Phys. 36, 879 (1965).

[12] S. Blundell, Magnetism in Condensed Matter, Oxford University Press (2001).

[13] J. C. Burley, J. F. Mitchell, and S. Short, Phys. Rev. B 69, 054401 (2004).

[14] R. Caciuffo, D. Rinaldi, G. Garucca, J. Mira, J. Rivas, M. A. Senaris-Rodriguez, P. G. Radaelli, D. Fiorani, and J. B. Goodenough, Phys. Rev. B 59, 1068 (1999).

[15] F. A. Cotton, Chemical Applications of Group Theory, John Wiley \& Sons, New York 1990.

[16] P. -G. de Gennes, Phys. Rev. 118, 141 (1960).

[17] T. Egami and S. Billinge, Underneath the Bragg Peak: Structural Analysis of Complex Materials, Pergamon Press, Oxford (2003).

[18] This figure comes from http://mooni.fccj.org/ ethall/cobalt/cobalt.htm 
[19] D. Ferrand, J. Cibert, A. Wasiela, C. Bourgognon, S. Tatarenko, and G. Fishman, T. Andrearczyk, J. Jaroszynski, S. Kolesnik, and T. Diet, B. Barbara and D. Dufeu, Phys. Rev. B 63, 085201 (2001).

[20] J. Fontcuberta, B. Martı'nez, A. Seffar, S. Piñol, J. L. Garcı'a-Muñoz, and X. Obradors, Phys. Rev. Lett. 76, 7 (1996).

[21] Y. Fujikawa, K. Nakajima and T. Sakurai, Frontiers in Materials Research, Springer (2008).

[22] The schematic of image furnace comes from the web page: http://www2.warwick.ac.uk/fac/sci/physics/research/ condensedmatt/supermag/research/crystal_growth/

[23] A. Furrer and H. U. Gudel, J. Mag. Mag. Mat. 14, 256 (1979).

[24] V. Gnezdilov, V. Fomin, A. V. Yeremenko, K. Y. Choi, Y. Pashkevich, P. Lemmens, S. Shiryaev, G. Bychkov, and S. Barilo, Low Temp. Phys. 32, 162 (2006).

[25] J. B. Goodenough, Phys. Rev. 164, 2 (1967).

[26] J. B. Goodenough, J. App. Phys. 37, 3 (1966).

[27] J. B. Goodenough and J. -S. Zhou, Chem. Mater. 10, 2980 (1998). 
[28] J. B. Goodenough, Localized to Itinerant Electronic Transition in Perovskite Oxides, Springer, Berlin (2001).

[29] D. Hammer, J. Wu and C. Leighton, Phys. Rev. B 69, 134407 (2004).

[30] A. Haury, A. Wasiela, A. Arnoult, J. Cibert, S. Tatarenko, T. Dietl, and Y. Merle d'Aubigne, Phys. Rev. Lett. 79, 511 (1997).

[31] R. R. Heikes, R. C. Miller and R. Mazelsky, Physica 30, 1600 (1964).

[32] M. Itoh, M. Mori, S. Yamaguchi, and Y. Tokura, Physica B 259-261, 902 (1999).

[33] M. Itoh, J. Hashimoto, S. Yamaguchi, and Y. Tokura, Physica B 281-282, 510 (2000).

[34] M. Itoh, I. Natori, S. Kubota, and K. Motoya, J. Phys. Soc. Jpn. 63, 1486 (1994).

[35] A. Ishikawa, J. Nohara, and S. Sugai, Phys. Rev. Lett. 93, 136401 (2004).

[36] H. A. Jahn and E. Teller, Proc. Roy. Soc. A161, 220 (1937).

[37] Z. Jirak, J. Hejtmanek, K. Knizek, and M. Veverka, Phys. Rev. B 78, 014432 (2008).

[38] D. V. Karpinsky, I. O. Troyanchuk, K. Barner, H. Szymczak, and M. Tovar, J. Phys.: Condens. Matter 17, 7219 (2006). 
[39] H. Kepa, Le Van Khoi, C. M. Brown, M. Sawicki, J. K. Furdyna, T. M. Giebultowicz, and T. Dietl, Phys. Rev. Lett. 91, 087205 (2003).

[40] K. Knizek, J. Hejtmanek, Z. Jirak, P. Tomes, P. Henry and G. Andre, Phys. Rev. B 79, 134103 (2009).

[41] Y. Kobayashi, S. Murata, K. Asai, J. M. Tranquada, G. Shirane and K. Kohn, J. Phys. Soc. Jpn. 68, 1011 (1999).

[42] W. C. Koehler and E. O. Wollan, J. Phys. Chem. Solids 2, 100 (1957).

[43] M. A. Korotin, S. Yu. Ezhov, I. V. Solovyev, V. I. Anisimov, D. I. Khomskii and G. A. Sawatzky, Phys. Rev. B 54, 5309 (1996).

[44] M. Kriener, C. Zobel, A. Reichl, J. Baier, M. Cwik, K. Berggold, H. Kierspel, O. Zabara, A. Freimuth, and T. Lorenz, Phys. Rev. B 69, 094417 (2004).

[45] T. Kyomen, R. Yamazaki, and M. Itoh, Phys. Rev. B 68, 104416 (2003).

[46] S. -H. Lee's Ph.D thesis, Appendix A.

[47] S. -H. Lee, C. Broholm, W. Ratcliff, G. Gasparovic, Q. Huang, T. H. Kim and S. -W. Cheong, Nature 418, 856 (2002).

[48] P. Littlewood, Nature (London) 399, 529 (1999).

[49] D. Louca, J. L. Sarrao, J. D. Thompson, H. Roder, and G. H. Kwei, Phys. Rev. $B$ 60, 10378 (1999). 
[50] D. Louca and J. L. Sarrao, Phys. Rev. Lett 91, 155501(4) (2003).

[51] S. W. Lovesey, Theory of Neutron Scattering from Condensed Matter (Clarendon Press, Oxford, 1984).

[52] G. Maris, Y. Ren, V. Volotchaev, C. Zobel, T. Lorenz, and T. T. M. Palstra, Phys. Rev. B 67, 224423 (2003).

[53] F. Matsukura, H. Ohno, A. Shen, and Y. Sugawara, Phys. Rev. B 57, R2037 (1998).

[54] M. L. Medarde, J. Phys.: Condens. Matter 9, 1679 (1997).

[55] H. D. Megaw and C. N. W. Darlington, Acta Cryst. A 31, 161 (1975).

[56] N. F. Mott and R. Peierls, Proceedings of the Physical Society of London 49 (4S), $72(1937)$.

[57] N. F. Mott, Proceedings of the Physical Society of London A62 (7), 416 (1949).

[58] S. Noguchi, S. Kawamata, K. Okuda, H. Nojiri, and M. Motokawa, Phys. Rev. $B$ 66, 094404 (2002).

[59] Pradyot Patnaik, Handbook of Inorganic Chemical Compounds (McGraw-Hill Professional, 2002).

[60] J. Perez, J. Garcia, J. Blasco, and J. Stankiewicz, Phys. Rev. B 80, 2401 (1998). 
[61] D. Phelan, Despina Louca, S. Rosenkranz, S.-H. Lee, Y. Qiu, P. J. Chupas, R. Osborn, H. Zheng, J. F. Mitchell, J. R. D. Copley, J. L. Sarrao, and Y. Moritomo, Phys. Rev. Lett. 96, 027201 (2006).

[62] D. Phelan, D. Louca, K. Kamazawa, S. H. Lee, S. N. Ancona, S. Rosenkranz, Y. Motome, M. F. Hundley, J. F. Mitchell and Y. Moritomo, Phys. Rev. Lett. 97, 235501 (2006).

[63] D. Phelan, D. Louca, K. Kamazawa, M. F. Hundley, and K. Yamada, Phys. Rev. B 76, 104111 (2007).

[64] D. Phelan, J. Yu and Despina Louca, Phys. Rev. B 78, 094108 (2008).

[65] V. P. Plakhty, P. J. Brown, B. Grenier, S. V. Shiyaev, S. N. Barilo, S. V. Gavrilov, and E. Ressouhe, J. Phys: Cond. Matt. 18, 3517 (2006).

[66] A. Podlesnyak, S. Streule, J. Mesot, M. Medarde, E. Pomjakushina, K. Conder, A. Tanaka, M. W. Haverkort, and D. I. Khomskii, Phys. Rev. Lett. 97, 247208 (2006).

[67] A. Podlesnyak, M. Russina, A. Furrer, A. Alfonsov, E. Vavilova, V. Kataev, B. Buchner, Th. Strassle, E. Pomjakushina, K. Conder, and D. I. Khomskii, Phys. Rev. Lett. 101, 247603 (2008).

[68] P. M. Raccah and J. B. Goodenough, Phys. Rev. 155, 932 (1967).

[69] P. G. Radaelli and S. W. Cheong, Phys. Rev. B 66, 094408 (2002). 
[70] H. M. Rietveld, J. App. Cryst. 2, 65 (1969).

[71] Z. Ropka and R. J. Radwanski, Phys. Rev. B 67, 172401 (2003).

[72] Z. Ropka and R. J. Radwanski, Physica B 312, 777 (2002).

[73] S. Rosenkranz and D. Phelan, unpublished data.

[74] The $\mathbf{t}_{\text {average }}$ is deduced from the data reported by V G Sathey, A V Pimpaley, V Siruguriz and S K Paranjpex, J. Phys.: Condens. Matter 8 3889-3896 (1996).

[75] P. Schiffer, A. P. Ramirez, W. Bao, and S.-W. Cheong, Phys. Rev. Lett. 75, 3336 (1995).

[76] M. A. Senaris-Rodriguez and J. B. Goodenough, J. Solid State Chem. 118, 323 (1995).

[77] R. D. Shannon, Acta Cryst. A 32, 751 (1976).

[78] G. Shirane, S. M. Shapiro, and J. M. Tranquada, Neutron Scattering with a Triple-Axis Spectrometer, Cambridge University Press, Cambridge (2002).

[79] J. Singleton, Band Theory and Electronic Properties of Solids, Oxford University Press (2001).

[80] G. L. Squires, Introduction to the Theory of Thermal Neuntron Scattering (Cambridge University Press, Oxford, 1978). 
[81] T. Story, R. R. Galazka, R. B. Frankel and P. A. Wolff, Phys. Rev. Lett. 56, 777 (1986).

[82] L. Sudheendra, M. M. Seikh, A. R. Raju, C. Narayana, Chem. Phys. Lett. 340, $275(2001)$.

[83] T. Takami, J. -S. Zhou, J. B. Goodenough, and H. Ikuta, Phys. Rev. B 76, $144116(2007)$.

[84] G. Thorton, F. C. Morrison, S. Partington, B. C. Tofield, and D. E. Williams, J. Phys. C 21, 2871 (1988).

[85] G. Thornton, B. C. Tofield, and A. W. Hewat, J. Solid State Chem. 61, 301 (1986).

[86] Y. Tokura and N. Nagaosa, Science 288, 462 (2000).

[87] I. O. Troyanchuk, D.V. Karpinskii, V. M. Dobryanskii, Yu. A. Fedotova, and H. Szymczak, J. Exp. and Theo. Phy., 100, 1121 (2005).

[88] M. Uehara, S. Mori, C. H. Chen, and S.-W. Cheong, Nature (London) 399, 560 (1999).

[89] J. Van Vleck, Phys. Rev. 41, 208 (1932).

[90] J. Wu and C. Leighton, Phys. Rev. B 67, 174408 (2003). 
[91] S. Yamaguchi, Y. Okimoto, H. Taniguchi, and Y. Tokura, Phys. Rev. B 53, R2926 (1996).

[92] S. Yamaguchi, H. Taniguchi, H. Takagi, T. Arima and Y. Tokura, J. Phys. Soc. Jpn. 64, 1885 (1995).

[93] J. -Q. Yan, J. -S. Zhou, and J. B. Goodenough, Phys. Rev. B 70, 014402 (2004).

[94] J. -Q. Yan, J. -S. Zhou and J. B. Goodenough, Phys. Rev. B 69, 134409 (2004)

[95] J. Yu, D. Louca, D. Phelan, K. Tomiyasu, K. Horigane, and K. Yamada, Phys. Rev. B 80, 052402 (2009).

[96] J. Yu, K. Kamazawa, and D. Louca, Phys. Rev. B 82, 224101 (2010).

[97] J. Yu, D. Phelan, and D. Louca, in preparation.

[98] J. Yu and D. Louca, unpublished data.

[99] C. Zener, Phys. Rev. 82, 403 (1951).

[100] J. -S. Zhou, J. -Q. Yan, and J. B. Goodenough, Phys. Rev. B 71, 220103(R) (2005).

[101] C. Zobel, M. Kriener, D. Bruns, J. Baier, M. Gruninger, T. Lorenz, P. Reutler, and A. Revcolevschi, Phys. Rev. B 66, 020402(R) (2002). 